\title{
DISSIPATIVE OPERATORS AND HYPERBOLIC SYSTEMS OF PARTIAL DIFFERENTIAL EQUATIONS
}

\author{
BY \\ R. S. PHILLIPS( $\left.{ }^{1}\right)$
}

1. Introduction. The original purpose of this research was to employ the theory of semi-groups of operators to obtain a solution of the Cauchy problem for dissipative hyperbolic systems of partial differential equations with time invariant coefficients and boundary conditions. Here the term "dissipative" is used to emphasize the basic assumption imposed on the system, namely, that the energy is nonincreasing in time. As the work progressed, it became clear that the argument could be given an abstract formulation and that the results on hyperbolic systems should be treated as an application of the general theory of dissipative operators $\left({ }^{2}\right)$.

Definition 1.1. Let $H_{0}$ be a hilbert space with inner product $\left\langle y^{0}, z^{0}\right\rangle$. A linear operator $L$ with domain $\mathfrak{D}(L)$ is said to be dissipative if

$$
\left\langle L y^{0}, y^{0}\right\rangle+\left\langle y^{0}, L y^{0}\right\rangle \leqq 0,
$$$$
y^{0} \in \mathfrak{D}(L)
$$

and to be maximal dissipative if it is not the proper restriction of any other dissipative operator.

The connection between dissipative operators and dissipative hyperbolic systems is almost immediately evident. In fact, the dissipative assumption on the hyperbolic system suggests that a natural setting of the problem would be a hilbert space $H_{0}$ where $\left\|y^{0}\right\|^{2}=\left\langle y^{0}, y^{0}\right\rangle$ is a measure of the energy of the system at a given time. A semi-group solution to the Cauchy problem (see, for instance, E. Hille and R. S. Phillips [7]) would then consist of a oneparameter family of linear bounded operators $[S(t) ; t \geqq 0]$ such that

(i) $S\left(t_{1}+t_{2}\right)=S\left(t_{1}\right) S\left(t_{2}\right), t_{1}, t_{2}>0, S(0)=I$;

(ii) $S(t)$ is strongly continuous for $t \geqq 0$;

(iii) $\left\|S(t) y^{0}\right\|$ is nonincreasing in $t$ for each $y^{0} \in H_{0}$.

Condition (i) is a consequence of the initial-value problem being well set, (ii) results from the requirement $\lim _{t \rightarrow 0+} S(t) y^{0}=y^{0}$, and (iii) is a restatement of the dissipative assumption. Condition (iii) can also be expressed by saying that the $S(t)$ are contraction operators, that is, operators of norm $\leqq 1$. If $L$

Received by the editors April 19, 1957.

(1) This paper was written under the sponsorship of the Office of Naval Research, United States Navy, contract Nonr 228 (09).

(2) Dissipative operators are playing an increasingly important role as research on nonselfadjoint operators proceeds. Already, M. S. Livšic [9] and B. R. Mukminov [10] have obtained a remarkable expansion theorem for bounded dissipative operators having real parts of finite trace. 
is the infinitesimal generator, the differential system will be satisfied in the sense that

$$
\frac{d}{d t} S(t) y^{0}=L\left[S(t) y^{0}\right], \quad \text { for all } y^{0} \in \mathfrak{D}(L) \text { and } t>0,
$$

the derivative being taken in the norm topology of $H_{0}$. It turns out that an operator $L$ is the infinitesimal generator of a strongly continuous semi-group of contraction operators on $H_{0}$ if and only if $L$ is a maximal disipative operator with dense domain.

In searching for a maximal dissipative generator for our problem, one can start out with either a minimal operator $L_{0}$ or a maximal operator $L_{1}$, each representing the spatial part of the hyperbolic operator, and then determine the maximal dissipative extensions of $L_{0}$ and restrictions of $L_{1}$. These two procedures are duals of one another; the first can lead to operators which are no longer merely differential operators, whereas the second can result in differential operators whose domains need not be restricted by simple boundary conditions, but rather by "global" lateral conditions reminiscent of the lateral conditions found by W. Feller [5] in his treatise on parabolic partial differential equations. The maximal dissipative operators which are differential operators and whose domains are delimited by the usual type of boundary conditions are precisely those which are at the same time extensions of $L_{0}$ and restrictions of $L_{1}$. Chapter I is primarily concerned with giving an abstract characterization of all maximal dissipative operators $L$ such that $L_{0} \subset L \subset L_{1}$. In order to motivate our operator-theoretic development we shall now describe the hyperbolic system to which this theory will be applied in Chapter II.

Let $\Delta$ be a domain in the $m$-dimensional real euclidean space with points $\chi=\left(\chi^{1}, \chi^{2}, \cdots, \chi^{m}\right)$ and let $y^{0}(\chi, t)$ be a function of $(\chi, t)$ with values in a $k$-dimensional complex euclidean space. We consider the initial value problem

$$
\begin{aligned}
y_{t}^{0} & \left.=L_{1} y^{0} \equiv E^{-1}\left[\left(A^{i} y^{0}\right)_{i}+B y^{0}\right)\right], \quad \chi \in \Delta, t>0, \\
y^{0}(\chi, 0) & =f^{0}(\chi) ;
\end{aligned}
$$

here we use the tensor notation for summation, the subscript $i$ denoting differentiation with respect to $\chi^{i}$. The symbols $E, A^{i}$, and $B$ represent $k \times k$ matrix-valued functions of $\chi$ alone, $E$ being positive definite, and $A^{i}$ being hermitian, and the $A^{i}$ and $B$ together satisfying the "dissipative" condition

$$
B+B^{*}+A_{i}^{i} \leqq \Theta
$$$$
\chi \in \Delta,
$$

where $B^{*}$ is the adjoint of $B$ relative to the inner product

$$
\left(y^{0}, z^{0}\right)=\eta^{i \bar{\zeta}^{i}} .
$$

We further assume that in each compact subset of $\Delta$ the elements of $E$ are 
continuous, the elements of $A^{i}$ are absolutely continuous with respect to $\chi^{i}$, and the elements of $A_{i}^{i}$, and $B$ are square integrable; no restriction is placed on the growth properties of these functions near the boundary of $\Delta$. It will be convenient to set

$$
D \equiv E^{-1}\left[B+B^{*}+A_{i}^{i}\right] .
$$

If $y^{0}(\chi, t)$ is a solution of the system (1.2), then at a given time the energy of the associated physical model is given by

$$
\text { Energy }=\frac{1}{2} \int_{\Delta}\left(E y^{0}, y^{0}\right) d \chi .
$$

Using the energy as a measure we are thus lead to the hilbert space $H_{0}$ $=L_{2}(\Delta ; E)$ with inner product

$$
\left\langle y^{0}, z^{0}\right\rangle=\int_{\Delta}\left(E y^{0}, z^{0}\right) d \chi .
$$

After an integration by parts we obtain, at least formally, the following relation for the rate of change of energy from (1.2):

$$
\begin{aligned}
\left\langle y^{0}, y^{0}\right\rangle_{t} & =\left\langle L_{1} y^{0}, y^{0}\right\rangle+\left\langle y^{0}, L_{1} y^{0}\right\rangle \\
& =\int_{\Delta}\left(E D y^{0}, y^{0}\right) d \chi+\int_{\mathrm{\Gamma}}\left(A^{i} y^{0}, y^{0}\right) n^{i} d \sigma,
\end{aligned}
$$

where $n=\left(n^{1}, n^{2}, \cdots, n^{m}\right)$ is the outward normal to the boundary $\Gamma$ of $\Delta$ (supposing, for the moment, that $n$ exists). The volume integral on the right is the rate at which energy enters the system from interior sources, whereas the surface integral is the rate at which energy enters the system through the boundary. Since we shall require $\left\langle y^{0}, y^{0}\right\rangle_{t} \leqq 0$, in particular, for all smooth initial functions vanishing outside of compact subsets of $\Delta$, the condition (1.3) follows and has the physical significance of requiring that there be no internal energy sources.

Solutions to (1.2) whose boundary values satisfy the condition

$$
\int_{\Gamma}\left(A^{i} y^{0}, y^{0}\right) n^{i} d \sigma \leqq 0
$$

will clearly be dissipative. These are the solutions which are commonly associated with (1.2) and they are characterized by the fact that the corresponding generators lie between the previously defined minimal operator $L_{0}$ and maximal operator $L_{1}$; we shall call such solutions properly dissipative. On the other hand there are dissipative solutions of (1.2) which do not satisfy (1.8). For such solutions the energy entering through the boundary is offset by the energy losses due to internal sinks, and the domain of the corresponding gen- 
erator is delimited by global lateral conditions, as previously mentioned $\left({ }^{3}\right)$. Solutions of this kind are treated in the appendix to Chapter II.

In general, of course, the above boundary integral is not well defined. However, in situations where it is well defined, one sees that

$$
\int_{\Gamma}\left(A^{i} y^{0}, y^{0}\right) n^{i} d \sigma=\left\langle L_{1} y^{0}, y^{0}\right\rangle+\left\langle y^{0}, L_{1} y^{0}\right\rangle-\int_{\Delta}\left(E D y^{0}, y^{0}\right) d \chi .
$$

The relation (1.9) provides us with a method for defining the boundary integral whenever the terms on the right are meaningful and accordingly we take (1.9) as the definition of the boundary integral for an arbitrary domain $\Delta$. That the so-defined relation is actually a boundary functional can be seen from the fact that it vanishes for all continuously differentiable functions $y^{0}(\chi)$ with compact carriers in $\Delta\left({ }^{4}\right)$.

The presence of the term $\int_{\Delta}\left(E D y^{0}, y^{0}\right) d \chi$ in (1.9) gives rise to some difficulty since this integral will in general not exist for all of the members of $\mathfrak{I}\left(L_{1}\right)$ when this operator is suitably extended to be closed. We may avoid this difficulty by treating $L_{1}$ as a transformation on $H_{1}=L_{2}(\Delta ; E F)$ to $H_{2}$ $=L_{2}\left(\Delta ; E F^{-1}\right)$, where $F(\chi)$ is the matrix-valued function

(3) A simple example will serve to illustrate the kind of nonproperly dissipative extensions of $L_{0}$ which we have in mind. Let $y^{0}$ be a complex-valued function of $\chi, 0<\chi<1$, and set $L_{1} y^{0}=y_{x}^{0}-y^{0}, \mathfrak{D}\left(L_{1}\right)=\left[y^{0} ; y^{0}\right.$ absolutely continuous with $y^{0}$ and $y_{x}^{0}$ in $\left.L_{2}(0,1)\right]$. We further define $L_{0} \subset L_{1}$ by $\mathfrak{D}\left(L_{0}\right)=\left[y^{0} ; y^{0} \in \mathfrak{D}\left(L_{1}\right)\right.$ and $\left.y^{0}(0)=0=y^{0}(1)\right]$. It is clear that $L_{0}$ is dissipative, in fact, $\left\langle L_{1} y^{0}, y^{0}\right\rangle+\left\langle y^{0}, L_{1} y^{0}\right\rangle=-2\left\langle y^{0}, y^{0}\right\rangle+\left[\left|y^{0}(1)\right|^{2}-\left|y^{0}(0)\right|^{2}\right]$, which is obviously nonpositive for $y^{\circ} \in \mathfrak{D}\left(L_{0}\right)$. The second term in the right member represents the boundary integral (1.8) and one sees from this that a generator of properly dissipative solutions, say $L_{\alpha}$, must be a restriction of $L_{1}$ with domain $\mathfrak{D}\left(L_{\alpha}\right)=\left[y^{0} ; y^{0} \in \mathfrak{D}\left(L^{1}\right)\right.$ and $\left.y^{0}(1)=\alpha y^{0}(0)\right]$ for fixed $\alpha$ of absolute value $\leqq 1$.

On the other hand, for arbitrary $h^{0} \in L_{2}(0,1)$ with $\left\langle h^{0}, h^{0}\right\rangle \leqq 2$ the operator $L y^{0}=L_{1} y^{0}$ $+y^{0}(0) h^{0}, \mathfrak{D}(L)=\left[y^{0} ; y^{0} \in \mathfrak{D}\left(L_{1}\right)\right.$ and $\left.y^{0}(1)=0\right]$ is a maximal dissipative extension of $L_{0}$ (but not a restriction of $\left.L_{1}\right)$. In fact, it is clearly dissipative since $\left\langle L y^{0}, y^{0}\right\rangle+\left\langle y^{0}, L y^{0}\right\rangle=-\mid y^{0}(0)$ $-\left.\left\langle y^{0}, h^{0}\right\rangle\right|^{2}-\left[2\left\langle y^{0}, y^{0}\right\rangle-\left|\left\langle y^{0}, h^{0}\right\rangle\right|^{2}\right] \leqq 0$. In order to show that $L$ (or $L_{\alpha}$ ) is maximal dissipative it suffices to show, by the corollary to Theorem 1.1.1, that the range of $I-L$ (or of $I-L_{\alpha}$ ) is $L_{2}(0,1)$; and this can be accomplished by the usual green's function solution of $(I-L) y^{0}=f^{0}$. The system corresponding to $L$ requires a mechanism for feeding energy back into the interior with density $y^{0}(0) h^{0}$.

Since $L \supset L_{0}$, the adjoint of $L$, namely $M$, is a restriction of the adjoint of $L_{0}$ which we denote by $M_{1}$. It is readily seen that $M_{1} z^{0}=-z_{\chi}^{0}-z^{0}, \mathfrak{D}\left(M_{1}\right)=\left[z^{0} ; z^{0}\right.$ absolutely continuous with $z^{0}$ and $z_{x}^{0}$ in $\left.L_{2}(0,1)\right]$. Moreover $\left\langle L y^{0}, z^{0}\right\rangle-\left\langle y^{0}, M_{1} z^{0}\right\rangle=-y^{0}(0)\left[z^{0}(0)\right]-+y^{0}(0)\left\langle h^{0}, z^{0}\right\rangle$ and this vanishes for all $y^{0} \in \mathfrak{D}(L)$ (and hence for all $y^{0}(0)$ ) if and only if $z^{0}(0)=\left\langle z^{0}, h^{0}\right\rangle$. This condition determines the domain of $M C M_{1}$ and it follows from Theorem 1.1.2 that $M$ is a maximal dissipative restriction of $M_{1} . M$ is thus an example of a maximal dissipative restriction of a maximal operator $M_{1}$ with a domain delimited by global lateral conditions.

(4) If $y(\chi)$ vanishes outside of a compact subset of $\Delta$, one can replace $\Delta$ in the right member of (1.9) by a large cube $\Delta_{0}$ containing $\Delta$. If one then applies the Gauss theorem (see, for instance, $H$. Federer [4, pp. 313-315]), the right side of (1.9) becomes equal to the left side with $\Gamma$ now replaced by the boundary $\Gamma_{0}$ of $\Delta_{0}$, and it is clear that the integrand vanishes on $\Gamma_{0}$. 


$$
F=I-D \geqq I,
$$

In this case all of the volume integrals appearing in the right member of (1.9) exist even for the closure of the so-defined $L_{1}$.

With this in mind, we start with the transformation $L_{21}^{00}$ on $H_{1}$ to $H_{2}$ defined as

$$
L_{21}^{001} y^{1}=E^{-1}\left[\left(A^{i} y^{1}\right)_{i}+B y^{1}\right],
$$

$\mathfrak{D}\left(L_{21}^{00}\right)=\left[y^{1} ; y^{1}\right.$ continuously differentiable with compact carrier $]$,

and denote its strong closure by $L_{21}^{0}$ and its weak closure( $\left.{ }^{5}\right)$ by $L_{21}^{1}$. The previously mentioned operators $L_{0}$ and $L_{1}$ are the maximal restrictions of these respective transformations which have their ranges in $H_{0}$. We note that the so-defined $L_{1}$ is the operator of largest domain and of type (1.2) for which the boundary integral is meaningful in our extended sense. Consequently, any generator of properly dissipative solutions of (1.2) is necessarily a restriction of $L_{1}$. On the other hand $L_{0}$ is the largest restriction of $L_{1}$ whose domain consists only of functions which are essentially zero on the boundary. Thus the domain of an operator $L$ such that $L_{0} \subset L \subset L_{1}$ will be determined entirely by the boundary values assumed by its member functions. It turns out that each maximal dissipative operator of this type generates only properly dissipative solutions of (1.2) and, conversely, any generator of properly dissipative solutions of (1.2) is maximal dissipative and of this type.

Our problem, then, is to characterize all maximal dissipative operators $L$ such that $L_{0} \subset L \subset L_{1}$ and to this end we introduce the following.

Definition 1.2. Let $H_{12}=H_{1} \times H_{2}$ denote the product space of elements $y=\left[y^{1}, y^{2}\right]$ where $y^{1} \in H_{1}$ and $y^{2} \in H_{2}$ and set

$$
Q(y, z)=\left\langle y^{1}, z^{2}\right\rangle+\left\langle y^{2}, z^{1}\right\rangle-\int_{\Delta}\left(E D y^{1}, z^{1}\right) d x .
$$

$A$ linear subspace $N \subset H_{12}$ [or $P \subset H_{12}$ ] is said to be negative [positive] if

$$
Q(y, y) \leqq 0 \text { for all } y \in N \quad[Q(z, z) \geqq 0 \text { for all } z \in P] ;
$$

it is called maximal negative [maximal positive] if it is not the proper subspace of a negative [positive] subspace of $H_{12}$. The Q-orthogonal complement of a set $S$ is defined as

$$
[z ; Q(y, z)=0 \text { for all } y \in S] .
$$

Following J. W. Calkin [1] (cf. M. I. Višik [13] and Lars Hörmander

(5) More precisely, the operator $L_{21}^{1}$ is defined as follows. Set $M_{21}^{00} z^{1}=E^{-1}\left[-\left(A^{i} z^{1}\right)_{i}\right.$ $\left.+\left(B^{*}+A_{i}^{i}\right) z^{1}\right]$ with $\mathfrak{D}\left(M_{21}^{00}\right)=\mathfrak{D}\left(L_{21}^{00}\right)$ and let $\left(M_{21}^{00}\right) *$ denote its adjoint (on $H_{2}$ to $\left.H_{1}\right)$. Then $L_{21}^{1}=F^{-1}\left(M_{21}^{00}\right) * F$, where $F$ is the multiplicative operator taking $z^{1} \in H_{1}$ into $F(\cdot) z^{1}(\cdot) \in H_{2}$. 
[8]), we represent the boundary data assumed by functions in $\mathfrak{D}\left(L_{21}^{1}\right)$ by the cosets of the quotient space

$$
H=\leftrightarrow\left(L_{21}^{1}\right) / \leftrightarrow\left(L_{21}^{0}\right),
$$

where $\$\left(L_{21}^{i}\right)$ denotes the graph of $L_{21}^{i}, i=0,1$. As we shall see, the bilinear form $Q(y, z)$ depends only on the cosets to which $y$ and $z$ belong and hence can be used to define the form $Q\left(y^{0}, z^{0}\right)$ where $y^{0}, z^{0} \in H$ are the images of $y$ and $z$, respectively, under the natural homomorphism. Finally, there is a one-to-one correspondence between the maximal negative subspaces $N$ of $H$ (relative to $\mathrm{Q}$ ) and the maximal dissipative operators $L$ such that $L_{0} \subset L \subset L_{1}$; this correspondence is given by

$$
\mathfrak{D}(L)=\left[y^{0} ;\left[y^{0}, L_{1} y^{0}\right] \rightarrow N\right] .
$$

This, then, is the sense in which we have been able to characterize all properly dissipative solutions of (1.2).

We note that the formal adjoint of $L_{1}$ is of the form

$$
M_{1} z^{0}=E^{-1}\left[-\left(A^{i} z^{0}\right)_{i}+\left(B^{*}+A_{i}^{i}\right) z^{0}\right],
$$

and it is readily verified that

$$
z_{t}^{0}=M_{1} z^{0}
$$

is again a dissipative hyperbolic system. If we denote the adjoint of $L$ by $M$, then we find that $M$ is again maximal dissipative, $M_{0} \subset M \subset M_{1}$, and that $M$ generates properly disipative solutions of (1.16). Moreover, the domain of $M$ is determined by the $Q$-orthogonal complement of $N$ in $H$.

Boundary conditions of the elastic type are not included in the above development. In order to treat such boundary conditions we have coupled the system (1.2) at the boundary to a relatively simple "dissipative" system. As we shall see, the general theory developed in Chapter I applies as well to the coupled system.

The present work is an outgrowth of an earlier paper by the author [12] which treated the Cauchy problem (1.2) for the case of one spatial variable, in essence, by a green's function method. Much of the motivation for the ideas and concepts introduced here is to be found in [12]. However, the operator-theoretic approach is new and we feel that it has enabled us to treat (1.2) in the general case $(m \geqq 1)$ with much less work than was required by the previous method for the case $m=1$. On the other hand, we have not been able to obtain the wealth of detail which is to be found in [12]. K. Yosida [14] has also written a paper treating the wave equation from the semi-group point of view in which he establishes the existence of a single solution, assuming $\Delta$ to be the entire euclidean space; moreover Yosida has imposed rather severe regularity conditions on the coefficients of the wave equation. 


\section{Chapter I. Theory of Dissipative operators}

1.1. Basic properties of dissipative operators. The central problem treated in this chapter is the extension of dissipative operators to maximal dissipative operators. The existence of such a maximal dissipative extension is a simple consequence of the maximal principle; however, mere existence is by no means sufficient for our purposes. The present section treats of certain basic properties of dissipative operators obtained, for the most part, by means of a variant of the von Neumann-Cayley transform theory (see, for instance, $[11 ; \S \mathrm{VI} .3])$. This transform theory furnishes some insight into the extension problem by providing a construction for all possible maximal dissipative extensions of a given dissipative operator with dense domain in terms of corresponding extensions of an associated contraction operator.

Definition 1.1.1. If $L$ is a transformation on $H_{0}$ to itself, we denote the range of $L$ by $\Re(L)$ and the graph by $\$(L)$;

$$
\text { (S) }(L)=\left[\left[y^{0}, L y^{0}\right] ; y^{0} \in \mathfrak{D}(L)\right] \subset H_{0} \times H_{0} .
$$

Lemma 1.1.1. Let $L$ be a dissipative operator and suppose $\lambda>0$. Then for $f^{0}=\lambda y^{0}-L y^{0}, y^{0} \in \mathfrak{D}(L)$, we have

$$
\lambda\left\|y^{0}\right\| \leqq\left\|f^{0}\right\| .
$$

Further, the map $\left[y^{0}, L y^{0}\right] \rightarrow f^{0}$ of $\mathfrak{S}(L)$ onto $\Re(\lambda I-L)$ is one-to-one and bicontinuous.

Proof. It is clear that

$$
\begin{aligned}
2 \lambda\left\langle y^{0}, y^{0}\right\rangle & \leqq 2 \lambda\left\langle y^{0}, y^{0}\right\rangle-\left[\left\langle L y^{0}, y^{0}\right\rangle+\left\langle y^{0}, L y^{0}\right\rangle\right] \\
& =\left\langle f^{0}, y^{0}\right\rangle+\left\langle y^{0}, f^{0}\right\rangle \leqq 2\left\|y^{0}\right\|\left\|f^{0}\right\|
\end{aligned}
$$

from which (1.1.1) follows directly. Further, setting $c^{\prime}(\lambda)=\max (1, \lambda)$, we have

$$
\begin{aligned}
\left\|f^{0}\right\| \leqq c^{\prime}(\lambda)\left[\left\|y^{0}\right\|+\left\|L y^{0}\right\|\right] & \leqq c^{\prime}(\lambda)\left[\left\|y^{0}\right\|+\left(\lambda\left\|y^{0}\right\|+\left\|f^{0}\right\|\right)\right] \\
& \leqq c^{\prime}(\lambda)\left[2+\lambda^{-1}\right]\left\|f^{0}\right\|,
\end{aligned}
$$

and this proves the last assertion of the lemma.

We note that (1.1.1) implies that $\lambda I-L$ is one-to-one when $\lambda>0$ so that if $\Re(\lambda I-L)=H_{0}$, then $L$ is necessarily maximal dissipative. It also follows from the lemma that $L$ is closed if and only if $\Re(\lambda I-L)$ is closed.

We now define the operator

$$
J=(I+L)(I-L)^{-1} \text { with } \mathfrak{D}(J)=\Re(I-L) .
$$

It is easy to see that $J$ is a contraction operator. For, setting

$$
u^{0}=y^{0}-L y^{0} \text { and } J u^{0}=y^{0}+L y^{0},
$$$$
y^{0} \in \mathfrak{D}(L),
$$

we obtain 


$$
\begin{aligned}
\left\|J u^{0}\right\|^{2} & =\left\|y^{0}\right\|^{2}+\left\|L y^{0}\right\|^{2}+\left[\left\langle L y^{0}, y^{0}\right\rangle+\left\langle y^{0}, L y^{0}\right\rangle\right] \\
& \leqq\left\|y^{0}\right\|^{2}+\left\|L y^{0}\right\|^{2}-\left[\left\langle L y^{0}, y^{0}\right\rangle+\left\langle y^{0}, L y^{0}\right\rangle\right]=\left\|u^{0}\right\|^{2}
\end{aligned}
$$

here we have made use of the fact that $L$ is dissipative. Further solving the Equations (1.1.3) for $y^{0}$ and $L y^{0}$ we get

$$
y^{0}=\frac{1}{2}\left[J u^{0}+u^{0}\right] \quad \text { and } \quad L y^{0}=\frac{1}{2}\left[J u^{0}-u^{0}\right], \quad u^{0} \in \mathcal{D}(J),
$$

from which it follows that $I+J$ is one-to-one and that $\Re(I+J)=\mathfrak{D}(L)$. Since $J$ is bounded, it will be closed if and only if $\mathfrak{D}(J)=\Re(I-L)$ is closed and hence if and only if $L$ is closed.

Conversely, suppose $J$ is a given contraction operator such that $I+J$ is one-to-one. Then the operator $L$ defined by means of (1.1.4) is linear (and hence single-valued) with $\mathfrak{D}(L)=\mathfrak{R}(I+J)$. Moreover, for $y^{0} \in \mathfrak{D}(L)$ we have

$$
\begin{aligned}
\left\langle L y^{0}, y^{0}\right\rangle+\left\langle y^{0}, L y^{0}\right\rangle & =\frac{1}{4}\left[\left\langle\left(J u^{0}-u^{0}\right),\left(J u^{0}+u^{0}\right)\right\rangle+\left\langle\left(J u^{0}+u^{0}\right),\left(J u^{0}-u^{0}\right)\right\rangle\right] \\
& =\frac{1}{2}\left[\left\|J u^{0}\right\|^{2}-\left\|u^{0}\right\|^{2}\right] \leqq 0,
\end{aligned}
$$

so that $L$ is a dissipative operator.

Finally we show that $I+J$ will be one-to-one if $\Re(I+J)$ is dense in $H_{0}$. To this end, suppose that $J u^{0}+u^{0}=\theta$, let $v^{0}$ be any element of $\mathfrak{D}(J)$, and set $g^{0}=v^{0}+J v^{0}$. Then

which reduces to

$$
\left\|J\left(v^{0}-\alpha u^{0}\right)\right\|^{2} \leqq\left\|v^{0}-\alpha u^{0}\right\|^{2}
$$

$$
\alpha\left\langle u^{0}, g^{0}\right\rangle+\bar{\alpha}\left\langle g^{0}, u^{0}\right\rangle \leqq\left\|v^{0}\right\|^{2}-\left\|J v^{0}\right\|^{2} .
$$

Since $\alpha$ is arbitrary, it follows that $\left\langle u^{0}, g^{0}\right\rangle=0$ and by assumption this must hold for a dense set of $g^{0}$. Consequently $u^{0}=\theta$ and this proves that $I+J$ is one-to-one.

We note that the Banach-Steinhaus theorem provides any contraction operator $J$ with a unique extension of the same kind having the closure of $\mathfrak{D}(J)$ as its domain. On the other hand, a contraction operator $J$ with a closed domain can always be extended in a trivial fashion to a contraction operator $J_{1}$ on $H_{0}$ by setting $J_{1} y^{0}=\theta$ for all $y^{1} \in \mathfrak{D}(J) \perp$. We summarize these results in

THeOREm 1.1.1. If $L_{0}$ is a dissipative operator with dense domain, then the operator $J_{0}$ defined as in (1.1.3) is a contraction operator; $L_{0}$ and $J_{0}$ are closed together. Conversely, if $J_{0}$ is a contraction operator with $\Re\left(I+J_{0}\right)$ dense in $H_{0}$, then $L_{0}$ defined as in (1.1.4) is a dissipative operator with dense domain and $J_{0}$ is again given by (1.1.3). The relations (1.1.3) and (1.1.4) establish a one-toone inclusion preserving correspondence between all dissipative extensions $L$ of 
$L_{0}$ and all contraction extensions $J$ of $J_{0}$. In particular, the maximal dissipative extensions of $L_{0}$ correspond to the (closed) contraction extensions of $J_{0}$ with domains equal to $H_{0}$.

COROLlARy. Let $\lambda>0$ and suppose that $L$ is a dissipative operator with dense domain. Then $L$ is maximal dissipative if and only if $\Re(\lambda I-L)=H_{0}$.

Proof. It is clear that $L$ and $\lambda^{-1} L$ are dissipative and maximal dissipative together. It follows from the previous theorem that $\lambda^{-1} L$ is maximal dissipative if and only if $\Re\left(I-\lambda^{-1} L\right)=\Re(\lambda I-L)$ fills out $H_{0}$.

The nontrivial contraction extensions of $J_{0}$ are governed by the following.

Lemma 1.1.2. Let $J$ be a linear operator defined on the two-dimensional subspace generated by $y^{0}$ and $z^{0}$ where $\left\langle y^{0}, z^{0}\right\rangle=0$. A necessary and sufficient condition that $J$ be a contraction operator is that

$$
\left|\left\langle J y^{0}, J z^{0}\right\rangle\right|^{2} \leqq \delta y^{0} \delta z^{0}
$$

where $\delta u^{0}=\left\|u^{0}\right\|^{2}-\left\|J u^{0}\right\|^{2}$.

Proof. The operator $J$ will be a contraction operator if and only if

$$
\left\|J\left(\alpha y^{0}+\gamma z^{0}\right)\right\|^{2} \leqq\left\|\alpha y^{0}+\gamma z^{0}\right\|^{2}=|\alpha|^{2}\left\|y^{0}\right\|^{2}+|\gamma|^{2}\left\|z^{0}\right\|^{2}
$$

for all $\alpha, \gamma$. This is equivalent with

$$
|\alpha|^{2} \delta y^{0}+|\gamma|^{2} \delta z^{0}-\alpha \bar{\gamma}\left\langle J y^{0}, J z^{0}\right\rangle-\bar{\alpha} \gamma\left\langle J z^{0}, J y^{0}\right\rangle \geqq 0 .
$$

For fixed $|\alpha|$ and $|\gamma|$ the minimum of the left member is attained when $\arg (\alpha \bar{\gamma})=\arg \left(\left\langle J z^{0}, J y^{0}\right\rangle\right)$ in which case

$$
|\alpha|^{2} \delta y^{0}+|\gamma|^{2} \delta z^{0}-2|\alpha||\gamma|\left|\left\langle J y^{0}, J z^{0}\right\rangle\right| \geqq 0
$$

and this is easily seen to be true for all $|\alpha|,|\gamma|$ if and only if (1.1.5) is satisfied.

It follows from Theorem 1.1.1 that a maximal dissipative operator with dense domain is necessarily closed $\left({ }^{6}\right)$. We next prove a converse to this statement.

Lemma 1.1.3. If $L$ is maximal dissipative and closed, then it has a dense domain.

(6) The following is an example of a maximal dissipative operator $L$ which is not closed. In view of the above correspondence theory, it is clear that for the construction of such an $L$ it suffices to define a contraction operator $J$ with $I+J$ one-to-one which has no proper extension with these same properties, and yet such that $\mathfrak{D}(J)$ is not closed. To this end suppose that $\left\{\phi_{n}^{0}\right\}$ is a complete orthonormal system for $H_{0}$ and define $J_{1}$ with domain $H_{0}$ so that $J_{1} \phi_{1}^{0}=-\phi_{1}^{0}$ and $J_{1} \phi_{n}^{0}=\phi_{n}^{0}$ for all $n>1$. Then $\left(I+J_{1}\right) y^{0}=\theta$ if and only if $y^{0}=\alpha \phi_{1}^{0}$. Thus the above requirements for $J$ will be satisfied by any restriction of $J_{1}$ for which $\mathfrak{D}(J)$ is dense in $H_{0}$ and for which $\mathfrak{D}(J)$ is maximal with respect to the property of not including $\phi_{1}^{0}$. Now $\mathfrak{D}_{0} \equiv$ linear extension of $\left[\sum_{n-1}^{\infty} 2^{-n} \phi_{n}^{0}, \phi_{2}^{0}, \phi_{3}^{0}, \cdots\right]$ is dense in $H_{0}$ and does not include $\phi_{1}^{0}$. Applying the maximal principle to the subspaces of $H_{0}$ containing $\mathfrak{D}_{0}$ yet not containing $\phi_{1}^{0}$, yields a domain with the desired properties. 
Proof. We first show that $\Re(I-L)=H_{0}$. To this end we define $J$ as in (1.1.3). Then $J$ is a contraction operator with $\mathscr{D}(J)=\Re(I-L)$. Since $L$ is a closed operator, Lemma 1.1.1 implies that $\Re(I-L)$ is a closed subspace. Consequently if $\Re(I-L)$ is a proper subspace of $H_{0}$ there exists a nonzero vector $u^{0} \in \mathfrak{D}(J) \perp$. We can therefore extend $J$ to a contraction operator $J_{1}$ by setting $J_{1}\left(y^{0}+\alpha u^{0}\right)=J y^{0}$ for all $y^{0} \in \mathfrak{D}(J)$. Now $I+J_{1}$ is one-to-one. For suppose $J_{1}\left(y^{0}+\alpha u^{0}\right)=-\left(y^{0}+\alpha u^{0}\right)$. Since $I+J$ is one-to-one we see that $\alpha \neq 0$ and hence that

$$
\left\|y^{0}+\alpha u^{0}\right\|=\left\|J_{1}\left(y^{0}+\alpha u^{0}\right)\right\|=\left\|J y^{0}\right\| \leqq\left\|y^{0}\right\|<\left\|y^{0}+\alpha u^{0}\right\|,
$$

which is impossible. It follows that $I+J_{1}$ is one-to-one and hence that $J_{1}$ defines a proper dissipative extension of $L$ as in (1.1.4). Since $L$ was assumed to be maximal dissipative, we conclude that $\Re(I-L)=\mathfrak{I}(J)=H_{0}$. Next, suppose that $\mathfrak{I}(L)=\Re(I+J)$ is not dense in $H_{0}$. Then the adjoint of $I+J$ takes a nonzero vector, say $v^{0}$, into zero; that is, $J^{*} v^{0}=-v^{0}$. Now $J^{*}$ is obviously a contraction operator along with $J$. Applying Lemma 1.1.2 to $J^{*}$ (with $\left.\delta v^{0}=0\right)$, we see that $\left\langle J^{*} y^{0}, v^{0}\right\rangle=-\left\langle J^{*} y^{0}, J^{*} v^{0}\right\rangle=0$ whenever $y^{0}$ is orthogonal to $v^{0}$. It follows from this that $J v^{0}=-v^{0}$, contrary to $I+J$ being one-to-one. We therefore conclude that $\mathfrak{D}(L)=\Re(I+J)$ is dense in $H_{0}$.

THEOREM 1.1.2. If $L$ is a maximal dissipative operator with dense domain, then so is $L^{*}$, and if $L$ corresponds as in Theorem 1.1.1 to the contraction operator $J$, then $L^{*}$ corresponds to the contraction operator $J^{*}$.

Proof. Since $L$ is maximal dissipative with dense domain, the corresponding contraction operator $J$ will have $H_{0}$ for its domain and $\Re(I+J)$ will be dense. Hence $J^{*}$ is well-defined and it is obviously a contraction operator. Moreover, $\Re\left(I+J^{*}\right)$ is also dense in $H_{0}$. If this were not so, then its adjoint, $I+J$, would take a nonzero vector into $\theta$. However, as we have seen in the proof of Theorem 1.1.1, this is contrary to $\Re(I+J)$ being dense. Consequently $\Re\left(I+J^{*}\right)$ is dense and $J^{*}$ corresponds as in Theorem 1.1.1 to a maximal dissipative operator, say $M$, with dense domain. Further $\left\langle J u^{0}, v^{0}\right\rangle=\left\langle u^{0}, J^{*} v^{0}\right\rangle$ implies

$$
\left\langle J u^{0}-u^{0}, J^{*} v^{0}+v^{0}\right\rangle=\left\langle J u^{0}+u^{0}, J^{*} v^{0}-v^{0}\right\rangle
$$

and hence $\left\langle L y^{0}, z^{0}\right\rangle=\left\langle y^{0}, M z^{0}\right\rangle$ for all $y^{0} \in \mathfrak{D}(L), z^{0} \in \mathfrak{D}(M)$. As a consequence $M \subset L^{*}$. We further note that $\Re(I-M)=\mathfrak{D}\left(J^{*}\right)=H_{0}$. Thus if $L^{*}$ were a proper extension of $M$, then $I-L^{*}$ would take a nonzero vector into $\theta$ and this is impossible when the range of its adjoint, $I-L$, equals $H_{0}$.

Corollary. Let $L_{0}$ be a dissipative operator with dense domain and set $M_{1}=L_{0}^{*}$. Then there exist maximal dissipative operators $L$ and $M$, adjoints of each other and both having dense domains, such that

$$
L \supset L_{0} \text { and } M \subset M_{1} \text {. }
$$


Proof. Let $L$ be any maximal dissipative operator extending $L_{0}$ and set $M=L^{*}$. According to the above theorem $M$ is again maximal dissipative with dense domain. Finally, since $L_{0} \subset L$, it follows that $M=L^{*} \subset L_{0}^{*}=M_{1}$.

The next theorem exhibits the importance of maximal dissipative operators in the theory of semi-groups.

THEOREM 1.1.3. A necessary and sufficient condition for an operator $L$ to generate a strongly continuous semi-group of contraction operators on $H_{0}$ to itself is that $L$ be a maximal dissipative operator with dense domain.

Proof. If $L$ is the infinitesimal generator of a strongly continuous semigroup of contraction operators, say $[S(t) ; t \geqq 0]$, then $\left\|S(t) y^{0}\right\| \leqq\left\|y^{0}\right\|$ and for $y^{0} \in \mathfrak{D}(L)$

$$
\left\langle y^{0}, L y^{0}\right\rangle+\left\langle L y^{0}, y^{0}\right\rangle=\left.\frac{d}{d t}\left\langle S(t) y^{0}, S(t) y^{0}\right\rangle\right|_{t=0} \leqq 0 .
$$

It follows that $L$ is dissipative. According to the general theory of semigroups (see, for instance, Hille and Phillips [7]), $\mathfrak{D}(L)$ is dense in $H_{0}$ and the resolvent set of $L$ contains all real $\lambda>0$. This implies, in particular, that $\Re(\lambda I-L)=H_{0}$ for $\lambda>0$ so that $L$ is maximal dissipative by the corollary to Theorem 1.1.1. Conversely, if $L$ is maximal dissipative with dense domain and $\lambda>0$, then the same corollary implies that $\Re(\lambda I-L)=H_{0}$. This together with the inequality (1.1.1) shows that the resolvent of $L$, namely $\Re(\lambda ; L)$ $=(\lambda I-L)^{-1}$, exists and satisfies the inequality $\|\lambda \Re(\lambda ; L)\|_{0} \leqq 1, \lambda>0$. Thus the hypothesis of the Hille-Yosida theorem (see [7]) is satisfied, and it follows that $L$ generates a strongly continuous semi-group of contraction operators.

Semi-groups of isometries form a particularly interesting subclass of the semi-groups of contraction operators since they correspond to energy conserving solutions of the Cauchy problem. They were first studied by J. L. B. Cooper $[2 ; 3]$ who gave a penetrating characterization of them. As we shall see, the generators of such semi-groups satisfy the following property.

Definition 1.1.2. An operator $L$ is called conservative if

$$
\left\langle L y^{0}, y^{0}\right\rangle+\left\langle y^{0}, L y^{0}\right\rangle=0,
$$$$
y^{0} \in \mathfrak{D}(L) .
$$

LEMMA 1.1.4. The operator $L$ is conservative if and only if iL is symmetric. If $L$ is conservative and maximal dissipative with dense domain, then iL is maximal symmetric. Conversely, if $i L$ is maximal symmetric with dense domain, then either $L$ or $-L$ is conservative and maximal dissipative. Finally, $L$ and $L^{*}$ are conservative with dense domains if and only if iL is self-adjoint.

Proof. If $L$ is conservative, then replacing $y^{0}$ in (1.1.6) by $u^{0}+\epsilon v^{0}, \epsilon= \pm 1$, $\pm i$, in turn and combining the resulting relations in the usual way, one obtains

$$
\left\langle L u^{0}, v^{0}\right\rangle+\left\langle u^{0}, L v^{0}\right\rangle=0
$$$$
u^{0}, v^{0} \in \mathfrak{D}(L) .
$$ 
It follows that $i L$ is symmetric whenever $L$ is conservative; the converse assertion is obvious. On the other hand if $L$ is conservative and maximal dissipative with dense domain, then $\Re(i I-i L)=\Re(I-L)=H_{0}$ by the corollary to Theorem 1.1.1. This shows that one of the deficiency indices of $i L$ is zero and hence that $i L$ is maximal symmetric. Conversely if $i L$ is maximal symmetric with dense domain then $\Re(I \pm L)=\Re(i I \pm i L)=H_{0}$ for one of the indicated signs and since $L$ and $-L$ are conservative together it follows from the above cited corollary that one of these operators is maximal dissipative. Finally if $L$ and $L^{*}$ are conservative with dense domains, then $i L$ and $(i L)^{*}$ $=-i L^{*}$ are symmetric and hence $i L$ is self-adjoint; again the converse is obvious.

LEMMA 1.1.5. Suppose $L_{0}$ is a closed conservative operator with dense domain and set $L_{1}=-L_{0}^{*}$. Then each maximal dissipative extension of $L_{0}$ is a restriction of $L_{1}$.

Proof. Since $i L_{0}$ is symmetric, it is clear that $L_{0} \subset L_{1}$. Suppose $L$ is a maximal dissipative extension of $L_{0}$ and let $J_{0}$ and $J$ be the contraction operators corresponding to $L_{0}$ and $L$, respectively, as in Theorem 1.1.1. Then $J$ is an extension of $J_{0}$ with $\mathfrak{D}(J)=H_{0}$, and it is readily seen that $J_{0}$ is an isometry with closed domain. Hence by Lemma 1.1.2, $v^{0}=J u^{0} \in \Re\left(J_{0}\right)^{\perp}$ whenever $u^{0} \in \mathfrak{D}\left(J_{0}\right)^{\perp}$. In other words, if

$$
\left\langle y^{0}-L_{0} y^{0}, u^{0}\right\rangle=0, \quad y^{0} \in \mathfrak{D}\left(L_{0}\right),
$$

then

$$
\left\langle y^{0}+L_{0} y^{0}, v^{0}\right\rangle=0,
$$$$
y^{0} \in \mathfrak{D}\left(L_{0}\right) .
$$

In this case $L_{1} u^{0}=-L_{0}^{*} u^{0}=-u^{0}$ and $L_{1} v^{0}=-L_{0}^{*} v^{0}=v^{0}$ so that by (1.1.4)

$$
L\left(v^{0}+u^{0}\right)=v^{0}-u^{0}=L_{1}\left(v^{0}+u^{0}\right) .
$$

Since $\mathfrak{D}(L)$ is the linear extension of $\mathfrak{D}\left(L_{0}\right)$ and vectors of the kind $\left(v^{0}+u^{0}\right)$, it follows that $L \subset L_{1}$.

The next theorem is due to Cooper [3], however the argument is new.

TheOREM 1.1.4. A necessary and sufficient condition for an operator $L$ to generate a strongly continuous semi-group of isometries is that $L$ be conservative and maximal dissipative with dense domain.

Proof. If $[S(t)]$ is a semi-group of isometries, then $\left\|S(t) y^{0}\right\|=\left\|y^{0}\right\|$ for all $t \geqq 0$. Thus for $y^{0} \in \mathfrak{D}(L)$

$$
\left\langle L y^{0}, y^{0}\right\rangle+\left\langle y^{0}, L y^{0}\right\rangle=\left.\frac{d}{d t}\left\langle S(t) y^{0}, S(t) y^{0}\right\rangle\right|_{t=0}=0
$$

and we see that $L$ is conservative. In addition, it follows from Theorem 1.1.3 that $L$ is also maximal dissipative with dense domain. Conversely, suppose 
$L$ is known to be conservative and maximal dissipative with dense domain. Then Theorem 1.1.3 also asserts that $L$ generates a strongly continuous semigroup of contraction operators, say $[S(t)]$. For $y^{0} \in \mathfrak{D}(L), S(t) y^{0}$ lies in $\mathfrak{D}(L)$ for all $t>0$ and, making use of the fact that $L$ is conservative, we get

$$
\frac{d}{d t}\left\langle S(t) y^{0}, S(t) y^{0}\right\rangle=\left\langle L S(t) y^{0}, S(t) y^{0}\right\rangle+\left\langle S(t) y^{0}, L S(t) y^{0}\right\rangle=0 .
$$

Consequently $\left\|S(t) y^{0}\right\|=\left\|y^{0}\right\|, t>0$, for each $y^{0} \in \mathfrak{D}(L)$ and since $\mathfrak{D}(L)$ is dense in $H_{0}$, this relation holds for all $y^{0} \in H_{0}$; thus $S(t)$ is an isometry. This completes the proof.

THEOREM 1.1.5. A necessary and sufficient condition for a conservative maximal dissipative operator with dense domain to generate a strongly continuous group is that iL be self-adjoint. In this case the group consists of unitary operators.

The argument can be taken verbatim from [12, Theorem 8.2].

1.2. Dual hilbert spaces. The theory which we are about to present requires two auxiliary dual hilbert spaces in addition to the basic hilbert space $H_{0}$. These are defined by means of a positive definite self-adjoint operator $F$ with (dense) domain $\mathfrak{D}(F)$ satisfying the condition

$$
\left\langle F y^{0}, y^{0}\right\rangle \geqq\left\langle y^{0}, y^{0}\right\rangle,
$$$$
y^{0} \in \mathfrak{D}(F) .
$$

It is clear that $F^{-1}$ exists and is a positive definite operator with domain $H_{0}$ and norm $\leqq 1$.

We now define two new inner products

$$
\left\langle y^{0}, z^{0}\right\rangle_{1} \equiv\left\langle F y^{0}, z^{0}\right\rangle,
$$$$
y^{0}, z^{0} \in \mathfrak{D}(F),
$$

and

$$
\left\langle y^{0}, z^{0}\right\rangle_{2} \equiv\left\langle F^{-1} y^{0}, z^{0}\right\rangle,
$$$$
y^{0}, z^{0} \in H_{0} .
$$

Finally $H_{1}$ and $H_{2}$ are the respective completions of the two pre-hilbert spaces defined by these inner products. A Cauchy sequence consisting of repetitions of a fixed element $y^{0}$ of $\mathfrak{D}(F)$ will, of course, define an element, say $y^{1}$, of $H_{1}$ and for the sake of clarity we will write $y^{0}=I_{01} y^{1}$. Likewise a Cauchy sequence consisting of a single element $y^{0}$ of $H_{0}$ will define an element $y^{2}$ in $H_{2}$ and we shall write $y^{2}=I_{20} y^{0}$. Both of these maps are one-to-one.

LEMMA 1.2.1. The map $I_{01}$ can be uniquely extended to be one-to-one on $H_{1}$ into $H_{0}$ and of norm $\leqq 1$. Similarly the map $I_{20}$ is one-to-one and of norm $\leqq 1$.

Proof. The elements of $H_{1}$ are defined in the usual manner as classes of Cauchy sequences in $\mathfrak{D}(F)$. Suppose that $\left\{y_{n}^{0}\right\}$ is such a sequence defining an element $y^{1}$ of $H_{1}$. Then 


$$
\left\|y_{n}^{0}-y_{m}^{0}\right\| \leqq\left\|y_{n}^{0}-y_{m}^{0}\right\|_{1} \rightarrow 0
$$

as $n, m \rightarrow \infty$. Thus $\left\{y_{n}^{0}\right\}$ is also a Cauchy sequence in $H_{0}$ and converges to some element, say $y^{0} \in H_{0}$. We define $I_{01} y^{1}=y^{0}$. It is clear that this extends our previously defined $I_{01}$ and that the so-defined $I_{01}$ is linear and of norm $\leqq 1$. To show that the extended $I_{01}$ is one-to-one, we suppose that the above $y^{0}=\theta$. Then for arbitrary $z^{0} \in \mathcal{D}(F)$ with $z^{1}=I_{01}^{-1} z^{0}$, we have

$$
\left\langle y^{1}, z^{1}\right\rangle_{1}=\lim _{n \rightarrow \infty}\left\langle F y_{n}^{0}, z^{0}\right\rangle=\lim _{n \rightarrow \infty}\left\langle y_{n}^{0}, F z^{0}\right\rangle=\left\langle y^{0}, F z^{0}\right\rangle=0 .
$$

Thus $\left\langle y^{1}, z^{1}\right\rangle_{1}=0$ for a set of $z^{1}$ dense in $H_{1}$ and it follows that $y^{1}=\theta$. The uniqueness of this extension is a consequence of the fact that $I_{01}^{-1}[\mathfrak{D}(F)]$ is dense in $H_{1}$. The assertions about $I_{20}$ follow directly from the properties of $F^{-1}$.

Lemma 1.2.2. There is a unitary map of $\mathrm{H}_{1}$ onto $\mathrm{H}_{2}$ which takes the Cauchy sequence $\left\{y_{n}^{0}\right\} \subset \mathfrak{D}(F)$, defining an element of $H_{1}$, into the Cauchy sequence $\left\{F y_{n}^{0}\right\}$, defining an element of $H_{2}$. This map can be thought of as an extension of $F$ and we shall denote it by $F_{21}$, with $F_{12}=F_{21}^{-1}$. For $y^{1} \in H_{1}$ and $z^{2} \in H_{2}$ we define

$$
\left\langle y^{1}, z^{2}\right\rangle \equiv\left\langle y^{1}, F_{12} z^{2}\right\rangle_{1}=\left\langle F_{21} y^{1}, z^{2}\right\rangle_{2} .
$$

For Cauchy sequences $\left\{y_{n}^{0}\right\} \subset \mathcal{D}(F)$ defining $y^{1} \in H_{1}$ and $\left\{z_{\boldsymbol{k}}^{0}\right\} \subset H_{0}$ defining $z^{2} \in H_{2}$ we have

$$
\left\langle y^{1}, z^{2}\right\rangle=\lim _{n, k \rightarrow \infty}\left\langle y_{n}^{0}, z_{k}^{0}\right\rangle
$$

In particular if $y^{1}=I_{01}^{-1} y^{0}$ and $z^{2}=I_{20} z^{0}$, then $\left\langle y^{1}, z^{2}\right\rangle=\left\langle y^{0}, z^{0}\right\rangle$.

Proof. The unitary character of $F_{12}$ follows immediately from two relations

$$
\left\langle F y_{n}^{0}, F y_{n}^{0}\right\rangle_{2}=\left\langle F^{-1}\left(F y_{n}^{0}\right), F y_{n}^{0}\right\rangle=\left\langle F y_{n}^{0}, y_{n}^{0}\right\rangle=\left\langle y_{n}^{0}, y_{n}^{0}\right\rangle_{1}
$$

and

$$
\left\langle z_{n}^{0}, z_{n}^{0}\right\rangle_{2}=\left\langle F^{-1} z_{n}^{0}, z_{n}^{0}\right\rangle=\left\langle F\left(F^{-1} z_{n}^{0}\right), F^{-1} z_{n}^{0} e\right\rangle=\left\langle F^{-1} z_{n}^{0}, F_{1}^{-1} z_{n}^{0}\right\rangle_{1} .
$$

Further, since $\left\{F^{-1} z_{k}^{0}\right\}$ defines the element $F_{12} z^{2}$ in $H_{1}$, we have

$$
\left\langle y^{1}, z^{2}\right\rangle \equiv\left\langle y^{1}, F_{12} z^{2}\right\rangle_{1}=\lim _{n, k \rightarrow \infty}\left\langle F y_{n}^{0}, F^{-1} z_{k}^{0}\right\rangle=\lim _{n, k \rightarrow \infty}\left\langle\stackrel{0}{y_{n}}, z_{k}^{0}\right\rangle .
$$

By choosing $z_{k}^{0}=z^{0}$ for all $k$, we see that $z^{2}=I_{20} z^{0}$ and $y^{0} \equiv \lim _{n} y_{n}^{0}=I_{01} y^{1}$, which proves the last assertion of the lemma.

One sees from the unitary nature of $F_{12}$ that

$$
\left|\left\langle y^{1}, z^{2}\right\rangle\right|=\left|\left\langle y^{1}, F_{12} z^{2}\right\rangle_{1}\right| \leqq\left\|y^{1}\right\|_{1}\left\|F_{12} z^{2}\right\|_{1}=\left\|y^{1}\right\|{ }_{1}\left\|z^{2}\right\|_{2} .
$$


Definition 1.2.1. Let $C_{21}$ be a bounded linear transformation on $H_{1}$ to $H_{2}$. Then $C_{21}$ will be called symmetric if $\left\langle C_{21} y^{1}, z^{1}\right\rangle=\left\langle y^{1}, C_{21} z^{1}\right\rangle$ for all $y^{1}, z^{1} \in H_{1}$, and it will be called positive if $\left\langle C_{21} y^{1}, y^{1}\right\rangle \geqq 0$ for all $y^{1} \in H_{1}$, in symbols, $C_{21} \geqq \Theta$.

Lemma 1.2.3. Let $C$ be a linear operator on $H_{0}$ with $\mathfrak{D}(C)=\mathfrak{D}(F)$ and such that

$$
\left\langle F^{-1} C y^{0}, C y^{0}\right\rangle \leqq k\left\langle F y^{0}, y^{0}\right\rangle, \quad \quad y^{0} \in \mathfrak{D}(F) .
$$

Then $C$ takes each Cauchy sequence $\left\{y_{n}^{0}\right\} \subset \mathfrak{D}(F)$, defining an element of $H_{1}$, into a Cauchy sequence $\left\{C y_{n}^{0}\right\}$, defining an element of $H_{2}$, and in this way defines a bounded linear transformation $C_{21}$ on $H_{1}$ to $H_{2}$ of norm $\leqq k^{1 / 2}$. If $C$ is symmetric (or positive), then $C_{21}$ will likewise be symmetric (or positive).

Proof. The condition (1.2.6) implies that $\left\{C y_{n}^{0}\right\}$ is a Cauchy sequence defining an element of $\mathrm{H}_{2}$ and hence that

$$
\left\langle C_{21} y^{1}, C_{21} y^{1}\right\rangle_{2}=\lim _{n \rightarrow \infty}\left\langle F^{-1} C y_{n}^{0}, C y_{n}^{0}\right\rangle \leqq k \lim _{n \rightarrow \infty}\left\langle F y_{n}^{0}, y_{n}^{0}\right\rangle \leqq k\left\langle y^{1}, y^{1}\right\rangle_{1} .
$$

It follows from this that $C_{21}$ is a linear transformation on $H_{1}$ to $H_{2}$ of bound $\leqq k^{1 / 2}$. Let $y^{1}$ and $z^{1}$ be defined by the Cauchy sequences $\left\{y_{n}^{0}\right\}$ and $\left\{z_{n}^{0}\right\}$ $C \mathfrak{D}(F)$, respectively. Then if $C$ is symmetric

$$
\left\langle C_{21} y^{1}, z^{1}\right\rangle=\lim _{n \rightarrow \infty}\left\langle C y_{n}^{0}, z_{n}^{0}\right\rangle=\lim _{n \rightarrow \infty}\left\langle y_{n}^{0}, C z_{n}^{0}\right\rangle=\left\langle y^{1}, C_{21} z^{1}\right\rangle
$$

whereas if $C$ is positive

$$
\left\langle C_{21} y^{1}, y^{1}\right\rangle=\lim _{n \rightarrow \infty}\left\langle C y_{n}^{0}, y_{n}^{0}\right\rangle \geqq 0 .
$$

Corollary. The operators $I, D \equiv I-F$, and $F(\lambda) \equiv \lambda I-D, \lambda>0$, each with domain $\mathfrak{D}(F)$, have bounded linear extensions on $H_{1}$ to $H_{2}$. If we denote these extensions by $I_{21}, D_{21}$ and $F(\lambda)_{21}$, respectively, then $I_{21}=I_{20} I_{01}, D_{21}=I_{21}$ $-F_{21}$, and $F(\lambda)_{21}=\lambda I_{21}-D_{21}$. Further $I_{21},-D_{21}$ and $F(\lambda)_{21}$ are positive symmetric.

Proof. Let $C$ denote $I,-D$, and $F(\lambda)$ in turn. In each case $C$ is positive symmetric and there exists a $k$ such that

$$
0 \leqq\left\langle C y^{0}, y^{0}\right\rangle \leqq k^{1 / 2}\left\langle F y^{0}, y^{0}\right\rangle, \quad y^{0} \in \mathfrak{D}(F) .
$$

Applying the Schwarz inequality with respect to the inner product $\left\langle C z^{0}, z^{0}\right\rangle$, we obtain

$$
\begin{aligned}
{\left[\left\langle F^{-1}\left(C y^{0}\right), C y^{0}\right\rangle\right]^{2} } & \leqq\left\langle C y^{0}, y^{0}\right\rangle\left\langle C F^{-1} C y^{0}, F^{-1} C y^{0}\right\rangle \\
& \leqq k\left\langle F y^{0}, y^{0}\right\rangle\left\langle F F^{-1} C y^{0}, F^{-1} C y^{0}\right\rangle
\end{aligned}
$$


and this implies (1.2.6). Thus the operators have bounded linear extensions on $H_{1}$ to $H_{2}$ and the asserted relations follow if we merely consider the Cauchy sequences used in defining these extensions. We note, incidently, for both $I_{21}$ and $-D_{21}$ that $k \leqq 1$.

1.3. Negative subspaces. Next let $H_{12}=H_{1} \times H_{2}$ denote the product space of elements $y=\left[y^{1}, y^{2}\right], y^{1} \in H_{1}$ and $y^{2} \in H_{2}$ with inner product

$$
\langle y, z\rangle=\left\langle y^{1}, z^{1}\right\rangle_{1}+\left\langle y^{2}, z^{2}\right\rangle_{2} .
$$

When $F=I$ we note that $H_{1}=H_{0}=H_{2}$ and $H_{12}$ becomes $H_{0} \times H_{0}$.

Definition 1.3.1. If $L_{21}$ is a transformation on $H_{1}$ to $H_{2}$ with domain $\mathfrak{I}\left(L_{21}\right)$, then its graph is a subspace of $H_{12}$ defined as

$$
\mathfrak{S}\left(L_{21}\right)=\left[\left[y^{1}, L_{21} y^{1}\right] ; y^{1} \in \mathfrak{D}\left(L_{21}\right)\right] .
$$

As we shall see, the graphs of the dissipative operators in which we are interested correspond to negative subspaces of $H_{12}$ relative to the hermitian symmetric bilinear form

$$
Q(y, z) \equiv\left\langle y^{1}, z^{2}\right\rangle+\left\langle y^{2}, z^{1}\right\rangle-\left\langle D_{21} y^{1}, z^{1}\right\rangle .
$$

It is at any rate clear in the case $F=I$ (and hence $D_{21}=\Theta$ ) that the graph of a dissipative operator is negative.

It is easy to see that $Q$ is a continuous form; in fact

$$
|Q(y, z)| \leqq\left\|y^{1}\right\|{ }_{1}\left\|z^{2}\right\|_{2}+\left\|y^{2}\right\|_{2}\left\|z^{1}\right\|_{1}+\left\|y^{1}\right\|{ }_{1}\left\|z^{1}\right\|_{1} \leqq 2\|y\|\|z\| .
$$

The continuity of $Q$ shows that the closure of a negative (or positive) subspace is again negative (or positive). It follows from the maximal principle that each negative (positive) subspace is contained in a maximal negative (maximal positive) subspace, which is necessarily closed since otherwise its closure would again be negative (positive). Moreover the continuity of $Q$ also requires that the $Q$-orthogonal complement of a set be a closed linear subspace.

It is convenient at this point to introduce the following operators on $H_{12}$ to itself:

$$
\begin{aligned}
U\left[y^{1}, y^{2}\right] & =\left[y^{1},-y^{2}+D_{21} y^{1}\right], \\
V\left[y^{1}, y^{2}\right] & =\left[F_{12} y^{2},-F_{21} y^{1}\right], \\
W\left[y^{1}, y^{2}\right] & \equiv U V\left[y^{1}, y^{2}\right]=\left[F_{12} y^{2}, F_{21} y^{1}+D_{21} F_{12} y^{2}\right] .
\end{aligned}
$$

The corollary to Lemma 1.2.3 shows that $U, V$, and $W$ are all linear bounded operators. Moreover

$$
U^{2}=I \quad \text { and } \quad V^{2}=-I
$$

so that the inverses of these operators are likewise bounded operators on $H_{12}$. A simple calculation gives 


$$
\begin{aligned}
Q(U y, U z) & =-Q(y, z) \\
Q(y, U z) & =-\left\langle y^{1}, z^{2}\right\rangle+\left\langle y^{2}, z^{1}\right\rangle \\
Q(W y, z) & =Q(y, W z)=\left\langle F_{21} y^{1}, z^{1}\right\rangle+\left\langle F_{12} y^{2}, z^{2}\right\rangle=\langle y, z\rangle .
\end{aligned}
$$

The first of the identities (1.3.6) shows that the mapping $y \rightarrow U y$ of $H_{12}$ on itself defines a one-to-one correspondence between the negative subspaces and the positive subspaces and it is clear that this correspondence preserves inclusion and $Q$-orthogonality. As a consequence, a statement about negative subspaces will, in general, imply a dual statement about positive subspaces.

DEFINITION 1.3.2. Given a subset $S \subset H_{12}$, we define its domain $\mathfrak{D}(S)$ and range $\Re(S)$ by

$$
\begin{aligned}
& \mathfrak{D}(S)=\left[y^{1} ;\left[y^{1}, y^{2}\right] \in S\right], \\
& \Re(S)=\left[y^{2} ;\left[y^{1}, y^{2}\right] \in S\right] .
\end{aligned}
$$

We further set

$$
\Re_{\lambda}(S)=\left[\lambda I_{21} y^{1}-y^{2} ;\left[y^{1}, y^{2}\right] \in S\right] .
$$

A linear subspace $S$ of $H_{12}$ is the graph of a linear transformation on $H_{1}$ to $H_{2}$ if and only if $S$ contains no element $y=\left[y^{1}, y^{2}\right]$ of the form $y^{1}=\theta$, $y^{2} \neq \theta$. It follows from this that $S$ is a graph if and only if $U[S]$ is a graph. We note that $\mathfrak{D}(S)=\mathfrak{D}(U[S])$. The graph of a closed linear transformation is by definition a closed subspace. It should be observed that the graph of a linear transformation on $H_{1}$ to $H_{2}$ may be negative and the transformation may even be maximal in this respect, yet the graph itself may be the proper subset of some other negative subspace. As the following lemma shows, this situation will not occur if the domain of the transformation is dense in $H_{1}$.

Lemma 1.3.1. A negative (or positive) subspace with dense domain is the graph of a linear transformation on $\mathrm{H}_{1}$ to $\mathrm{H}_{2}$.

Proof. From the above remarks, it is clear that it suffices to consider only the case of a negative subspace $N$ with dense domain. Suppose there were a $u=\left[\theta, u^{2}\right] \in N, u^{2} \neq \theta$; we note that $Q(u, u)=0$. Since $\mathfrak{D}(N)$ is dense in $H_{1}$, there is a $y=\left[y^{1}, y^{2}\right] \in N$ such that $\left\langle y^{1}, u^{2}\right\rangle=\left\langle y^{1}, F_{12} u^{2}\right\rangle_{1} \neq 0$. Now $y+\alpha u \in N$ and hence

$$
Q(y+\alpha u, y+\alpha u)=Q(y, y)+\alpha Q(u, y)+\bar{\alpha} Q(y, u) \leqq 0 .
$$

As this holds for all $\alpha$, we conclude that $Q(y, u)=\left\langle y^{1}, u^{2}\right\rangle$ must equal zero, contrary to our choice of $y$.

Corollary. If the negative (positive) subspace $N$ is the graph of a linear transformation on $H_{1}$ to $H_{2}$ with dense domain, then the same is true of each maximal negative (maximal positive) subspace containing $N$.

Next we obtain some properties of negative subspaces. 
Lemma 1.3.2. Let $N$ be a negative subspace and suppose $\lambda>0$. Then for $f^{2}=\lambda I_{21} y^{1}-y^{2},\left[y^{1}, y^{2}\right] \in N$, we have

$$
c(\lambda)\left\|y^{1}\right\|_{1} \leqq\left\|f^{2}\right\|_{2},
$$

where $c(\lambda)=\min (1 / 2, \lambda)$.

Proof. We obtain directly from the definition of $f^{2}$ that $\lambda\left\langle I_{21} y^{1}, y^{1}\right\rangle$ $-\left\langle y^{2}, y^{1}\right\rangle=\left\langle f^{2}, y^{1}\right\rangle$ and adding this to its complex conjugate we get

$$
\text { (1.3.8) } 2 \lambda\left\langle I_{21} y^{1}, y^{1}\right\rangle-\left\langle D_{21} y^{1}, y^{1}\right\rangle-Q(y, y)=\left\langle y^{1}, f^{2}\right\rangle+\left\langle f^{2}, y^{1}\right\rangle \leqq 2\left\|y^{1}\right\|_{1}\left\|f^{2}\right\|_{2} \text {. }
$$

According to the corollary to Lemma 1.2 .3 both $I_{21}$ and $-D_{21}$ are positive symmetric and $F_{21}=I_{21}-D_{21}$; this together with the fact that $Q(y, y) \leqq 0$ for $y \in N$ implies

$$
c(\lambda)\left\|y^{1}\right\|_{1}^{2}=c(\lambda)\left\langle F_{21} y^{1}, y^{1}\right\rangle \leqq\left\|y^{1}\right\|_{1}\left\|f^{2}\right\|_{2},
$$

so that (1.3.7) follows.

Lemma 1.3.3. Let $\lambda>0$ and suppose $N$ is a negative subspace. Then the mapping

$$
y=\left[y^{1}, y^{2}\right] \rightarrow f^{2} \equiv \lambda I_{21} y^{1}-y^{2}
$$

of $N$ onto $\Re_{\lambda}(N)$ is one-to-one and bicontinuous.

Proof. Making use of the inequality (1.3.7) we have

$$
\begin{aligned}
& \left\|y^{1}\right\|_{1} \leqq[c(\lambda)]^{-1}\left\|f^{2}\right\|_{2}, \\
& \left\|y^{2}\right\|_{2} \leqq\left\|f^{2}\right\|_{2}+\lambda\left\|I_{21} y^{1}\right\|_{2} \leqq\left\|f^{2}\right\|_{2}+\lambda\left\|y^{1}\right\|_{1} \leqq[1+\lambda / c(\lambda)]\left\|f^{2}\right\|_{2},
\end{aligned}
$$

and

$$
\left\|f^{2}\right\|_{2} \leqq \lambda\left\|I_{21} y^{1}\right\|_{2}+\left\|y^{2}\right\|_{2} \leqq \lambda\left\|y^{1}\right\|_{1}+\left\|y^{2}\right\|_{2} \leqq c^{\prime}(\lambda)\left[\left\|y^{1}\right\|_{1}+\left\|y^{2}\right\|_{2}\right],
$$

where $c^{\prime}(\lambda)=\max (1, \lambda)$. The result now follows directly.

Corollary. Let $\lambda>0$. Then a negative subspace $N$ is closed if and only if $\Re_{\lambda}(N)$ is a closed subspace of $H_{2}$.

Lemma 1.3.4. Let $\lambda>0$ and suppose $N$ is a negative subspace. Then $N$ is maximal negative if and only if $\mathfrak{R}_{\lambda}(N)=H_{2}$.

Proof. Suppose first that $N$ is maximal negative but that $\Re_{\lambda}(N)$ is a proper subspace of $H_{2}$. Since $N$ is closed, we see from the previous lemma that $\Re_{\lambda}(N)$ is also closed. Thus there is a $u^{2} \neq \theta$ such that $\left\langle F_{12} u^{2}, f^{2}\right\rangle=0$ for all $f^{2} \in \Re_{\lambda}(N)$. Now we set

$$
z^{1}=F_{12} u^{2}, \quad z^{2}=-F(\lambda)_{21} F_{12} u^{2}, \quad z=\left[z^{1}, z^{2}\right],
$$

where $F(\lambda)_{21}=\lambda I_{21}-D_{21}$ as in Lemma 1.2.3. Clearly $z \in H_{12}$. For any $y \in H_{12}$ we'have 


$$
\begin{aligned}
Q(y, z) & =-\left\langle y^{1}, F(\lambda)_{21} F_{12} u^{2}\right\rangle+\left\langle y^{2}, F_{12} u^{2}\right\rangle-\left\langle D_{21} y^{1}, F_{12} u^{2}\right\rangle \\
& =-\left\langle\lambda I_{21} y^{1}-y^{2}, F_{12} u^{2}\right\rangle .
\end{aligned}
$$

Hence if $y \in N$, then $Q(y, z)=0$. On the other hand

$$
\begin{aligned}
Q(z, z) & =-2 \lambda\left\langle I_{21} F_{12} u^{2}, F_{12} u^{2}\right\rangle+\left\langle D_{21} F_{12} u^{2}, F_{12} u^{2}\right\rangle \\
& \leqq-2 c(\lambda)\left\langle F_{12} u^{2}, u^{2}\right\rangle<0 .
\end{aligned}
$$

Thus $z \notin N$ and consequently $N_{1} \equiv[y+\alpha z ; y \in N]$ contains $N$ properly. Moreover

$$
Q(y+\alpha z, y+\alpha z)=Q(y, y)+|\alpha|^{2} Q(z, z) \leqq 0,
$$

and this shows that $N_{1}$ is a negative subspace, contrary to $N$ being maximal negative.

Conversely, suppose $N$ is a negative subspace with $\Re_{\lambda}(N)=H_{2}$. Lemma 1.3.3 asserts that the mapping $y \rightarrow f^{2}$ of $N$ onto $\Re_{\lambda}(N)$ is one-to-one and that this remains true for any negative subspace containing $N$. Since $N$ already maps onto $\mathrm{H}_{2}$, it follows that $\mathrm{N}$ is maximal negative.

Specializing the above result to the case $F=I$ and dissipative operators we readily obtain another proof of the corollary to Theorem 1.1.1. In fact, we have only to note that if $L$ is dissipative then its graph is negative, and if $\mathfrak{D}(L)$ is dense then any negative subspace containing $\mathfrak{G}(L)$ is the graph of a dissipative extension of $L$.

The foregoing lemmas can be used to connect maximal negative subspaces of $H_{12}$ with maximal dissipative operators on $H_{0}$. To this end we introduce the following

Definition 1.3.3. A transformation $L_{21}$ on $H_{1}$ to $H_{2}$ will be said to engender the operator $L$ on $H_{0}$ to itself if

$$
\begin{aligned}
\mathfrak{D}(L) & =\left[I_{01} y^{1} ; y^{1} \in \mathfrak{D}\left(L_{21}\right) \text { and } L_{21} y^{1} \in I_{20}\left[H_{0}\right]\right], \\
L y^{0} & =I_{20}^{-1} L_{21} I_{01}^{-1} y^{0} .
\end{aligned}
$$

THEOREM 1.3.1. Let $L_{21}$ be a linear transformation on $H_{1}$ to $H_{2}$ whose graph is a maximal negative subspace of $H_{12}$ and let $L$ denote the operator on $H_{0}$ engendered by $L_{21}$. Then $L$ is a closed maximal dissipative operator with dense domain, and the graph of $I_{20} L I_{01}$ is dense in the graph of $L_{21}$.

Proof. According to Lemma 1.3.4, the set $\left[\lambda I_{21} y^{1}-L_{21} y^{1} ; y^{1} \in \mathfrak{D}\left(L_{21}\right)\right]$ fills out $H_{2}$. Further one sees from Definition 1.3.3 that $\lambda I_{21} y^{1}-L_{21} y^{1} \in I_{20}\left[H_{0}\right]$ if and only if $I_{01} y^{1} \in \mathfrak{D}(L)$. Consequently,

$$
\Re(\lambda I-L)=I_{20}^{-1}\left\{\lambda I_{21}-I_{21}\right\} I_{01}^{-1}[\mathfrak{D}(L)]=H_{0} .
$$

On the other hand, setting $y=\left[y^{1}, L_{21} y^{1}\right]$ and $y^{0}=I_{01} y^{1}, y^{0} \in \mathfrak{D}(L)$, we see that 


$$
\begin{aligned}
\left\langle y^{0}, L y^{0}\right\rangle+\left\langle L y^{0}, y^{0}\right\rangle & =\left\langle y^{1}, L_{21} y^{1}\right\rangle+\left\langle L_{21} y^{1}, y^{1}\right\rangle \\
& =Q(y, y)+\left\langle D_{21} y^{1}, y^{1}\right\rangle \leqq 0,
\end{aligned}
$$

so that $L$ is a dissipative operator. Lemma 1.1.1 now implies that $L$ is closed and maximal dissipative and Lemma 1.1 .3 asserts that $\mathfrak{D}(L)$ is dense in $H_{0}$. Next suppose that $y=\left[y^{1}, y^{2}\right] \in(B)\left(L_{21}\right)$, in which case $y^{2}=L_{21} y^{1}$. Then $f^{2}$ $=\lambda I_{21} y^{1}-y^{2} \in H_{2}$ is determined by a Cauchy sequence $\left\{f_{n}^{0}\right\} \subset H_{0}$ such that $f_{n}^{2} \equiv I_{20} f_{n}^{0} \rightarrow f^{2}$. Since $\Re(\lambda I-L)=H_{0}$, there exists a sequence $\left\{u_{n}^{2}\right\} \in \mathfrak{D}(L)$ such that $f_{n}^{0}=\lambda u_{n}^{0}-L u_{n}^{0}$ for all $n$. Setting $u_{n}^{1}=I_{01}^{-1} u_{n}^{0}$, we see that $f_{n}^{2}=\lambda I_{21} u_{n}^{1}-L_{21} u_{n}^{1}$; hence the relation (1.3.7) implies that the sequence $\left\{u_{n}^{1}\right\}$ converges in $H_{1}$ to say $u^{1}$. Thus $\left[u_{n}^{1}, L_{21} u_{n}^{1}=\lambda I_{21} u_{n}^{1}-f_{n}^{2}\right]$ converges in $H_{12}$ to $\left[u^{1}, \lambda I_{21} u^{1}-f^{2}\right]$ and since $L_{21}$ is closed it follows that $u^{1} \in \mathfrak{D}\left(L_{21}\right)$ and $L_{21} u^{1}=\lambda I_{21} u^{1}-f^{2}$. Consequently $w^{1}=u^{1}-y^{1}$ is a solution of $\lambda I_{21} w^{1}-L_{21} w^{1}=\theta$ and (1.3.7) requires that $w^{1}=\theta$. In other words $\left[u_{n}^{1}, L_{21} u_{n}^{1}\right] \rightarrow\left[y^{1}, L_{21} y^{1}\right]$ in $H_{12}$, and since

$$
\left\{u_{n}^{1}\right\} \subset I_{01}^{-1}[\mathfrak{D}(L)]
$$

and $L_{21} u_{n}^{1}=I_{20} L I_{01} u_{n}^{1}$, the last assertion of the theorem has now been verified.

1.4. Duality theory. The present section contains a duality theory for maximal negative and maximal positive subspaces of $H_{12}$. As we shall see, this theory is intimately related to an adjoint theory for maximal dissipative operators. Our central result is

Theorem 1.4.1. Let $N$ be a maximal negative subspace and let $P$ denote its $Q$-orthogonal complement. Then $P$ is maximal positive and $N$ is the $Q$-orthogonal complement of $P$.

We shall prove this theorem with the help of the following two lemmas.

Lemma 1.4.1. Let $N$ be a closed subspace and let $P$ denote its $Q$-orthogonal complement. Then $N$ is again the Q-orthogonal complement of $P$.

Proof. The relation (1.3.6) shows that

$$
Q(y, z)=\left\langle W^{-1} y, z\right\rangle \text {. }
$$

Consequently $P$ is the ordinary orthogonal complement of $W^{-1}[N]$. Since $W$ is a continuous operator $W^{-1}[N]$ is a closed subspace and hence $W^{-1}[N]$ is again the orthogonal complement of $P$. This being so, the relation (1.4.1) now implies that $N$ is the $Q$-orthogonal complement of $P$.

Lemma 1.4.2. If $N$ is a negative and $P$ a positive subspace of $H_{12}$, and if $N$ and $P$ are $Q$-orthogonal complements of one another, then both are maximal.

Proof. It is clear that $N$ and $P$ are both closed linear subspaces of $H_{12}$. Moreover, because of the $U$ isomorphism between positive and negative subspaces, it suffices to show that $N$ is maximal negative. If this were not so, there would be a closed negative subspace, say $N_{1}$, which properly contains 
$N$. Choose $u \in N_{1} \cap N^{\perp}, u \neq \theta$. Then $Q(u, u) \leqq 0$ and $\langle y, u\rangle=0$ for all $y \in N$. According to (1.3.6), $\langle y, u\rangle=Q(y, W u)$ so that $W u \in P$ and therefore $Q(W u, W u) \geqq 0$. We now have

$$
\begin{aligned}
\left\langle u^{1}, u^{2}\right\rangle+\left\langle u^{2}, u^{1}\right\rangle-\left\langle D_{21} u^{1}, u^{1}\right\rangle & =Q(u, u) \leqq 0, \\
\left\langle u^{1}, u^{2}\right\rangle+\left\langle u^{2}, u^{1}\right\rangle+\left\langle D_{21} F_{12} u^{2}, F_{12} u^{2}\right\rangle & =Q(W u, W u) \geqq 0 .
\end{aligned}
$$

As a consequence

$$
-\left\langle D_{21} u^{1}, u^{1}\right\rangle-\left\langle D_{21} F_{12} u^{2}, F_{12} u^{2}\right\rangle \leqq 0
$$

and since $D_{21} \leqq \Theta$, we obtain

$$
\left\langle D_{21} u^{1}, u^{1}\right\rangle=0=\left\langle D_{21} F_{12} u^{2}, F_{12} u^{2}\right\rangle .
$$

Combining this with the inequalities (1.4.2) yields

$$
Q(u, u)=0=Q(W u, W u) .
$$

Now $Q$ is a nonpositive quadratic form on $N_{1}$ and hence the Schwarz inequality (relative to $-Q$ on $N_{1}$ ) implies that $Q(y, u)=0$ for all $y \in N_{1}$. It follows that $u$ belongs to the $Q$-orthogonal complement of $N$, namely $P$. Similarly, $P$ positive, $W u \in P$, and $Q(W u, W u)=0$ imply that $Q(W u, z)=0$ for all $z \in P$; thus $W u \in N$. Consequently $u$ and $W u$ are $Q$-orthogonal so that we obtain from (1.3.6) the result $\langle u, u\rangle=Q(W u, u)=0$. This being contrary to our choice of $u$, we conclude that $N$ is maximal negative.

Proof of Theorem 1.4.1. We are given that $N$ is maximal negative and that $P$ is its $Q$-orthogonal complement. We first show that $P$ is a positive subspace. If this were not so, there would exist a $z \in P$ such that $Q(z, z)<0$. Since $Q(y, z)=0$ for all $y \in N$, we see that $z$ does not belong to $N$ and further that

$$
Q(y+\alpha z, y+\alpha z)=Q(y, y)+|\alpha|^{2} Q(z, z) \leqq 0, \quad y \in N .
$$

It follows that $N_{1} \equiv[y+\alpha z ; y \in N]$ is a negative subspace which contains $N$ properly. This being impossible, we conclude that $P$ is indeed positive. By Lemma 1.4.1, $N$ and $P$ are $Q$-orthogonal complements of one another so that it follows from Lemma 1.4.2 that $P$ is a maximal positive subspace.

Lemma 1.4.3. If $N$ has a dense domain then its $Q$-orthogonal complement is a graph and, conversely, if $N$ is closed graph then its Q-orthogonal complement has a dense domain.

Proof. Let $P$ denote the $Q$-orthogonal complement of $N$. If $P$ has an element of the form $z=\left[\theta, z^{2}\right]$, then $Q(y, z)=\left\langle y^{1}, z^{2}\right\rangle=0$ for all $y \in N$ and, assuming $\mathfrak{D}(N)$ to be dense, this implies $z^{2}=\theta$. It follows that $P$ is a graph if $\mathfrak{D}(N)$ is dense. Suppose next that $N$ is a closed graph but that $\mathfrak{D}(P)$ is not dense in $H_{1}$. Then there is a $u^{2} \in H_{2}, u^{2} \neq \theta$, such that $\left\langle u^{2}, z^{1}\right\rangle=0$ for all $z^{1} \in \mathfrak{D}(P)$. We see as above that $u=\left[\theta, u^{2}\right]$ is $Q$-orthogonal to $P$ and hence by Lemma 1.4.1 
that $u \in N$, contrary to $N$ being a graph. This establishes the second half of the lemma.

A somewhat deeper result in this direction is given by

Lemma 1.4.4. Suppose $N$ is both a maximal negative (maximal positive) subspace and a graph. Then $\mathfrak{D}(N)$ is dense in $H_{1}$ and the $Q$-orthogonal complement of $N$ is also a graph with dense domain.

Proof. Let $P$ denote the $Q$-orthogonal complement of $N$. Since $N$ is maximal negative, $P$ will be positive by Theorem 1.4 .1 and since $N$ is a closed graph, $\mathfrak{D}(P)$ will be dense by the previous lemma. As a consequence, Lemma 1.3.1 asserts that $P$ is also a graph. Applying Lemma 1.4.3 once more we see that $\mathfrak{D}(N)$ is dense.

Incidental to the above development is a new proof of Theorem 1.1.2.

Theorem 1.4.2. If $L$ is a maximal dissipative operator with dense domain, then so is its adjoint operator.

Proof. In this case we suppose $F=I$ so that $H_{1}=H_{0}=H_{2}$. The graph of $L$, say $N$, is by hypothesis negative with dense domain and since $L$ is maximal dissipative, it follows from Lemma 1.3.1 that $N$ is maximal negative. Denoting the $Q$-orthogonal complement of $N$ in $H_{0} \times H_{0}$ by $P$, we see that $P$ is a maximal positive subspace by Theorem 1.4.1. and a graph with dense domain by Lemma 1.4.3. Consequently $U[P]$ is a maximal negative subspace and a graph with dense domain. Now $z \in U[P]$ if and only if

$$
0=Q(y, U z)=-\left\langle y^{1}, z^{2}\right\rangle+\left\langle y^{2}, z^{1}\right\rangle, \quad y^{2}=L y^{1},
$$

for all $y^{1} \in \mathfrak{D}(L)$, that is, if and only if $z^{2}=L^{*} z^{1}$. Thus $U[P]=\left(\mathfrak{S}\left(L^{*}\right)\right.$ and $L^{*}$ is a maximal dissipative operator with dense domain.

Most essential for our purposes is the following

THEOREM 1.4.3. Let $L_{21}$ be a linear transformation on $H_{1}$ to $H_{2}$ whose graph $N$ is a maximal negative subspace of $H_{12}$ and let $P$ be the $Q$-orthogonal complement of $N$. In this case $U[P]$ is also the graph of a linear transformation, say $M_{21}$. Next let $L$ and $M$ denote the operators on $H_{0}$ engendered by $L_{21}$ and $M_{21}$, respectively. Then $L$ and $M$ are maximal dissipative operators, with dense domains, and adjoints of each other.

Proof. Since $N$ is maximal negative and a graph, it follows from Theorem 1.4.1 and Lemma 1.4.4 that $P$ is maximal positive and a graph. Consequently $U[P]$ is maximal negative and a graph. Theorem 1.3.1 therefore implies that $L$ and $M$ are both closed maximal dissipative operators with dense domains. Employing the previous theorem we see that $L^{*}$ is maximal dissipative with dense domain. Now for $y^{0} \in \mathfrak{D}(L), z^{0} \in \mathfrak{D}(M), y^{1}=I_{01}^{-1} y^{0}$, and $z^{1}=I_{01}^{-1} z^{0}$, it is clear that $y=\left[y^{1}, L_{21} y^{1}\right] \in N$ and $z=\left[z^{1}, M_{21} z^{1}\right] \in U[P]$. Thus $U z \in P$ and

$$
\left\langle y^{0}, M z^{0}\right\rangle-\left\langle L y^{0}, z^{0}\right\rangle=\left\langle y^{1}, M_{21} z^{1}\right\rangle-\left\langle L_{21} y^{1}, z^{1}\right\rangle=-Q(y, U z)=0 .
$$


Consequently $M \subset L^{*}$ and since $M$ is itself maximal dissipative, it follows that $M=L^{*}$.

1.5. The boundary space. As we have already mentioned in the introduction, the boundary data assumed by functions in the domain of our differential operator will be represented by a certain space of cosets which we will call the boundary space. We now give an abstract formulation of such a space.

Definition 1.5.1. A closed subspace $N_{0}$ of $H_{12}$ will be called a null space if

$$
Q(y, u)=0
$$$$
\text { for all } y, u \in N_{0} \text {. }
$$

Suppose $N_{0}$ is a null space and let $N_{1}$ denote its Q-orthogonal complement; clearly $N_{0} \subset N_{1}$. The quotient space

$$
H=N_{1} / N_{0}
$$

will be called a boundary space.

It is easy to see that the boundary space $H$ can be made into a hilbert space. In fact, if $\beta$ denotes the projection operator $N_{1} \rightarrow N_{1} \cap N_{0}^{\perp}$, then $\beta y=\beta u$ if and only if $y-u \in N_{0}$. Thus $H$ is algebraically isomorphic with $N_{1} \cap N_{0}^{\perp}$ and the inner product defined on $N_{1} \cap N_{0}^{\perp}$ can be used to implement an inner product in $\boldsymbol{H}$. We shall denote the points of $H$ by $y, z, \cdots$, and we shall indicate that $y \in N_{1}$ belongs to the coset $y \in H$ by writing $\beta y=y$. It will be clear from the context whether $\beta y$ is to be thought of as an element of $H$ or as an element of $N_{1} \cap N_{0}^{\perp}$.

The following lemma gives a simple characterization of $N_{1} \cap N_{0}^{\perp}$.

Lemma 1.5.1. Let $N_{0}$ be a null space and let $N_{1}$ denote its $Q$-orthogonal complement. Then $y \in N_{1} \cap N_{0}^{\perp}$ if and only if both $y$ and Wy belong to $N_{1}$.

Proof. By definition a necessary and sufficient condition for $y \in N_{1} \cap N_{0}^{\perp}$ is that $y$ belong to $N_{1}$ and be orthogonal to $N_{0}$. By (1.3.6) the last half of this condition is equivalent with $W y$ being $Q$-orthogonal to $N_{0}$, in other words, it is equivalent with $W y$ belonging to $N_{1}$.

Lemma 1.5.2. Let $N_{0}$ be a null space and let $N_{1}$ denote its Q-orthogonal complement. If $y, u \in N_{1}$, then

$$
\begin{aligned}
Q(y, u) & =Q(\beta y, \beta u), \\
|Q(\beta y, \beta u)| & \leqq 2\|\beta y\|\|\beta u\|,
\end{aligned}
$$$$
\text { and if } Q(\beta y, \beta u)=0 \text { for all } \beta u \in N_{1} \cap N_{0}^{\perp} \text {, then } \beta y=\theta \text {. }
$$

Proof. It follows from the definition of $N_{1}$ that $Q(y, u)=0$ for all $y \in N_{1}$ and $u \in N_{0}$. Since $y-\beta y$ and $u-\beta u$ both belong to $N_{0}$, we have

$$
Q(y, u)=Q(\{\beta y+[y-\beta y]\},\{\beta u+[u-\beta u]\})=Q(\beta y, \beta u) .
$$

The relation (1.5.4) is an immediate consequence of (1.3.3). Finally if $Q(\beta y, \beta u)=0$ for all $\beta u$, then by (1.5.3), $Q(\beta y, u)=0$ for all $u \in N_{1}$. This means 
that $\beta y$ belongs to the $Q$-orthogonal complement of $N_{1}$ and hence, by Lemma 1.4.1, that $\beta y$ belongs to $N_{0}$. Thus $\beta y$ is orthogonal to itself and therefore $\beta y=\theta$.

It follows that the value of $Q(y, u)$ depends only on the cosets to which $y$ and $u$ belong. This suggests that we introduce the

Definition 1.5.2. We define the form $\boldsymbol{Q}$ on $H$ as

$$
Q(\beta y, \beta u)=Q(\beta y, \beta u) \text {. }
$$

It is clear that $\boldsymbol{Q}$ is a continuous bilinear form and it follows from the preceding lemma that it is nonsingular in the sense that $\boldsymbol{Q}(\boldsymbol{y}, \boldsymbol{u})=0$ for all $\boldsymbol{u} \in \boldsymbol{H}$ implies that $\boldsymbol{y}=\boldsymbol{\theta}$.

Lenma 1.5.3. Let $N_{0}$ be a null space and let $N_{1}$ denote its $Q$-orthogonal complement. The mapping $N=\beta N$ defines a one-to-one correspondence between subspaces of $N_{1}$ which contain $N_{0}$ and subspaces of $H$. This correspondence preserves negativity, positivity, inclusion, and Q-orthogonal subspaces correspond to Q-orthogonal subspaces. In particular, subspaces of $N_{1}$ which are maximal negative (maximal positive) relative to the subspaces of $N_{1}$ contain $N_{0}$ and correspond to maximal negative (maximal positive) subspaces of $\boldsymbol{H}$.

Proof. All the assertions of the lemma save the last, follow directly from properties of the homomorphism $\beta$ established in Lemma 1.5.2. Actually even the last assertion is evident, once it has been shown that a subspace $N$ which is maximal negative, say, relative to the subspaces of $N_{1}$ necessarily contains $N_{0}$. However, if $N$ is negative, so is $N=\beta N$ and $\beta^{-1} N$. Since $\beta^{-1} N$ is a negative subspace of $N_{1}$ containing $N$ and $N_{0}$, it follows from the maximal property that $N$ contains $N_{0}$.

Lemma 1.5.4. Suppose $N_{0}$ is a null space and let $N_{1}$ denote its Q-orthogonal complement. Then any negative (positive) subspace $N$ of $N_{1}$ which is maximal negative (maximal positive) relative to the subspaces of $N_{1}$ is also maximal negative (maximal positive) relative to the subspaces of $H_{12}$. Further if $P$ is the $Q$ orthogonal complement relative to $N_{1}$ of such an $N$, then $P$ is also Q-orthogonal to $N$ relative to $H_{12}$ and maximal positive (maximal negative) relative to the subspaces of $H_{12}$ (and a fortiori maximal relative to the subspaces of $N_{1}$ ).

Proof. Suppose first that $N \subset N_{1}$ is a maximal negative subspace relative to the subspaces of $N_{1}$. It follows from the previous lemma that $N$ consists of cosets of $N_{0}$ and, in particular that $N$ contains $N_{0}$. On the other hand if $N^{\prime}$ is any negative subspace of $H_{12}$ containing $N_{0}$, then since $Q$ is negative on $N^{\prime}$ we obtain as a simple consequence of the Schwarz inequality that each element of $N_{0}$ is $Q$-orthogonal to every element in $N^{\prime}$; in other words $N^{\prime}$ is contained in the $Q$-orthogonal complement of $N_{0}$, namely $N_{1}$. It follows that any negative extension in $H_{12}$ of the given $N$ necessarily lies in $N_{1}$ so that $N$ is also maximal negative relative to the subspaces of $H_{12}$. Likewise we 
note that the $Q$-orthogonal complement of $N$ relative to $H_{12}$ is necessarily a subspace of $N_{1}$ since $N_{1}$ is the $Q$-orthogonal complement of a subspace of $N$, namely $N_{0}$. Thus $P$ is the $Q$-orthogonal complement of a maximal negative subspace and therefore is itself maximal positive by Theorem 1.4.1. Finally, if $N$ had been maximal positive (instead of maximal negative) relative to the subspaces of $N_{1}$, then $U[N]$ would be maximal negative relative to the subspaces of $U\left[N_{1}\right]$. Since $U\left[N_{1}\right]$ is the $Q$-orthogonal complement of the null space $U\left[N_{0}\right]$, the above argument applies and we see that $U[N]$ is maximal negative in $H_{12}$ and hence that $N$ is maximal positive in $H_{12}$. It then follows as above that $P$ is maximal negative in $H_{12}$.

REMARK. If in the above lemma, the null space $N_{0}$ had possessed a dense domain, then the relations

$$
N_{0} \subset N, P \subset N_{1}
$$

imply that $\mathfrak{D}(N), \mathfrak{D}(P)$, and $\mathfrak{D}\left(N_{1}\right)$ are also dense, and hence by Lemmas 1.3.1 and 1.4.3 that $N, P$, and $N_{1}$ are graphs.

Lemma 1.5.5. Let $N$ be a maximal negative (maximal positive) subspace of $H$ and let $\boldsymbol{P}$ be its $\mathbf{Q}$-orthogonal complement. Then $\boldsymbol{P}$ is maximal positive (maximal negative) and $\boldsymbol{N}$ is the $\mathbf{Q}$-orthogonal complement of $P$. In addition, $N=\beta^{-1} \boldsymbol{N}$ and $P=\beta^{-1} P$ are subspaces of $N_{1}$, maximal with respect to $H_{12}$, and $Q$-orthogonal complements.

Proof. Suppose $\boldsymbol{N}$ is, say, maximal negative in $H$. Then according to Lemma 1.5.3, $N=\beta^{-1} N$ will be maximal negative relative to the subspaces of $N_{1}$ and hence, by Lemma 1.5.4, maximal negative relative to the subspaces of $H_{12}$. Lemma 1.5.4 also asserts that the $Q$-orthogonal complement of $N$, say $P$, is maximal positive and a subset of $N_{1}$. It now follows from Lemma 1.5.3 that $P=\beta P$ is maximal positive as well as the $\boldsymbol{Q}$-orthogonal complement of $N$. Likewise, since $N$ is the $Q$-orthogonal complement of $P$ by Theorem 1.4.1, we see that $N$ will be the $Q$-orthogonal complement of $P$.

We now state one of our principal results.

THeorem 1.5.1. Let $N_{0}$ be a null space with dense domain, let $N_{1}$ be its $Q$ orthogonal complement, let $H=N_{1} / N_{0}$. In this case $N_{0}$ and $N_{1}$ will be graphs of linear transformations, say, $L_{21}^{0}$ and $L_{21}^{1}$, respectively, which engender the operators $L_{0}$ and $L_{1}$, respectively. Suppose, in addition, that $\mathfrak{D}\left(I_{20} L_{0} I_{01}\right)$ is dense in $H_{1}$. Then there is a one-to-one correspondence between the maximal negative subspaces $[\boldsymbol{N}]$ of $\boldsymbol{H}$, taken with respect to the form $\boldsymbol{Q}$, and the maximal dissipative operators $[L]$ such that $L_{0} \subset L \subset L_{1}$, this correspondence being defined by

$$
\mathfrak{D}(L)=\left[y^{0} ; \beta\left[I_{01}^{-1} y^{0}, I_{20} L_{1} y^{0}\right] \in N\right],
$$

which is dense in $H_{0}$.

The adjoint operator $M=L^{*}$ is again maximal dissipative with dense domain 
and can be described as follows: $U\left[N_{0}\right]$ and $U\left[N_{1}\right]$ are graphs of linear transformations, say, $M_{21}^{0}$ and $M_{21}^{1}$, respectively, which engender the operators $M_{0}$ and $M_{1}$, respectively. Let $\boldsymbol{P}$ be the $\mathbf{Q}$-orthogonal complement of $\boldsymbol{N}$. Then $\boldsymbol{P}$ is maximal positive, $M_{0} \subset M \subset M_{1}$, and

$$
\mathfrak{D}(M)=\left[z^{0} ; \beta U\left[I_{01}^{-1} z^{0}, I_{20} M_{1} z^{0}\right] \in P\right] .
$$

Proof. According to Lemma 1.5.3 there is a one-to-one correspondence between the maximal negative subspaces $[N]$ of $H$ and the subspaces of $N_{1}$ which are maximal negative relative to the subspaces of $N_{1}$, this correspondence being given by $N=\beta^{-1} N$. By Lemma 1.5.4 each such $N$ is maximal negative in $H_{12}$ and a graph, say of $L_{21}$. Theorem 1.3.1 now asserts that $L_{21}$ engenders a maximal dissipative operator $L$ with dense domain and that the graph of $I_{20} L I_{01}$ is dense in $N$. It follows that each $L$ obtained in this way can correspond to only one maximal negative subspace in $N_{1}$ and hence to only one maximal negative subspace of $H$. Since $L_{21}^{0} \subset L_{21} \subset L_{21}^{1}$, it is further clear that $L_{0} \subset L \subset L_{1}$. Finally, $L$ is a restriction of $L_{1}$ and hence is completely determined once its domain is given. By Definition 1.3.3

$$
\mathfrak{D}(L)=\left[y^{0} ;\left[I_{01}^{-1} y^{0}, I_{20} L_{1} y^{0}\right] \in N\right],
$$

or, equivalently, $\mathfrak{D}(L)$ can be given by (1.5.6).

The adjoint of $L$ is described in Theorem 1.4.3. If $P$ is the $Q$-orthogonal complement of $N$ and if $M_{21}$ is the transformation whose graph is $U[P]$, then $M=L^{*}$ is engendered by $M_{21}$. It also follows from Theorem 1.4.3 that $M$ is maximal dissipative and has a dense domain. According to Lemma 1.5.4, $N_{0} \subset P \subset N_{1}$ so that $M_{21}^{0} \subset M_{21} \subset M_{21}^{1}$ and hence $M_{0} \subset M \subset M_{1}$. In particular, then $M$ is a restriction of $M_{1}$ with domain

$$
\mathfrak{D}(M)=\left[z^{0} ; U\left[I_{01}^{-1} z^{0}, I_{20} M_{1} z^{0}\right] \in P\right] .
$$

With $P$ denoting the $Q$-orthogonal complement of $N$, we see by Lemma 1.5 .5 that $P$ is maximal positive and that $P=\beta^{-1} P$. Consequently, $\mathfrak{D}(M)$ may also be described as in (1.5.7).

It remains to show that any maximal dissipative operator $L^{\prime}$, such that $L_{0} \subset L^{\prime} \subset L_{1}$, corresponds as above to some maximal negative subspace $N$ of $H$. Suppose, therefore, that $L^{\prime}$ is such an operator and set $L_{21}^{\prime}=I_{20} L^{\prime} I_{01}$ and $N^{\prime}=\mathfrak{S}\left(L_{21}^{\prime}\right)$. By assumption $L_{21}^{\prime} \supset I_{20} L_{0} I_{01}$. We now show that $N^{\prime}$ is a negative subspace of $H_{12}$. In the first place, since $L^{\prime}$ is dissipative,

$$
\left\langle L_{21}^{\prime} y^{1}, y^{1}\right\rangle+\left\langle y^{1}, L_{21}^{\prime} y^{1}\right\rangle=\left\langle L^{\prime} I_{01} y^{1}, I_{01} y^{1}\right\rangle+\left\langle I_{01} y^{1}, L^{\prime} I_{01} y^{1}\right\rangle \leqq 0
$$

for all $y^{1} \in \mathfrak{D}\left(L_{21}^{\prime}\right)$. Suppose then that $Q(y, y)>0$ for some $y \in N^{\prime}$. Choosing a sequence $\left\{u_{n}^{1}\right\}$ in $\mathfrak{D}\left(I_{20} L_{0} I_{01}\right)$ with the property that $u_{n}^{1} \rightarrow y^{1}$ and setting 
$u_{n}=\left[u_{n}^{1}, L_{21}^{\prime} u_{n}^{1}\right]$, we see that $u_{n} \in N_{0}$ and hence that

$0<Q(y, y)=Q\left(y-u_{n}, y-u_{n}\right)$

$$
\begin{aligned}
= & \left\langle\left(y^{1}-u_{n}^{1}\right), L_{21}^{\prime}\left(y^{1}-u_{n}^{1}\right)\right\rangle+\left\langle L_{21}^{\prime}\left(y^{1}-u_{n}^{1}\right),\left(y^{1}-u_{n}^{1}\right)\right\rangle \\
& -\left\langle D_{21}\left(y^{1}-u_{n}^{1}\right),\left(y^{1}-u_{n}^{1}\right)\right\rangle \leqq-\left\langle D_{21}\left(y^{1}-u_{n}^{1}\right),\left(y^{1}-u_{n}^{1}\right)\right\rangle \\
\leqq & \left\langle F_{21}\left(y^{1}-u_{n}^{1}\right),\left(y^{1}-u_{n}^{1}\right)\right\rangle=\left\|y^{1}-u_{n}^{1}\right\|_{1}^{2} .
\end{aligned}
$$

However, this is impossible since the last term tends to zero as $n \rightarrow \infty$. It follows that $N^{\prime}$ is a negative subspace, obviously contained in $N_{1}$. There therefore exists a subspace $N \subset N_{1}$, maximal negative with respect to all subspaces of $N_{1}$ which contains $N^{\prime}$. Now $N$ is the graph of a transformation $L_{21}$ and as in the first part of the proof, $L_{21}$ engenders a maximal dissipative operator $L \subset L_{1}$ which corresponds to the maximal negative subspace $N=\beta N$ as in (1.5.6). It is clear from the construction that $L^{\prime} \subset L$ and since $L^{\prime}$ is maximal dissipative we conclude that $L^{\prime}=L$. In other words, $L^{\prime}$ corresponds as above to the maximal negative subspace $N$.

COROLlaRY. Theorem 1.5.1 characterizes all maximal and properly dissipative restrictions of $L_{1}$, that is, all maximal restrictions such that $Q(y, y) \leqq 0$ for each $y=\left[I_{01}^{-1} y^{0}, I_{20} L y^{0}\right]$ with $y^{0} \in \mathfrak{D}(L)$.

Proof. It is clear that if $L$ is characterized as in (1.5.6), then $Q(y, y) \leqq 0$ for all $y=\left[I_{01}^{-1} y^{0}, I_{20} L y^{0}\right]$ with $y^{0} \in \mathfrak{D}(L)$. Conversely, suppose that $L$ is a maximal and properly dissipative restriction of $L_{1}$. Then $\beta\left[(5)\left(I_{20} L I_{01}^{-1}\right)\right]$ is a negative subspace of $\boldsymbol{H}$ and hence contained in some maximal negative subspace, say $N^{\prime}$. Let $L^{\prime} \subset L_{1}$ be defined by (1.5.6) with $N$ replaced by $N^{\prime}$. Then clearly $L \subset L^{\prime}$ and since both $L$ and $L^{\prime}$ are maximal dissipative it follows that $L=L^{\prime}$, which was to be proved.

The following remarks are of interest in connection with the boundary space for $L_{1}$. According to Lemma 1.5.1, the elements of $N_{1} \cap N_{0}^{\perp}$ are just the

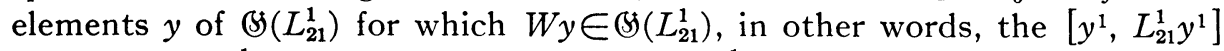
such that $F_{12} L_{21}^{1} y^{1}$ as well as $y^{1}$ belong to $\mathfrak{D}\left(L_{21}^{1}\right)$ and for which

$$
L_{21}^{1}\left[F_{12} L_{21}^{1} y^{1}\right]=F_{21} y^{1}+D_{21} F_{12} L_{21}^{1} y^{1} .
$$

Since $\mathfrak{D}\left(M_{21}^{1}\right)=\mathfrak{D}\left(L_{21}^{1}\right)$ and $M_{21}^{1}=-L_{21}^{1}+D_{21}$, the above relation can be rewritten as

$$
M_{21}^{1} F_{12} L_{21}^{1} y^{1}=-F_{21} y^{1} .
$$

Thus we can represent $H$ as the subspace of $\$\left(L_{21}^{1}\right)$ which corresponds to the solution space of (1.5.8). We shall not pursue this matter any further; neverthe-less it should be remarked that this is clearly a starting point for an investigation of the boundary space of $L_{1}$. 
The next two lemmas deal with properties of $L_{0}$ and $L_{1}$. For one spatial variable it was shown in [12] that both $L_{0}$ and $L_{1}$ are closed; in the many variable case $L_{0}$ is closed, but it seems doubtful that $L_{1}$ is closed. Our best result in this direction is the following.

Lemma 1.5.6. Let $L_{21}$ be a closed transformation on $H_{1}$ to $H_{2}$ whose graph is negative and let $L$ denote the operator engendered by $L_{21}$. Then $L$ is closed.

Proof. Suppose $\left[y_{n}^{0}, L y_{n}^{0}\right] \rightarrow\left[y^{0}, g^{0}\right]$ in $H_{0} \times H_{0}$ and set $y_{n}^{1}=I_{01}^{-1} y_{n}^{0}$ and $f_{n}^{2}=I_{21} y_{n}^{1}-L_{21} y_{n}^{1}$. Then $I_{21} y_{n}^{1}=I_{20} y_{n}^{0} \rightarrow I_{20} y^{0}, L_{21} y_{n}^{1}=I_{20} L y_{n}^{0} \rightarrow I_{20} g^{0}$, and $f_{n}^{2} \rightarrow I_{20} y^{0}$ $-I_{20} g^{0}$. Consequently Lemma 1.3.2 applies and we see that $\left\{y_{n}^{1}\right\}$ is a Cauchy sequence in $H_{1}$ converging to some element, say $y^{1}$. Since $I_{01}$ is continuous it follows that $I_{01} y^{1}=y^{0}$. Thus $\left[y_{n}^{1}, L_{21} y_{n}^{1}\right] \rightarrow\left[y^{1}, I_{20} g^{0}\right]$ in $H_{12}$ and, $L_{21}$ being closed, we see that $y^{1} \in \mathfrak{D}\left(L_{21}\right)$ and that $L_{21} y^{1}=I_{20} g^{0}$. According to Definition 1.3.3, $y^{0}=I_{01} y^{1} \in \mathfrak{D}(L)$ and $L y^{0}=I_{20}^{-1} L_{21} y^{1}=g^{0}$; the operator $L$ is therefore closed.

Lemma 1.5.7. In the notation of Theorem 1.5.1, $\mathfrak{D}\left(L_{0}\right)$ is dense in $H_{0}$ and $M_{1} \subset L_{0}^{*}$.

Proof. According to Lemma 1.2.1, $I_{01}$ is a continuous map of $H_{1}$ into $H_{0}$ and it is clear from this construction that $\Re\left(I_{01}\right)$ contains $\mathfrak{D}(F)$. Since $\mathfrak{D}\left(I_{20} L_{0} I_{01}\right)$ is dense in $H_{1}$, it follows that $\mathfrak{D}\left(L_{0}\right)=I_{01}\left[\mathfrak{D}\left(I_{20} L_{0} I_{01}\right)\right]$ is dense in $\mathfrak{D}(F)^{-}=H_{0}$. On the other hand for $y^{0} \in \mathfrak{D}\left(L_{0}\right), z^{0} \in \mathfrak{D}\left(M_{1}\right), y^{1}=I_{01}^{-1} y^{0}$, and $z^{1}=I_{01}^{-1} z^{0}$, we have

$$
\begin{aligned}
\left\langle L_{0} y^{0}, z^{0}\right\rangle-\left\langle y^{0}, M_{1} z^{0}\right\rangle & =\left\langle L_{21}^{0} y^{1}, z^{1}\right\rangle-\left\langle y^{1}, M_{21}^{1} z^{1}\right\rangle \\
& =Q\left(\left[y^{1}, L_{21}^{0} y^{1}\right],\left[z^{1}, L_{21}^{1} z^{1}\right]\right)=0
\end{aligned}
$$

so that $M_{1} \subset L_{0}^{*}$.

LeMma 1.5.8. Let $N_{0}$ be a null space and the graph of a linear transformation, say $L_{21}^{0}$, which engenders the operator $L_{0}$. Suppose, in addition, that $\mathfrak{D}\left(I_{20} L_{0} I_{01}\right)$ is a dense in $H_{1}$. Then $L_{0}$ is conservative if and only if $I=F$.

Proof. For $y^{0} \in \mathfrak{D}\left(L_{0}\right)$ and $y^{1}=I_{01}^{-1} y^{0}$ we have

$$
\left\langle L_{0} y^{0}, y^{0}\right\rangle+\left\langle y^{0}, L_{0} y^{0}\right\rangle=\left\langle D_{21} y^{1}, y^{1}\right\rangle .
$$

If $L_{0}$ is conservative, then $\left\langle D_{21} y^{1}, y^{1}\right\rangle=0$ for all $y^{1} \in \mathfrak{D}\left(I_{20} L_{0} I_{01}\right)$, which is assumed to be dense in $H_{1}$. Thus this relation holds for all $y^{1} \in H_{1}$; replacing $y^{1}$ by $u^{1}+\epsilon v^{1}, \epsilon=1, i$, in turn and adding, we obtain $\left\langle D_{12} u^{1}, v^{1}\right\rangle=0$ for all $u^{1}, v^{1} \in H_{1}$, from which it follows that $D_{21}=\Theta$. Now for $y^{0} \in \mathfrak{D}(F)$, it is readily seen that

$$
(I-F) y^{0}=I_{20}^{-1} D_{21} I_{01}^{-1} y^{0}=\theta
$$


so that $F=I$. Conversely, if $F=I$ then the relation (1.5.9) shows that $L_{0}$ is conservative.

Now if $F=I$ we have $H_{1}=H_{0}=H_{2}$ and there is no longer any distinction between an operator $L_{21}$ and the operator $L$ which it engenders. Thus, in this case, the operators $L_{0}$ and $L_{1}$ of Theorem 1.5.1 are closed and it follows from $Q(y, z)=\left\langle y^{1}, z^{2}\right\rangle+\left\langle y^{2}, z^{1}\right\rangle$ that $L_{1}=-L_{0}^{*}$. If $L$ is a conservative extension of $L_{0}$, then

$$
L_{1}=-L_{0}^{*} \supset-L^{*} \supset L
$$

whereas if $i L$ is a self-adjoint restriction of $i L_{1}$, then

$$
L_{0}=-L_{1}^{*} C-L^{*}=L .
$$

In either case $L_{0} \subset L \subset L_{1}$. Conditions that $L \supset L_{0}$ be a conservative maximal dissipative operator have been given by J. W. Calkin [1, Theorems 2.9 and 4.1]. For the sake of completeness we now give another version of this result which is slightly more general in that it includes all maximal dissipative extensions of $L_{0}$. In view of Lemma 1.5.8, we may, without loss of generality, assume that $F=I$.

Theorem 1.5.2. Suppose $F=I$ and let $L_{0}$ be a closed conservative operator with dense domain. Set $L_{1}=-L_{0}^{*}$ and $\boldsymbol{H}=\circlearrowleft\left(L_{0}\right) / \$\left(L_{1}\right)$. Then there is a one-toone correspondence between the maximal negative subspaces $[N]$ of $\boldsymbol{H}$, taken with respect to the form $Q$, and the maximal dissipative operators $[L]$ such that $L_{0} \subset L$, this correspondence being defined by the fact that $L$ is necessarily a restriction of $L_{1}$ and

$$
\mathfrak{D}(L)=\left[y^{0} ; \beta\left[y^{0}, L_{1} y^{0}\right] \in N\right] .
$$

Moreover, $L$ will be conservative if and only if $\boldsymbol{N}$ is a null subspace of $\boldsymbol{H}$, whereas iL will be self-adjoint if and only if $N=P$, where $P$ is the Q-orthogonal complement of the maximal negative subspace $N$.

Proof. Since $F=I$ it is easy to see that $N_{0}=\circlearrowleft\left(L_{0}\right)$ is a null space with dense domain and that $N_{1}=\mathscr{S}\left(L_{1}\right)$ is its $Q$-orthogonal complement. According to Lemma 1.1.5 each maximal dissipative extension of $L_{0}$ is also a restriction of $L_{1}$. Consequently the first assertion of the theorem is a special case of Theorem 1.5.1. For $y=\left[y^{0}, L_{1} y^{0}\right]$

$$
\left\langle L_{1} y^{0}, y^{0}\right\rangle+\left\langle y^{0}, L_{1} y^{0}\right\rangle=Q(y, y)=Q(\beta y, \beta y) .
$$

Therefore, if $L$ is conservative, then $Q(y, y)=0$ for all $y \in N$. Replacing $y$ by $u+\epsilon v, \epsilon=1, i$, in turn, and adding, we obtain $\boldsymbol{Q}(\boldsymbol{u}, \boldsymbol{v})=0$ for all $u, v \in N$; hence $N$ is a null space. Conversely, if $N$ is a null space, then (1.5.10) shows that $L$ is conservative. On the other hand, Lemma 1.1.4 asserts that $i L$ is self-adjoint if and only if $L$ and $M=L^{*}$ are conservative. The above argument shows that if $L$ is conservative that $N$ is a null space. Further, if $P$ denotes the 
$Q$-orthogonally complement of $\$(L)$, then $\$(M)=U[P]$, as in the proof of Theorem 1.5.1. For $z=\left[z^{0}, M z^{0}\right] \in U[P]$ we have

$$
\left\langle M z^{0}, z^{0}\right\rangle+\left\langle z^{0}, M z^{0}\right\rangle=Q(U z, U z)=Q(\beta U z, \beta U z) .
$$

Since $U$ maps $U[P]$ in a one-to-one fashion on $P$, we see as above that $M$ being conservative is equivalent with $P$ and hence $P=\beta P$ being null. Finally if $N$ and $P$ are null, then each is contained in its $Q$-orthogonal complement, namely $P$ and $N$, respectively; consequently $N=P$. Conversely, if $N=P$ then this subspace is both positive and negative, and hence it is a null space. The relations (1.5.10) and (1.5.11) then imply that $L$ and $M$ are conservative, and thus that $i L$ is self-adjoint.

It will be observed that there is a lack of symmetry between the expression (1.5.6) for $\mathfrak{D}(L)$ and (1.5.7) for $\mathfrak{D}(M)$. This is a consequence of the fact that both domains are delimited by means of the boundary space for $L$, whereas symmetry requires that $\mathfrak{D}(M)$ be delimited in terms of the boundary space for $M$. The purpose of the next section is to rectify this situation.

1.6. The dual boundary space. Throughout this section $N_{0}$ will be a fixed null space with $Q$-orthogonal complement $N_{1}$ and $H=N_{1} / N_{0}$ will denote the associated boundary space. It is clear that $N_{0}^{\prime}=U\left[N_{0}\right]$ is also a null space and the relation $Q(U y, U z)=-Q(y, z)$ shows that $N_{1}^{\prime}=U\left[N_{1}\right]$ is the $Q$ orthogonal complement of $N_{0}^{\prime}$. Thus

$$
H^{\prime}=N_{1}^{\prime} / N_{0}^{\prime}
$$

is again a boundary space, and we refer to this space as the dual boundary space. We proceed to study the relation between these two boundary spaces.

As before $H^{\prime}$ is isomorphic with $N_{1}^{\prime} \cap N_{0}^{\prime \perp}$ and we shall denote the $Q$ induced bilinear form on $H^{\prime}$ by $Q^{\prime}$. Likewise, we shall let $\beta^{\prime}$ denote both the natural homomorphism of $N_{1}^{\prime}$ onto $H^{\prime}$ and the projection operator of $N_{1}^{\prime}$ onto $N_{1}^{\prime} \cap N_{0}^{\prime \perp}$. Given an operator $T$ on $H_{12}$ we shall symbolize its restriction on $N_{1} \cap N_{0}^{\perp}$ by $T_{0}$ and its restriction on $N_{1}^{\prime} \cap N_{0}^{\prime \perp}$ by $T_{0}^{\prime}$.

In view of the way in which $\boldsymbol{H}^{\prime}$ has been defined, one should expect the operator $U$ to provide the principal link between $H$ and $H^{\prime}$. The following lemma shows that this is the case.

Lemma 1.6.1. The transformation $\beta^{\prime} U_{0}$ is one-to-one and bicontinuous on $N_{1} \cap N_{0}^{\perp}$ to $N_{1}^{\prime} \cap N_{0}^{\prime \perp}$ with inverse $\beta U_{0}^{\prime}$. Moreover

$$
Q\left(\beta^{\prime} U_{0} y, \beta^{\prime} U_{0} z\right)=-Q(y, z), \quad y, z \in N_{1} \cap N_{0}^{\perp} .
$$

Proof. Given $z \in N_{1}^{\prime} \cap N_{0}^{\prime \perp} \subset U\left[N_{1}\right]$, there exists a $y \in N_{1} \cap N_{0}^{\perp}$ and a $u \in N_{0}$ such that $z=U y+U u$. Since $U u \in N_{0}^{\prime}$, we see that $z=\beta^{\prime} z=\beta^{\prime} U y$; this shows that $\beta^{\prime} U_{0}$ is onto $N_{1}^{\prime} \cap N_{0}^{\prime \perp}$. On the other hand, given $y \in N_{1} \cap N_{0}^{\perp}$, set $z=\beta^{\prime} U_{0} y$ and $v=z-U y$. Then $\beta^{\prime} v=z-z=\theta$ from which it follows that $v \in N_{0}^{\prime}$. Consequently $U v=U_{0}^{\prime} z-y \in N_{0}$ and $y=\beta y=\beta U_{0}^{\prime} z$; in other words $\left(\beta U_{0}^{\prime}\right)\left(\beta^{\prime} U_{0}\right)$ 
$=I_{0}$. Since $\beta^{\prime} U_{0}$ was onto, we infer that $\beta U_{0}^{\prime}=\left(\beta^{\prime} U_{0}\right)^{-1}$ and hence that $\beta^{\prime} U_{0}$ is one-to-one and bicontinuous. Finally the relation (1.6.2) follows from (1.3.6) and (1.5.3).

Lemma 1.6.2. $V_{0}$ is a unitary map on $N_{1} \cap N_{0}^{\perp}$ to $N_{1}^{\prime} \cap N_{0}^{\prime \perp}$ with $V_{0}^{-1}=-V_{0}^{\prime}$.

Proof. It is clear from (1.3.4) that $V$ is a unitary map on $H_{12}$ to itself with $V^{-1}=-V$. It therefore suffices to show that $V_{0}$ maps $N_{1} \cap N_{0}^{\perp}$ onto $N_{1}^{\prime} \cap N_{0}^{\prime \perp}$. To this end let $y \in N_{1} \cap N_{0}^{\perp}$ and suppose $u$ is an arbitrary element of $N_{0}$. Then by (1.3.6)

$$
0=\langle y, u\rangle=Q(U V y, u)=-Q(V y, U u) ;
$$

since $N_{0}^{\prime}=U\left[N_{0}\right]$ it follows that $V_{0} y$ belongs to the $Q$-orthogonal complement of $N_{0}^{\prime}$, that is to $N_{1}^{\prime}$. Further

$$
\langle V y, U u\rangle=Q(W V y, U u)=Q(U V V y, U u)=-Q(V V y, u)=Q(y, u)=0
$$

so that $V_{0} y$ is orthogonal to $N_{0}^{\prime}$. Together these results imply that $V_{0} y$ $\in N_{1}^{\prime} \cap N_{0}^{\prime \perp}$. Similarly, it can be shown that $V_{0}^{\prime}$ maps $N_{1}^{\prime} \cap N_{0}^{\prime \perp}$ into $N_{1} \cap N_{0}^{\perp}$. On the other hand $V^{2}=-I$ implies $V_{0} V_{0}^{\prime}=-I_{0}^{\prime}$, and this shows that the map is onto.

Lemma 1.6.3. $\beta W_{0}$ is symmetric, one-to-one, and bicontinuous on $N_{1} \cap N_{0}^{\perp}$ to itself with $\left(\beta W_{0}\right)^{-1}=-V_{0}^{\prime} \beta^{\prime} U_{0}=\beta_{1}\left(W^{-1}\right)_{0}$. Similarly $\beta^{\prime} W_{0}^{\prime}$ is symmetric, one-to-one, bicontinuous on $N_{1}^{\prime} \cap N_{0}^{\prime \perp}$ to itself with $\left(\beta^{\prime} W_{0}^{\prime}\right)^{-1}=-V_{0} \beta U_{0}^{\prime}$ $=\beta_{1}^{\prime}\left(W^{-1}\right)_{0}^{\prime}$. Here $\beta_{1}$ and $\beta_{1}^{\prime}$ are projections on $H_{12}$ to $N_{1} \cap N_{0}^{\perp}$ and $N_{1}^{\prime} \cap N_{0}^{\prime \perp}$, respectively.

Proof. Since $W=U V$, it is an immediate consequence of Lemmas 1.6.1 and 1.6.2 that $\beta W_{0}=\left(\beta U_{0}^{\prime}\right) V_{0}$ is one-to-one and bicontinuous on $N_{1} \cap N_{0}^{\perp}$ to itself. Further $\beta W_{0}\left(V_{0}^{\prime} \beta^{\prime} U_{0}\right)=\beta U_{0}^{\prime} V_{0} V_{0}^{\prime} \beta^{\prime} U_{0}=-I_{0}$ so that $\left(\beta W_{0}\right)^{-1}$ $=-V_{0}^{\prime} \beta^{\prime} U_{0}$. Now for $y \in N_{1} \cap N_{0}^{\perp},\left(I-\beta^{\prime}\right) U_{0} y \in N_{0}^{\prime}$ and since $V$ is unitary and maps $N_{1}^{\prime} \cap N_{0}^{\prime \perp}$ onto $N_{1} \cap N_{0}^{\perp}$, we see that $V\left(I-\beta^{\prime}\right) U_{0} y \in\left(N_{1} \cap N_{0}^{\perp}\right)^{\perp}$. Hence $\beta_{1} V\left(I-\beta^{\prime}\right) U_{0} y=\theta$ and $-\beta_{1}\left(W^{-1}\right)_{0}=\beta_{1} V U_{0}=\beta_{1} V \beta^{\prime} U_{0}=V_{0}^{\prime} \beta^{\prime} U_{0}$. Finally we see from (1.3.6) that for $y, z \in N_{1} \cap N_{0}^{\perp}$ we have

$$
\left\langle\beta W_{0} y, z\right\rangle=\langle W y, z\rangle=Q\left(W^{2} y, z\right)=Q\left(y, W^{2} z\right)=\langle y, W z\rangle=\left\langle y, \beta W_{0} z\right\rangle
$$

and hence $\beta W_{0}$ is symmetric. The results for $\beta^{\prime} W_{0}^{\prime}$ follow in a similar fashion.

Corollary. For $y, z \in N_{1} \cap N_{0}^{\perp}$ we have

$$
\left\langle\left(\beta W_{0}\right)^{-1} y, z\right\rangle=Q(y, z)=\left\langle y,\left(\beta W_{0}\right)^{-1} z\right\rangle .
$$

Proof. It suffices to note that for $y, z \in N_{1} \cap N_{0}^{\perp}$

$$
Q(y, z)=\left\langle W^{-1} y, z\right\rangle=\left\langle\beta_{1}\left(W^{-1}\right)_{0} y, z\right\rangle=\left\langle\left(\beta W_{0}\right)^{-1} y, z\right\rangle .
$$

It is clear that the transformations $\beta^{\prime} U_{0}, \beta U_{0}^{\prime}, \beta W_{0}$, and $\beta^{\prime} W_{0}^{\prime}$ induce corresponding transformations on $H$ and $H^{\prime}$ which we denote by $U, U^{\prime}, W$, 
and $W^{\prime}$, respectively. These transformations are all one-to-one and bicontinuous, $U^{\prime}=U^{-1}, \boldsymbol{Q}^{\prime}\left(U_{y}, U_{z}\right)=-\boldsymbol{Q}(\boldsymbol{y}, \boldsymbol{z})$, and $W$ and $W^{\prime}$ are symmetric with

$$
\begin{aligned}
\boldsymbol{Q}(W y, z) & =\langle y, z\rangle=\boldsymbol{Q}(y, W z), \\
\boldsymbol{Q}^{\prime}\left(W^{\prime} y^{\prime}, z^{\prime}\right) & =\left\langle y^{\prime}, z^{\prime}\right\rangle=\boldsymbol{Q}^{\prime}\left(y^{\prime}, W^{\prime} z^{\prime}\right) .
\end{aligned}
$$

It readily follows that negative (maximal negative, positive, maximal positive) subspaces of $H$ correspond under the $U$ mapping to positive (maximal positive, negative, maximal negative) subspaces of $\boldsymbol{H}^{\prime}$.

Returning to Theorem 1.5.1, we can now define $\mathfrak{D}(M)$ in a manner completely analogous with $\mathfrak{D}(L)$.

THEOREM 1.6.1. In the notation of Theorem 1.5.1., we have

$$
\mathfrak{D}(M)=\left[z^{0} ; \beta^{\prime}\left[I_{01}^{-1} z^{0}, I_{20} M_{1} z^{0}\right] \in U[P]\right],
$$

and $U[P]$ is a maximal negative subspace of $H^{\prime}$.

Proof. As was noted above, $P$ being maximal positive in $H$ implies that $\boldsymbol{U}[\boldsymbol{P}]$ is maximal negative in $\boldsymbol{H}^{\prime}$. Further, each $z \in N_{1}^{\prime}$ has the representation $z=\beta^{\prime} z+v$ where $v \in N_{0}^{\prime}$. Thus $U z=U_{0}^{\prime} \beta^{\prime} z+U v$ and since $U v \in N_{0}$, we have $\beta U z=\beta U_{0}^{\prime} \beta^{\prime} z$. Consequently $\beta U z \in P$ if and only if $\beta U_{0}^{\prime} \beta^{\prime} z \in P$ and hence by Lemma 1.6.1, if and only if $\beta^{\prime} z=\beta^{\prime} U_{0}\left(\beta U_{0}^{\prime} \beta^{\prime} z\right)$ belongs to $\beta^{\prime} U_{0}[P]=U[P]$. The relation (1.6.5) now follows directly from (1.5.7).

The previous result can also be used to characterize the negative and maximal negative subspaces of $H$. In fact, $W^{-1}$ being symmetric, we see that $H$ splits into two orthogonal manifolds $H^{+}$and $H^{-}, H=H^{+} \oplus H^{-}$; these are respectively the positive and negative eigenspaces of $W^{-1}$. If we define

$$
\begin{array}{ll}
\langle y, z\rangle_{+}=\left\langle W^{-1} y, z\right\rangle & \text { for } y, z \in H^{+}, \\
\langle y, z\rangle_{-}=\left\langle W^{-1} y, z\right\rangle & \text { for } y, z \in H^{-},
\end{array}
$$

then $\mathrm{H}^{+}$and $H^{-}$are again hilbert spaces. In view of the fact that $W$ and its inverse are bounded operators, the new topologies are equivalent with the original $H$ topologies for each subspace. Suppose next that $N$ is a negative subspace of $H$ and for $y \in N$ set $y=y^{+}+y^{-}$, where $y^{+} \in H^{+}$and $y^{-} \in H^{-}$. Then

$$
\boldsymbol{Q}(\boldsymbol{y}, \boldsymbol{y})=\left\langle W^{-1} \boldsymbol{y}, \boldsymbol{y}\right\rangle=\left\|\boldsymbol{y}^{+}\right\|_{+}^{2}-\left\|\boldsymbol{y}^{-}\right\|_{-}^{2} \leqq 0 .
$$

As a consequence of this inequality $J y^{-} \equiv y^{+}$defines a contraction transformation on $\mathrm{H}^{-}$to $\mathrm{H}^{+}$with

$$
N=\left[y^{-}+J y^{-} ; y^{-} \in \mathfrak{D}(J)\right] .
$$

Conversely if $J$ is a contraction transformation of this kind, then the set of vectors $\left[y^{-}+J y^{-} ; y^{-} \in \mathfrak{D}(J)\right]$ is a negative subspace of $H$. We have therefore defined an inclusion preserving correspondence between negative sub- 
spaces of $H$ and contraction transformations on $H^{-}$to $H^{+}$. It is clear that $N$ will be maximal negative if and only if the corresponding contraction transformation is defined on all of $\mathrm{H}^{-}$.

\section{Chapter II. Dissipative hyperbolic systems}

2.1. Properly dissipative generators of the basic system. We return now to the dissipative hyperbolic system described in the introduction. By applying the foregoing theory we shall obtain a characterization of the properly dissipative generators first of the system (1.2) and later of this system coupled at the boundary to an auxiliary dissipative system. In the appendix to this chapter we shall construct a class of maximal dissipative extensions of our minimal operator and the dual class of maximal dissipative restrictions of our maximal operator, neither class being generators of properly dissipative solutions.

As remarked in the introduction, energy considerations require that the basic hilbert space be $H_{0}=L_{2}(\Delta ; E)$ and the desire to work with a finite boundary integral motivates our use of the auxiliary hilbert spaces $H_{1}$ and $H_{2}$. We construct $H_{1}$ and $H_{2}$ as in $\S 1.2$ by means of the self-adjoint operator $F$ defined as

$$
F y^{0}=F(\cdot) y^{0}(\cdot)
$$

where $F(\chi)=I-D(\chi)$ and $D(\chi) \leqq \Theta$ for all $\chi \in \Delta$;

$$
\mathfrak{D}(F)=\left[y^{0} ; y^{0}(\cdot) \text { and } F(\cdot) y^{0}(\cdot) \in L_{2}(\Delta ; E)\right] .
$$

It is readily verified that $F$ is self-adjoint and satisfies the condition (1.2.1). Since the functions in $\mathfrak{D}(F)$ are dense in $L_{2}(\Delta ; E F)$ and those in $\Re(F)$ $=L_{2}(\Delta ; E)$ are dense in $L_{2}\left(\Delta ; E F^{-1}\right)$, we see at once that $H_{1}$ and $H_{2}$ are unitarily equivalent with $L_{2}(\Delta ; E F)$ and $L_{2}\left(\Delta ; E F^{-1}\right)$, respectively. In what follows we shall not distinguish between the abstractly defined $H_{1}$ and $H_{2}$ and their realizations $L_{2}(\Delta ; E F)$ and $L_{2}\left(\Delta ; E F^{-1}\right)$. Thus for $y^{1} \in L_{2}(\Delta ; E F)$ and $z^{2} \in L_{2}\left(\Delta ; E F^{-1}\right)$ we have

$$
\left\langle y^{1}, z^{2}\right\rangle=\int_{\Delta}\left(E y^{1}, z^{2}\right) d \chi
$$

and the bilinear form $Q$ is defined equivalently by (1.3.2) and (1.12).

Next we define the transformation $L_{21}^{00}$ as in (1.11). Then $\mathfrak{D}\left(L_{21}^{00}\right)$ is dense in $H_{1}$ and an integration by parts shows that $Q(y, z)=0$ for all $y, z \in(B)\left(L_{21}^{00}\right)$. Thus $B\left(L_{21}^{00}\right)$ is a negative subspace of $H_{12}$ with dense domain and hence, by Lemma 1.3.1, its closure, which we denote by $N_{0}$, is also a graph, say of the transformation $L_{21}^{0}$. It follows from the continuity of $Q$ that $N_{0}$ is a null space in the sense of Definition 1.5.1. In the present development, the operator $L_{0}$ engendered (see Definition 1.3.3) by $L_{21}^{0}$ will be called the minimal operator of the spatial part of the hyperbolic operator (1.2). Lemma 1.5.6 shows that 
$L_{0}$ is closed. Further, it is clear that $L_{0}$ is an extension of

$$
\begin{aligned}
L_{00} y^{0} & =E^{-1}\left[\left(A^{i} y^{0}\right)_{i}+B y^{0}\right], \\
\mathcal{D}\left(L_{00}\right) & =\left[y^{0} ; y^{0} \text { continuously differentiable with compact carrier in } \Delta\right],
\end{aligned}
$$

as can be seen from the fact that $L_{00}=I_{20}^{-1} L_{21}^{00} I_{01}^{-1}$. This latter relation also shows that $\mathfrak{D}\left(I_{20} L_{0} I_{01}\right) \supset \mathfrak{D}\left(L_{21}^{00}\right)$ and hence that $\mathfrak{D}\left(I_{21} L_{0} I_{01}\right)$ is dense in $H_{1}$. Incidently, we conjecture that $L_{0}$ is not the smallest closed extension of $L_{00}\left({ }^{7}\right)$. However, from our point of view this is all to the good since we want $L_{0}$ to be the largest operator on $H_{0}$ (and $L_{21}^{0}$ to be the largest transformation on $H_{1}$ to $H_{2}$ ) of the type $E^{-1}\left[\left(A^{i} y^{j}\right)_{i}+B y^{j}\right], j=0$ (and 1 ), for which the domain functions have "essentially" zero boundary data. Finally we note that $\mathfrak{D}\left(L_{00}\right)$ is dense in $H_{0}$ and consequently so is $\mathfrak{D}\left(L_{0}\right)$.

We also define the formal adjoints of these operators in an entirely analogous manner and it is clear that corresponding operators will possess the same properties. For instance, we define

$$
M_{21}^{00} z^{1}=E^{-1}\left[-\left(A^{i} z^{1}\right)_{i}+\left(B^{*}+A_{i}^{i}\right) z^{1}\right]
$$

with $\mathfrak{D}\left(M_{21}^{00}\right)=\mathfrak{D}\left(L_{21}^{00}\right)$. It is readily verified that $\mathfrak{S}\left(M_{21}^{00}\right)=U\left[\mathfrak{S}\left(L_{21}^{00}\right)\right]$ and, since $U$ is bicontinuous, this relation continues to hold for the closures of these graphs. Thus if $M_{21}^{0}$ denotes the smallest closed linear extension of $M_{21}^{00}$, then $\left(\mathfrak{S}\left(M_{21}^{0}\right)=U\left[\$\left(L_{21}^{0}\right)\right]\right.$ and it follows that $(\mathfrak{S})\left(M_{21}^{0}\right)$ is also a null space along with $\mathfrak{S}\left(L_{21}^{0}\right)$. It is also evident from the definition of $U$ that $\mathfrak{D}\left(M_{21}^{0}\right)=\mathfrak{D}\left(L_{21}^{0}\right)$. However, the domain of the operator $M_{0}$ engendered by $M_{21}^{0}$ will in general not coincide with the domain of $L_{0}$, engendered by $L_{21}^{0}$. We note that $\mathfrak{D}\left(M_{0}\right)$ is dense in $H_{0}$ and that $\mathfrak{D}\left(I_{20} M_{0} I_{01}\right)$ is dense in $H_{1}$.

We now define $N_{1}$ to be the $Q$-orthogonal complement of $N_{0}$. Since $\mathfrak{D}\left(N_{0}\right)$ is dense in $H_{1}$, it follows from Lemma 1.4.3 that $N_{1}$ is also a graph, say of the transformation $L_{21}^{1}$. Further, $N_{0}$ being a null space, we conclude that $N_{1} \supset N_{0}$ and hence, that $L_{21}^{1} \supset L_{21}^{0}$. For $y=\left[y^{1}, y^{2}\right]$ and $z=\left[z^{1}, z^{2}\right]$ we see that $y \in N_{1}$ if and only if

$$
\begin{aligned}
0=Q(y, z) & =\left\langle y^{1}, L_{21}^{0} z^{1}\right\rangle+\left\langle y^{2}, z^{1}\right\rangle-\left\langle D_{21} y^{1}, z^{1}\right\rangle \\
& =-\left\langle y^{1}, M_{21}^{0} z^{1}\right\rangle+\left\langle y^{2}, z^{1}\right\rangle, \quad \\
& z^{1} \in \mathfrak{D}\left(M_{21}^{0}\right) .
\end{aligned}
$$

Thus $L_{21}^{1}$ can be thought of as the "adjoint" of $M_{21}^{0}$ relative to this mixed inner product; in the usual notation for adjoint we have $L_{21}^{1}=F_{21}\left(M_{21}^{0}\right) * F_{21}$

$\left({ }^{7}\right)$ Assuming the above conjecture to be correct, our use of the term "minimal operator" differs from the customary usage (cf. L. Hörmander [8]), which applies the term to the smallest closure of $L_{00}$, say $L_{0}^{\prime}$. On the other hand, $L_{0}^{\prime}$ would be a more appropriate minimal operator than our $L_{0}$ if we wished to determine all maximal dissipative extensions of a minimal operator; likewise, $L_{1}^{\prime}=\left(M_{0}^{\prime}\right)^{*}$ would be a more appropriate maximal operator than our $L_{1}$ if we wished to determine all maximal dissipative restrictions of a maximal operator. 
since $M_{21}^{0}$ is the smallest closed extension of $M_{21}^{00}$, it is clear that $y$ will belong to $\mathfrak{S}\left(L_{21}^{1}\right)$ if and only if (2.1.5) merely holds for all $z^{1} \in \mathfrak{D}\left(M_{21}^{00}\right)$. Now if $y^{1} \in L_{2}(\Delta ; E F)$ is continuously differentiable on $\Delta$ and if

$$
y^{2}=E^{-1}\left[\left(A^{i} y^{1}\right)_{i}+B y^{1}\right] \in L_{2}\left(\Delta ; E F^{-1}\right),
$$

then one sees by direct computation that (2.1.5) will be satisfied for all $z^{1} \in \mathfrak{D}\left(M_{21}^{00}\right)$; thus $y=\left[y^{1}, y^{2}\right]$ belongs to $\mathcal{B}\left(L_{21}^{1}\right)$ and so does the closure of this set of function pairs. A complete description of $L_{21}^{1}$ is given in the following.

Lemma 2.1.1. Suppose that the elements of $E$, the $A^{i}$, and $B$ are continuously

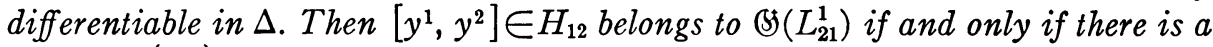
sequence $\left\{y_{n}^{1}\right\} \subset \mathfrak{D}\left(L_{21}^{00}\right)$ such that

$$
\left[y_{n}^{1}, L_{21}^{00} y_{n}^{1}\right] \rightarrow\left[y^{1}, y^{2}\right] \text { in } L_{2}(\Lambda ; E F) \times L_{2}\left(\Lambda ; E F^{-1}\right)
$$

for each compact $\Lambda \subset \Delta$.

Proof. The sufficiency argument is trivial in view of the fact that (2.1.5) need only be verified for $z^{1} \in \mathfrak{D}\left(M_{21}^{00}\right)$ and each such $z^{1}$ has a compact support in $\Delta$. The necessity can be established by a method developed by $\mathrm{K}$. O. Friedrichs [6, pp. 365-373]. The reduction of the present problem to that treated by Friedrichs is readily accomplished by means of the following unitary transformations:

$$
\begin{aligned}
& S: \quad y^{1} \rightarrow y=(E F)^{1 / 2} y^{1}, \quad L_{2}(\Delta ; E F) \quad \text { onto } L_{2}(\Delta ; I), \\
& T: \quad z^{2} \rightarrow z=(E F)^{-1 / 2} E z^{2}, \quad L_{2}\left(\Delta: E F^{-1}\right) \text { onto } L_{2}(\Delta ; I) \text {. }
\end{aligned}
$$

These are multiplicative transformations and since the factors are continuously differentiable, it is clear that continuously differentiable functions map onto functions of the same kind and likewise functions with compact support map onto functions having the same support. Setting

$$
\begin{aligned}
\mathfrak{L}^{1} & =T L_{21}^{1} S^{-1}, \\
\mathcal{L}^{00} y & =T L_{21}^{00} S^{-1} y=\left(Q^{i} y\right)_{i}+B y, \\
\mathfrak{T}^{00} z & =T M_{21}^{00} S^{-1} z=-\left(Q^{i} z\right)_{i}+\left(B^{*}+Q_{i}^{i}\right) z,
\end{aligned}
$$

where

$$
\begin{aligned}
Q^{i} & =(E F)^{-1 / 2} A^{i}(E F)^{-1 / 2} \text { and } \\
B & =(E F)^{-1 / 2} B(E F)^{-1 / 2}-\left[(E F)^{-1 / 2}\right]_{i} A^{i}(E F)^{-1 / 2},
\end{aligned}
$$

it is clear that

$\mathfrak{D}\left(\mathfrak{N}^{00}\right)=\mathfrak{D}\left(\mathfrak{L}^{00}\right)=[y ; y$ continuously differentiable with compact support in $\Delta]$.

Moreover, $[y, f] \in\left(S\left(\mathcal{L}^{1}\right)\right.$ if and only if 


$$
\int_{\Delta}\left(y, \mathfrak{N}^{00} z\right) d \chi=\int_{\Delta}(f, z) d \chi \quad \text { for all } z \in \mathfrak{I}\left(\mathfrak{T}^{00}\right) .
$$

The main steps in Friedrichs' argument are as follows. He begins with a function $j\left(\chi^{1}\right)$ possessing derivatives of all orders and such that $j\left(\chi^{1}\right) \geqq 0$, $j\left(\chi^{1}\right)=0$ for $\left|\chi^{1}\right| \geqq 1$, and $\int_{-1}^{1} j\left(\chi^{1}\right) d \chi^{1}=1$. Setting

$$
j_{\epsilon}(\chi)=\epsilon^{-m} \prod_{i=1}^{m} j\left(\epsilon^{-1} \chi^{i}\right),
$$

he defines the mollifier operator

$$
\left[\partial_{\epsilon} y\right](\chi)=\int_{\Delta} j_{\epsilon}(\chi-\bar{\chi}) y(\bar{\chi}) d \bar{\chi} .
$$

It is easy to show that both $\mathfrak{g}_{\epsilon}$ and $g_{\epsilon}^{*}$ approximate the identity in $L_{2}(\Delta ; I)$ as $\epsilon \rightarrow 0+$. For $z \in \mathfrak{D}\left(\mathfrak{T}^{00}\right)$ and $\epsilon$ sufficiently small, $\partial_{\epsilon}^{*} z \in \mathfrak{I}\left(\mathfrak{T}^{00}\right)$ so that (2.1.6) becomes

$$
\left\langle y, \mathfrak{M}^{00} \mathcal{g}_{\epsilon}^{*} z\right\rangle=\left\langle f, g_{\epsilon}^{*} z\right\rangle=\left\langle\mathcal{J}_{f} f, z\right\rangle .
$$

Now $\mathfrak{M}^{00} g_{\epsilon}^{*}$ and $\mathscr{L}^{00} g_{\epsilon}$ are integral operators with kernels $k_{\epsilon}^{1}(\chi, \bar{\chi})$ and $k_{\epsilon}^{2}(\chi, \bar{\chi})$, respectively. Setting

$$
\left[\mathcal{K}_{\epsilon} y\right](\chi)=\int_{\Delta}\left[k_{\epsilon}^{1}(\bar{\chi}, \chi)^{*}-k_{\epsilon}^{2}(\chi, \bar{\chi})\right] y(\bar{\chi}) d \bar{\chi}
$$

and considering $\mathcal{K}_{\epsilon}$ as a transformation on $L_{2}(\Delta ; I)$ to $L_{2}(\Lambda ; I)$, Friedrichs shows that $\mathcal{K}_{\epsilon} y \rightarrow \theta$ as $\epsilon \rightarrow 0$ for each compact $\Lambda \subset \Delta$. To complete the proof, we choose a sequence of domains $\left\{\Delta_{n}\right\}$ such that $\bar{\Delta}_{n} \subset \Delta_{n+1} \subset \Delta$ and $\Delta=\bigcup_{n} \Delta_{n}$. Suppose that $\Delta_{n}$ is a distance greater than $\delta_{n}>0$ from the complement of $\Delta$ and suppose further that $\delta_{n} \rightarrow 0$. For $\epsilon_{n}=\delta_{n} / 4$, we define

$$
\begin{array}{rlrl}
y_{n}(\chi) & =\left[\mathfrak{d}_{\epsilon_{n}} y\right](\chi), & & \chi \in \Delta_{n}, \\
& =0, & \chi \in \Delta_{n+1},
\end{array}
$$

and elsewhere $y_{n}(\chi)$ is defined so as to be continuously differentiable in $\Delta$. Then $\left\{y_{n}\right\} \subset \mathfrak{I}\left(\mathfrak{L}^{00}\right)$ and $y_{n} \rightarrow y$ in $L_{2}(\Lambda ; I)$ for each compact $\Lambda \subset \Delta$. Moreover, according to (2.1.7)

$$
\left\langle\left(\mathcal{S}_{\epsilon_{n}} f-\mathfrak{L}^{00} y_{n}\right), z\right\rangle=\left\langle\mathcal{K}_{\epsilon_{n}} y, z\right\rangle
$$

for each $z \in \mathfrak{I}\left(\mathfrak{T}^{00}\right)$ with support contained in $\Delta_{n}$. It follows that $\mathfrak{L}^{00} y_{n}$ $=\mathcal{J}_{\epsilon_{n}} f-\mathcal{K}_{\epsilon_{n}} y$ for all $\chi \in \Delta_{n}$ and hence that $\mathscr{L}^{00} y_{n} \rightarrow f$ in $L_{2}(\Lambda ; I)$, again for each compact $\Lambda \subset \Delta$. It readily follows that the sequence $\left\{S^{-1} y_{n}\right\} \subset \mathfrak{D}\left(L_{21}^{00}\right)$ satisfies the assertion of the lemma.

Returning to the main thread of our argument, $M_{21}^{1}$ is defined in a manner analogous to $L_{21}^{1}$ as the transformation whose graph is the $Q$-orthogonal 
complement of $B\left(M_{21}^{0}\right)$. It is clear from the properties of $U$ that $B\left(M_{21}^{1}\right)$ $=U\left[S\left(L_{21}^{1}\right)\right]$ and it follows from this that the analogue of Lemma 2.1.1 holds for $M_{21}^{1}$. Finally let $L_{1}$ and $M_{1}$ be the operators engendered by $L_{21}^{1}$ and $M_{21}^{1}$, respectively. According to Lemma 1.5.7, $L_{1} \subset M_{0}^{*}$ and $M_{1} \subset L_{0}^{*}$ and we suspect that in general these inclusions are proper. We shall call $L_{1}$ the maximal operator of the spatial part of the hyperbolic operator (1.2). It follows from the way in which $L_{1}$ was defined, that among the differential operators of this type it possesses the largest domain to which the boundary integral (1.9) can be meaningfully extended. Consequently, any generator of properly dissipative solutions of (1.2) must necessarily be a restriction of $L_{1}$. An analogous assertion holds for $M_{1}$.

Next we introduce the boundary spaces for $L_{1}$ and $M_{1}$, namely the quotient spaces

$$
H=\leftrightarrow\left(L_{21}^{1}\right) / \mathscr{S}\left(L_{21}^{0}\right) \text { and } H^{\prime}=\leftrightarrow\left(M_{21}^{1}\right) / \mathscr{S}\left(M_{21}^{0}\right),
$$

respectively. These cosets correspond to sets of functions in $\mathfrak{D}\left(L_{21}^{1}\right)$ and $\mathfrak{D}\left(M_{21}^{1}\right)$, respectively, which exhibit the same boundary behavior. Now $\boldsymbol{H}$ can be represented by $\leftrightarrow\left(L_{21}^{1}\right) \cap\left(S\left(L_{21}^{0}\right)^{\perp}\right.$ and under this representation the homomorphism is simply the projection $\beta$ of $\leftrightarrow\left(L_{21}^{1}\right)$ onto $\mathscr{S}\left(L_{21}^{1}\right) \cap \Im\left(S\left(L_{21}^{0}\right)^{\perp}\right.$. Similarly $H^{\prime}$ has the representation $\circlearrowleft\left(M_{21}^{1}\right) \cap \circlearrowleft\left(M_{21}^{0}\right)^{\perp}$ and under this representation the homomorphism takes the form of the projection $\beta^{\prime}$ of $B\left(M_{21}^{1}\right)$ onto $\mathfrak{B S}\left(M_{21}^{1}\right) \cap \mathbb{B}\left(M_{21}^{0}\right)^{\perp}$.

As shown in $\$ 1.5$, the bilinear form $Q$ induces the bilinear forms $Q$ and $Q^{\prime}$ on $H$ and $H^{\prime}$, respectively. Finally we recall that the transformation $U$ (see $\$ 1.6$ ) on $H$ to $H^{\prime}$ is one-to-one, bicontinuous, and takes maximal negative (maximal positive) subspaces of $\boldsymbol{H}$ into maximal positive (maximal negative) subspaces of $H^{\prime}$ in a one-to-one fashion. With all this in mind, we now state our principal result.

THEOREM 2.1.1. There is a one-to-one correspondence between the maximal negative subspaces $[\boldsymbol{N}]$ of $\boldsymbol{H}$, taken with respect to $\boldsymbol{Q}$, and the closed maximal dissipative operators $[L]$ on $H_{0}$ such that $L_{0} \subset L \subset L_{1}$, the correspondence being defined by

$$
\mathfrak{D}(L)=\left[y^{0} ; y^{0} \in \mathfrak{D}\left(L_{1}\right) \text { and } \beta\left[I_{01}^{-1} y^{0}, I_{20} L_{1} y^{0}\right] \in N\right],
$$

which is dense in $H_{0}$. The adjoint operator $M=L^{*}$ is again maximal dissipative with dense domain, $M_{0} \subset M \subset M_{1}$, and

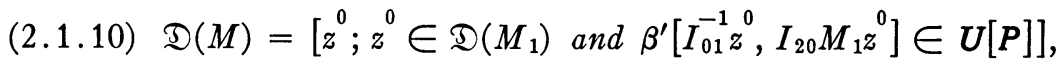

where $P$ is the $Q$-orthogonal complement of $N$ and $U[P]$ is a maximal negative subspace of $H^{\prime}$.

This theorem merely paraphrases Theorems 1.5 .1 and 1.6.1. It also char- 
acterizes all maximal and properly dissipative restrictions of $L_{1}$ as can be seen from the corollary to Theorem 1.5.1.

According to Lemma 1.5.8 we can obtain a conservative extension of $L_{0}$ only if $F=I$, that is, only if $D(\chi)=\Theta$ for all $\chi \in \Delta$. In this case $L_{0}$ is the smallest closed extension of $L_{00}$ and $L_{1}=-L_{0}^{*}$ Theorem 1.5.2 then gives a characterization of the conservative maximal dissipative extensions of $L_{0}$ as well as the self-adjoint extensions of $i L_{0}$.

2.2. Coupled systems. In order to treat dissipative hyperbolic systems with boundary conditions of the "elastic" type by the above method, it is necessary to take into account the interchange of energy between the main system and the boundary system. Because of this energy interchange the central system by itself is no longer dissipative. Nevertheless the coupled system is dissipative and hence one is lead to treat the entire coupled system as a unit. This is our starting point; the reader will find further motivation for the material in this section in $[12, \S 7]$.

Much work remains to be done before one can be sure that the problems treated in this chapter and especially those treated in this section have been correctly formulated. In view of this fact we have been content to study a rather simple boundary system.

The uncoupled components of our system will consist of the central system considered in the previous section, acting in the space $H_{0}$, and a boundary system acting in a hilbert space $\dot{H}_{0}$ and governed by the equation

$$
\dot{y}_{t}^{0}=B \dot{y}^{0},
$$

where $\dot{B}$ is a bounded linear operator.

To the right member of (2.2.1) we now add a coupling term of the form $C y^{i}$ where $C$ is a closed linear operator acting on the boundary value assumed at the time in the central system, namely

$$
y^{i}=\beta\left[I_{01}^{-1} y^{0}, I_{20} L_{1} y^{0}\right] \text {. }
$$

Thus the coupled system acts in the space $\mathfrak{S}_{0} \equiv H_{0} \times \dot{H}_{0}$ with elements $\mathfrak{y}^{0}$ $=\left[y^{0}, \dot{y}^{0}\right]$ and inner product

$$
\left\langle y^{0}, z^{0}\right\rangle=\left\langle y^{0}, z^{0}\right\rangle+\left\langle\dot{y}^{0}, \dot{z}^{0}\right\rangle
$$

and it is governed by the equation

$$
\mathfrak{y}_{t}^{0}=\mathfrak{R}_{1} \mathfrak{y}^{0}
$$

where

$$
\mathfrak{R}_{1} \mathfrak{y}^{0}=\left[L_{1} y^{0}, \dot{B} \dot{y}^{0}+C y^{i}\right]
$$

and, roughly speaking, $\mathfrak{D}\left(\Omega_{1}\right)$ consists of those elements $\mathfrak{y}^{0}$ for which $y^{0} \in \mathfrak{D}\left(L_{1}\right)$, $y^{i} \in \mathfrak{D}(C)$, and $\dot{y}^{0} \in \dot{H}_{0}$. A precise description of $\mathfrak{D}\left(\Omega_{1}\right)$ will appear in the 
course of the subsequent development.

We shall impose the following conditions on $C$ :

(i) There exists a subset $S$ of $\mathfrak{D}\left(C W C^{*}\right)$ with $\left[\left[\dot{y}^{0}, W C^{*} \dot{y}^{0}\right] ; \dot{y}^{0} \in S\right]$ dense in the graph of $W C^{*}$ such that $W C^{*}[S] \subset \beta\left[\$\left(I_{20} L_{1} I_{01}\right)\right]$;

(ii) $\dot{B}+\dot{B}^{*}-C W C^{*} \leqq \Theta$ on $\mathfrak{D}\left(C W C^{*}\right)$.

When $F=I$ Condition (i) is not very restrictive. For in this case $\beta\left[\$\left(I_{20} L_{1} I_{01}\right)\right]=H$ and the condition simply requires that the set $\left[\left[\dot{y}^{0}, W C^{*} \dot{y}^{0}\right]\right.$; $\left.\dot{y}^{0} \in \mathfrak{D}\left(C W C^{*}\right)\right]$ be dense in the graph of $W C^{*}$. However if $\mathfrak{D}\left(C W C^{*}\right)$ is merely dense in $\dot{H}_{0}$, then we can take a restriction $G_{0}$ of $W C^{*}$ with domain $\mathfrak{D}\left(C W C^{*}\right)$ and redefine $C$ as $G_{0}^{*} W^{-1}$. It is readily verified that the so-defined $C$ is a closed extension of the original $C$ and satisfied (i) in its entirety.

Condition (ii) is a dissipative condition similar to (1.3). In fact, let us imagine the central system as extending beyond the boundary of $\Delta$. Then at the boundary of $\Delta$ there will be a discontinuity in $y^{0}$ due to the back reaction of the coupling. Thus to a given $y^{0}$ there is associated an interior boundary value $\boldsymbol{y}^{i}$ defined by (2.2.2) and an exterior boundary value $\boldsymbol{y}^{\boldsymbol{e}}$ given by

$$
y^{e}=y^{i}+G \dot{y}^{0},
$$

where $G$ can be thought of as a back coupling operator describing the effect of the boundary system on the central system.

Next we assume that $C$ and $G$ are so related that no energy is lost in the coupling itself. In order to see what this means, let $\mathfrak{y}^{0}(t)$ be a solution of (2.2.3). Then the rate of increase of energy in the system is readily computed as

$$
\begin{aligned}
\left\langle\mathfrak{y}^{0}, \mathfrak{y}^{0}\right\rangle_{t}= & \int_{\Delta}\left(E D y^{0}, y^{0}\right) d \chi+Q\left(y^{i}, y^{i}\right)+\left\langle\left(\dot{B}+\dot{B}^{*}\right) \dot{y}^{0}, \dot{y}^{0}\right\rangle \\
& +\left\langle C y^{i}, \dot{y}^{0}\right\rangle+\left\langle\dot{y}^{0}, C y^{i}\right\rangle .
\end{aligned}
$$

Replacing $\boldsymbol{Q}\left(\boldsymbol{y}^{i}, \boldsymbol{y}^{i}\right)$ by its equivalent,

$$
\boldsymbol{Q}\left(\boldsymbol{y}^{e}, \boldsymbol{y}^{e}\right)-\boldsymbol{Q}\left(G \dot{y}^{0}, G \dot{y}^{0}\right)-\boldsymbol{Q}\left(\boldsymbol{y}^{i},\left(\xi \dot{y}^{0}\right)-\boldsymbol{Q}\left(G \dot{y}^{0}, \boldsymbol{y}^{i}\right),\right.
$$

we see that $\left\langle\mathfrak{y}^{0}, \mathfrak{y}^{0}\right\rangle_{t}$ is expressible in terms of the rate of increase of energy in the interior of the central and boundary systems plus the flow of energy through the exterior boundary provided that the cross product terms nullify each other. Thus our assumption amounts to setting

$$
\boldsymbol{Q}\left(\boldsymbol{y}^{i}, G \dot{y}^{0}\right)+\boldsymbol{Q}\left(G \dot{y}^{0}, y^{i}\right)=\left\langle C y^{i}, \dot{y}^{0}\right\rangle+\left\langle\dot{y}^{0}, C y^{i}\right\rangle
$$

for all $y^{i} \in \mathfrak{D}(C)$ and $\dot{y}^{0} \in \mathfrak{D}(G)$. We shall see below that this requires that $G=W C^{*}$ and hence the rate of increase of energy due to sources interior to the boundary system is

$$
\left\langle\left(B+B^{*}\right) \dot{y}^{0}, \dot{y}^{0}\right\rangle-Q\left(G \dot{y}^{0}, G \dot{y}^{0}\right)=\left\langle\left(B+\dot{B}^{*}-C W C^{*}\right) \dot{y}^{0}, \dot{y}^{0}\right\rangle .
$$

Finally if we assume that the boundary system is dissipative, we arrive at Condition (ii). 
Lema 2.2.1. The assumption (2.2.7) is equivalent with

$$
C=G^{*} W^{-1} \text { and } G=W C^{*} .
$$

Proof. Since (2.2.7) holds for both $\dot{y}^{0}, y^{i}$ and $i \dot{y}^{0}, y^{i}$, it is equivalent with $Q\left(y^{i}, G \dot{y}^{0}\right)=\left\langle C y^{i}, \dot{y}^{0}\right\rangle$ and hence by (1.6.4) with

$$
\left\langle W^{-1} y^{i}, G \dot{y}^{0}\right\rangle=\left\langle C y^{i}, \dot{y}^{0}\right\rangle
$$

for all $y^{i} \in \mathfrak{D}(C)$ and $\dot{y}^{0} \in \mathfrak{D}(G)$. However the relation (2.2.9) is valid if and only if $C=G^{*} W^{-1}$, or, what amounts to the same thing, if and only if $G=W C^{*}$.

After this digression, we return to the main line of argument. The operator

$$
D_{0}=\dot{B}+\dot{B}^{*}-C W C^{*}
$$

with domain $\mathfrak{D}\left(C W C^{*}\right)$ is clearly symmetric and by assumption negative. We can therefore apply the Friedrichs' extension theorem to obtain a selfadjoint extension $\dot{D} \leqq \Theta$ (see, for instance, [11, §VI.2]). Then, proceeding as in $\$ 1.2$, we construct the dual hilbert spaces $\dot{H}_{1}$ and $\dot{H}_{2}$ from the positive definite operator $\dot{F}=I-\dot{D}$ and define the transformations $\dot{F}_{21}, \dot{F}_{12}, \dot{D}_{21}, \dot{I}_{01}, \dot{I}_{20}$, and $\dot{I}_{21}$. It follows from the manner in which the Friedrichs' extension is obtained that $\mathfrak{D}\left(C W C^{*}\right) \subset \dot{I}_{01}\left[\dot{H}_{1}\right]$ and further that $\dot{I}_{01}^{-1}\left[\mathfrak{D}\left(C W C^{*}\right)\right]$ is dense in $\dot{H}_{1}$. Moreover $\mathfrak{D}(G) \subset \dot{I}_{01}\left[\dot{H}_{1}\right]$. In fact according to Condition (i), for each $\dot{y}^{0} \in \mathfrak{D}(G)$ there exists a sequence $\left\{\dot{y}_{n}^{0}\right\} \in \mathfrak{D}\left(C W C^{*}\right)$ such that $\left[\dot{y}_{n}^{0}, G \dot{y}_{n}^{0}\right]$ $\rightarrow\left[\dot{y}^{0}, G \dot{y}^{0}\right]$. Thus

$$
\begin{aligned}
\left\|\dot{y}_{n}^{0}-\dot{y}_{m}^{0}\right\|_{1}^{2}= & \left\langle\left(I-\dot{B}-\dot{B}^{*}\right)\left(\dot{y}_{n}^{0}-\dot{y}_{m}^{0}\right),\left(\dot{y}_{n}^{0}-\dot{y}_{m}^{0}\right)\right\rangle \\
& -\left\langle W^{-1} G\left(\dot{y}_{n}^{0}-\dot{y}_{m}^{0}\right), G\left(\dot{y}_{n}^{0}-\dot{y}_{m}^{0}\right)\right\rangle \rightarrow 0
\end{aligned}
$$

so that $\left\{\dot{y}_{n}^{0}\right\}$ defines an element of $\dot{H}_{1}$ whose $\dot{I}_{01}$ image is just $\dot{y}^{0}$.

Setting

$$
B_{21}=\dot{I}_{20} \dot{B} \dot{I}_{01} \quad \text { and } \quad \dot{B}_{21}^{*}=I_{20} \dot{B}^{*} \dot{I}_{01}
$$

it readily follows that $\dot{B}_{21}$ and $\dot{B}_{21}^{*}$ are bounded operators satisfying the relation

$$
\left\langle\dot{B}_{21} \dot{y}^{1}, \dot{z}^{1}\right\rangle=\left\langle\dot{y}^{1}, \dot{B}_{21}^{*} \dot{z}^{1}\right\rangle
$$$$
\dot{y}^{1}, \dot{z}^{1} \in \dot{H}_{1} .
$$

Further it is clear that $\dot{B}_{21}$ and $\dot{B}_{21}^{*}$ engender, respectively, restrictions of $\dot{B}$ and $\dot{B}^{*}$ with domain $\dot{I}_{01}\left[\dot{H}_{1}\right]$. We also note that the operator

$$
[C G]_{21} \equiv \dot{F}_{21}-I_{21}+\dot{B}_{21}+\dot{B}_{21}^{*}
$$

is bounded and symmetric on $\dot{H}_{1}$ to $\dot{H}_{2}$ and extends $\dot{I}_{20} C W C^{*} \dot{I}_{01}^{-1}$ whose domain, as we have already remarked, is dense in $\dot{H}_{1}$. Finally we set 


$$
D_{21}=B_{21}+\dot{B}_{21}^{*}-[C G]_{21}
$$

and define the quadratic form $\dot{Q}$ on $\dot{H}_{12}=\dot{H}_{1} \times \dot{H}_{2}$ as

$$
\dot{Q}(\dot{y}, \dot{z})=\left\langle\dot{y}^{1}, \dot{z}^{2}\right\rangle+\left\langle\dot{y}^{2}, \dot{z}^{1}\right\rangle-\left\langle\dot{D}_{21} \dot{y}^{1}, \dot{z}^{1}\right\rangle .
$$

We now define

$$
G_{1}=G \dot{I}_{01}
$$

Then $G$ is engendered by $G_{1}$ (since $\mathfrak{D}(G) \subset \dot{I}_{01}\left[\dot{H}_{1}\right]$ ), $\mathfrak{D}\left(G_{1}\right)$ is dense in $\dot{H}_{1}$ with $\dot{I}_{01}^{-1}\left[\mathfrak{D}\left(C W C^{*}\right)\right]$, and $G_{1}$ is closed with $G$. Next we set

$$
C_{1}=\dot{F}_{21} G_{1}^{*} W^{-1} \text {. }
$$

Then $C_{1}$ is also closed by virtue of the fact that $W^{-1}$ is bounded, $G_{1}^{*}$ is closed, and $\dot{F}_{21}$ is unitary. It is clear that $G_{1}^{*} \supset \dot{I}_{01}^{*} G^{*}$. On the other hand $\dot{F}_{21} \dot{I}_{01}^{*}=\dot{I}_{20}$ as can be seen from

$$
\left\langle\dot{I}_{20} \dot{y}^{0}, \dot{z}^{1}\right\rangle=\left\langle\dot{y}^{0}, \dot{I}_{01} \dot{z}^{1}\right\rangle=\left\langle\dot{I}_{01}^{*} \dot{y}^{0}, \dot{z}^{1}\right\rangle_{1}=\left\langle\dot{F}_{21} \dot{I}_{01}^{*} \dot{y}^{0}, \dot{z}^{1}\right\rangle ;
$$

and therefore $C_{1} \supset \dot{I}_{20} C$. Hereafter we shall denote the operator engendered by $C_{1}$ by the symbol $C_{0}$. It follows that $C_{0} \supset C$ and also that $C_{1} G_{1} \supset \dot{I}_{20} C G_{1}$ $=\dot{I}_{20} C G \dot{I}_{01}$. Finally we show that

$$
\mathfrak{I}\left(C_{1}\right) \supset \mathfrak{R}\left(G_{1}\right) .
$$

In fact, we conclude from Condition (i) that each element in the graph of $G_{1}$ can be approximated by a sequence $\left[\dot{y}_{n}^{1}, G_{1} \dot{y}_{n}^{1}\right]$ where $\left\{\dot{y}_{n}^{1}\right\} \subset \dot{I}_{01}^{-1}[\mathfrak{D}(C G)]$ and since $\dot{I}_{20} C G_{1}$ has the bounded extension $[C G]_{21}$, we see that $\lim C_{1} G_{1} \dot{y}_{n}^{1}$ also exists and hence that $\lim G_{1} \dot{y}_{n}^{1} \in \mathfrak{D}\left(C_{1}\right)$. It also follows that $C_{1} G_{1} \subset[C G]_{21}$.

With these preliminaries out of the way, we now proceed to apply the theory set forth in Chapter I. The operator $\mathfrak{F}$ on $\mathfrak{S}_{0}$ is defined as

$$
\mathfrak{F}=\left(\begin{array}{ll}
F & \Theta \\
\Theta & \dot{F}
\end{array}\right)
$$

where $\mathfrak{D}(\mathfrak{F})=\mathfrak{D}(F) \times \mathfrak{D}(\dot{F})$. The dual spaces $\mathfrak{S}_{1}$ and $\mathfrak{W}_{2}$ are constructed as in $\S 1.2$ and it is readily verified that $\mathfrak{S}_{1}=H_{1} \times \dot{H}_{1}$ and $\mathfrak{S}_{2}=H_{2} \times \dot{H}_{2}$. We denote the elements of $\mathfrak{S}_{1}$ and $\mathfrak{S}_{2}$ by $\mathfrak{y}^{1}=\left[y^{1}, \dot{y}^{1}\right]$ and $\mathfrak{y}^{2}=\left[y^{2}, \dot{y}^{2}\right]$, respectively. We also obtain the transformations $\mathfrak{F}_{21}, \mathfrak{F}_{12}, \mathfrak{D}_{21}, \mathfrak{\Im}_{01}, \mathfrak{\Im}_{20}, \mathfrak{\Im}_{21}$, which are all of the form

$$
\mathfrak{I}_{i j}=\left(\begin{array}{ll}
T_{i j} & \Theta \\
\Theta & \dot{T}_{i j}
\end{array}\right) \text {. }
$$

Finally we define the quadratic form on $\mathfrak{S}_{12}=\mathfrak{S}_{1} \times \mathfrak{S}_{2}$ as

$(2.2 .20) \quad \mathfrak{Q}(\mathfrak{y}, \mathfrak{z})=\left\langle\mathfrak{y}^{1}, \mathfrak{z}^{2}\right\rangle+\left\langle\mathfrak{y}^{2}, \mathfrak{z}^{1}\right\rangle-\left\langle\mathfrak{D}_{21} \mathfrak{y}^{1}, z^{1}\right\rangle=Q(y, z)+Q(\dot{y}, \dot{z})$

and the operator 


$$
\mathfrak{U}\left[\mathfrak{y}^{1}, \mathfrak{y}^{2}\right]=\left[\mathfrak{y}^{1},-\mathfrak{y}^{2}+\mathfrak{D}_{21} \mathfrak{y}^{1}\right] .
$$

Next we define the transformation

$$
\mathfrak{R}_{21}^{0} \mathfrak{y}^{1}=\left[L_{21}^{1} y^{1}, B_{21} \dot{y}^{1}+C_{1} y^{i}\right]
$$

where $y^{i}=\beta\left[y^{1}, L_{21}^{1} y^{1}\right]$ and

$$
\mathfrak{D}\left(R_{21}^{0}\right)=\left[\left[y^{1}, \dot{y}^{1}\right] ; y^{1} \in \mathfrak{D}\left(L_{21}^{1}\right), \dot{y}^{1} \in \mathfrak{D}\left(G_{1}\right), y^{i}=-G_{1} \dot{y}^{1}\right] .
$$

We set $\mathfrak{R}_{0}=\mathfrak{S}\left(\mathfrak{R}_{21}^{0}\right)$ and let $\mathfrak{\Omega}_{0}$ denote the transformation engendered by $\mathfrak{R}_{21}^{0}$. It follows directly that

$$
\mathfrak{R}_{0} \mathfrak{y}^{0}=\left[L_{1} y^{0}, B \dot{y}^{0}+C_{0} y^{i}\right]
$$

where $\boldsymbol{y}^{i}$ is defined as in (2.2.2) and

$$
\mathfrak{D}\left(\Omega_{0}\right)=\left[\left[y^{0}, \dot{y}^{0}\right] ; y^{0} \in \mathfrak{D}\left(L_{1}\right), \dot{y}^{0} \in \mathfrak{D}(G), y^{i}=-\mathfrak{G} \dot{y}^{0} \in \mathfrak{D}\left(C_{0}\right)\right] .
$$

Lemma 2.2.2. $\mathfrak{N}_{0}$ is a closed null space.

Proof. Suppose $\left\{\mathfrak{y}_{n}^{1}\right\} \subset \mathfrak{D}\left(\mathfrak{l}_{21}^{0}\right), \quad \mathfrak{y}_{n}^{1} \rightarrow \mathfrak{y}^{1}, \quad$ and $\quad \mathfrak{R}_{21}^{0} \mathfrak{y}_{n}^{1} \rightarrow \mathfrak{g}^{2}=\left[g^{2}, \dot{g}^{2}\right]$. Then $y_{n}^{1} \rightarrow y^{1}$ and $L_{21}^{1} y_{n}^{1} \rightarrow g^{2}$, and since $L_{21}^{1}$ is a closed transformation it follows that $y^{1} \in \mathcal{D}\left(L_{21}^{1}\right)$, and $L_{21}^{1} y^{1}=g^{2}$, and $y_{n}^{i} \rightarrow y^{i}$. Thus $\dot{y}_{n}^{1} \rightarrow \dot{y}^{1}$ and $G_{1} \dot{y}_{n}^{1}=-y_{n}^{i} \rightarrow-y^{i}$, and since $G_{1}$ is closed $\dot{y}^{1} \in \mathfrak{D}\left(G_{1}\right)$ and $G_{1} y^{1}=-y^{i}$. Finally $C_{1} y_{n}^{i}=\left[\ell_{21}^{0} y_{n}^{1}\right]^{2}-\dot{B}_{21} \dot{y}_{n}^{1}$ also converges and since $C_{1}$ is closed the limit is $C_{1} y^{i}$. Consequently $\mathfrak{y}^{1} \in \mathfrak{D}\left(\mathfrak{R}_{21}^{0}\right)$ and $\mathbb{R}_{21}^{0} \mathfrak{y}^{1}=\mathfrak{g}^{2}$. This proves that $\mathfrak{R}_{0}$ is closed.

In order to show that $\mathfrak{N}_{0}$ is a null space, let $\mathfrak{y}, z \in \mathfrak{N}_{0}$. Then

$$
\mathfrak{Q}(\mathfrak{y}, z)=Q(y, z)+\left\langle\dot{y}^{1}, C_{1} z^{i}\right\rangle+\left\langle C_{1} y^{i}, \dot{z}^{1}\right\rangle+\left\langle\dot{y}^{1},[C G]_{21} \dot{z}^{1}\right\rangle .
$$

Now

$$
Q(y, z)=Q\left(y^{i}, z^{i}\right)=\left\langle G_{1} \dot{y}^{1}, W^{-1} G_{1} z^{1}\right\rangle=\left\langle\dot{y}^{1}, C_{1} G_{1} \dot{z}^{1}\right\rangle=\left\langle\dot{y}^{1},[C G]_{21} \dot{z}^{1}\right\rangle .
$$

Further

$$
\begin{aligned}
& \left\langle\dot{y}^{1}, C_{1} z^{i}\right\rangle=-\left\langle\dot{y}^{1}, C_{1} G_{1} \dot{z}^{1}\right\rangle=-\left\langle\dot{y}^{1},[C G]_{21} \dot{z}^{1}\right\rangle, \\
& \left\langle C_{1} y^{i}, \dot{z}^{1}\right\rangle=-\left\langle C_{1} G_{1} \dot{y}^{1}, \dot{z}^{1}\right\rangle=-\left\langle[C G]_{21} \dot{y}^{1}, \dot{z}^{1}\right\rangle=-\left\langle\dot{y}^{1},[C G]_{21} \dot{z}^{1}\right\rangle .
\end{aligned}
$$

Combining these relations with (2.2.26) we see that $\mathfrak{Q}(\mathfrak{y}, z)=0$, which was to be proved.

LEMma 2.2.3. $\mathfrak{D}\left(\mathfrak{\Im}_{20} \mathbb{R}_{0} \mathfrak{\Im}_{01}\right)$ and $\mathfrak{D}\left(\mathfrak{R}_{0}\right)$ are dense in $\mathfrak{S}_{1}$.

Proof. Since $\mathfrak{D}\left(\Im_{20} \Omega_{0} \Im_{01}\right) \subset \mathfrak{D}\left(\mathfrak{R}_{0}\right)$, it suffices to show that $\mathfrak{D}\left(\Im_{20} \mathfrak{R}_{0} \Im_{01}\right)$ is dense in $\mathfrak{S}_{1}$. To this end let $\mathfrak{g}^{1}=\left[\mathrm{g}^{1}, \dot{g}^{1}\right]$ be an arbitrary element of $\mathfrak{S}_{1}$ and let $\epsilon>0$ be given. As we have already noted, there is a $\dot{u}^{1} \in \mathfrak{D}\left(C G_{1}\right)$ such that $\left\|\dot{g}^{1}-\dot{u}^{1}\right\|_{1}<\epsilon$. According to Condition (i) there will exist a $\dot{y}^{1} \in \mathfrak{D}\left(C G_{1}\right)$ with $G_{1} \dot{y}^{1} \in \beta\left[\left(\mathcal{S}^{2}\left(I_{20} L_{1} I_{01}\right)\right] \cap \mathfrak{D}(C)\right.$ such that $\left\|\dot{I}_{01} \dot{u}^{1}-\dot{I}_{01} \dot{y}^{1}\right\|<\epsilon$ and $\left\|G_{1} \dot{u}^{1}-G_{1} \dot{y}^{1}\right\|<\epsilon$. It therefore follows as in (2.2.10) that $\left\|\dot{y}^{1}-\dot{u}^{1}\right\|_{1}^{2} \leqq(1+2\|\dot{B}\|) \epsilon^{2}+\left\|W^{-1}\right\| \epsilon^{2}$. For 
such a $\dot{y}^{1}$ there is a $v^{1} \in \mathfrak{D}\left(I_{20} L_{1} I_{01}\right)$ such that $v^{i}=-G_{1} \dot{y}^{1}$. We now approximate $\left(g^{1}-v^{1}\right)$ in $H_{1}$ by an element of $\mathfrak{D}\left(L_{21}^{0}\right)$, say $w^{1}$. Thus $w^{i}=\theta$ and hence on setting $y^{1}=v^{1}+w^{1}$, we see that $y^{i}=-G_{1} \dot{y}^{1} \in \mathfrak{D}\left(C_{0}\right)$ and that $y^{1}$ approximates $g^{1}$. It follows that $\mathfrak{y}^{1}=\left[y^{1}, \dot{y}^{1}\right] \in \mathfrak{D}\left(\Im_{20} \mathfrak{\Omega}_{0} \Im_{01}\right)$ and approximates $\mathfrak{g}^{1}$.

We now define $\mathfrak{R}_{1}$ to be the $\mathfrak{Q}$-orthogonal complement of $\mathfrak{R}_{0}$. As before we see by Lemma 1.4 .3 that $\mathfrak{N}_{1}$ is a graph, say of the transformation $\mathfrak{R}_{21}^{1}$. Obviously $\mathfrak{R}_{21}^{1} \supset \mathfrak{R}_{21}^{0}$ and somewhat less obviously we have

LEMMA 2.2.4. The transformation $\mathfrak{R}_{21}^{1}$ can be represented as

$$
\mathfrak{R}_{21}^{1} \mathfrak{y}^{1}=\left[L_{21}^{1} y^{1}, \dot{B}_{21} \dot{y}^{1}+C_{1} y^{i}\right]
$$

where $y^{i}=\beta\left[y^{1}, L_{21}^{1} y^{1}\right]$ and

$$
\mathfrak{D}\left(L_{21}^{1}\right)=\left[\left[y^{1}, \dot{y}^{1}\right] ; y^{1} \in \mathfrak{D}\left(L_{21}^{1}\right), y^{i} \in \mathfrak{D}\left(C_{1}\right), \dot{y}^{1} \in \dot{H}_{1}\right] .
$$

Proof. Suppose $y \in \mathfrak{N}_{1}$. Then for each $z \in \mathfrak{N}_{0}$

$$
0=\mathfrak{Q}(\mathfrak{y}, z)=Q(y, z)+Q(\dot{y}, \dot{z}) .
$$

It is clear that $N_{0} \times \theta \subset \mathfrak{M}_{0}$ and hence that $Q(y, z)=0$ for each $z \in N_{0}$. Consequently $y \in N_{1}=\oiint\left(L_{21}^{1}\right), y^{i}$ exists, and $Q(y, z)=\boldsymbol{Q}\left(\boldsymbol{y}^{i}, \boldsymbol{z}^{i}\right)=-\boldsymbol{Q}\left(\boldsymbol{y}^{i}, G_{1} \dot{z}^{1}\right)$. On the other hand

$$
Q(\dot{y}, \dot{z})=\left\langle\dot{y}^{2}, \dot{z}^{1}\right\rangle+\left\langle\dot{y}^{1}, B_{21} \dot{z}^{1}-C_{1} G_{1} \dot{z}^{1}\right\rangle-\left\langle\left(\dot{B}_{21}+\dot{B}_{21}^{*}-[C G]_{12}\right) \dot{y}^{1}, \dot{z}^{1}\right\rangle
$$

and since

$$
\left\langle\dot{y}^{1}, C_{1} G_{1} \dot{z}^{1}\right\rangle=\left\langle\dot{y}^{1},[C G]_{12} \dot{z}^{1}\right\rangle=\left\langle[C G]_{12} \dot{y}^{1}, \dot{z}^{1}\right\rangle
$$

we see that

$$
0=-\left\langle W^{-1} y^{i}, G_{1} \dot{z}^{1}\right\rangle+\left\langle\dot{y}^{2}-\dot{B}_{21} \dot{y}^{1}, \dot{z}^{1}\right\rangle
$$

for all $\dot{z}^{1} \in \mathfrak{D}\left(G_{1}\right)$. It follows that $W^{-1} y^{i} \in \mathfrak{D}\left(G_{1}^{*}\right)$, that is $y^{i} \in \mathfrak{D}\left(C_{1}\right)$ and $\left\langle W^{-1} y,{ }^{i} G_{1} \dot{z}^{1}\right\rangle=\left\langle C_{1} y^{i}, \dot{z}^{1}\right\rangle$. Making use of the fact that $\mathfrak{D}\left(G_{1}\right)$ is dense in $\dot{H}_{1}$, we conclude that $\dot{y}^{2}=\dot{B}_{21} \dot{y}^{1}+C_{1} y^{i}$. Thus each $\mathfrak{y} \in \mathfrak{R}_{1}$ lies in the graph of $\mathfrak{R}_{21}^{1}$ as given by (2.2.27) and (2.2.28); the converse assertion is readily verified by retracing the above steps.

As before we let $\Omega_{1}$ denote the operator engendered by $\mathfrak{R}_{21}^{1}$. It is readily seen that

$$
\mathfrak{R}_{1} y^{0}=\left[L_{1} y^{0}, B \dot{y}^{0}+C_{0} y^{i}\right]
$$

where

(2.2.30) $\mathfrak{D}\left(\mathfrak{R}_{1}\right)=\left[\left[y^{0}, \dot{y}^{0}\right] ; y^{0} \in \mathfrak{D}\left(L_{1}\right), y^{i} \in \mathfrak{D}\left(C_{0}\right), \dot{y}^{0} \in \dot{I}_{01}\left[\dot{H}_{1}\right]\right]$.

Having determined $\mathfrak{N}_{0}$ and $\mathfrak{N}_{1}$ we next study the boundary space $\mathfrak{g}$ $=\mathfrak{N}_{1} / \mathfrak{N}_{0}$ defined as in $\$ 1.5$ with generic element $\mathfrak{y}$. We denote the natural 
mapping $\mathfrak{y} \in \mathfrak{N}_{1} \rightarrow \mathfrak{y} \in \mathfrak{S}$ by $\boldsymbol{\beta}$ and again we see that the quadratic form $\mathfrak{Q}$ induces a quadratic form on $\mathfrak{\mathfrak { g }}$ with the property $\mathfrak{Q}(\mathfrak{y}, \mathfrak{z})=\mathfrak{Q}\left(\boldsymbol{\beta}_{\mathfrak{y}}, \boldsymbol{\beta}_{\mathfrak{z}}\right)$.

The boundary spaces $\mathfrak{F}$ and $H$ are not unrelated and under certain conditions they are equivalent. In order to study this relationship we first set (2.2.31) $\mathfrak{N}_{0}=\left[\mathfrak{y} ; \mathfrak{y} \in \mathfrak{N}_{1}, y^{i} \in \mathfrak{D}\left(C_{1}\right), \dot{y}^{1} \in \mathfrak{I}\left(G_{1}\right)\right]$ and $\mathfrak{H}_{o}=\mathfrak{\beta}\left[\mathfrak{N}_{o}\right]$.

Since $\mathfrak{N}_{o}$ consists only of cosets of $\mathfrak{R}_{o}$ we see that $\mathfrak{R}_{o}=\beta^{-1}\left[\mathfrak{H}_{o}\right]$. Further it is easy to show that $\mathfrak{R}_{o}$ is dense in $\mathfrak{R}_{1}$ and it follows from this that $\mathfrak{Y}_{o}$ is dense in $\mathfrak{H}$.

Lemma 2.2.5. The mapping $\beta y \rightarrow y^{e} \equiv y^{i}+G_{1} \dot{y}^{1}$ is a one-to-one linear map of $\mathfrak{S}$ o onto $\mathfrak{D}\left(C_{1}\right) \subset H$, under which

$$
\|\beta y\|^{2} \leqq\left\|y^{e}\right\|^{2}+\left\|C_{1} y^{e}\right\|^{2}
$$

and

$$
\mathfrak{L}_{(}\left(3 \mathfrak{y}, \beta_{z}\right)=Q\left(y^{e}, y^{e}\right) .
$$

Proof. The first assertion follows directly from the fact that $\mathfrak{y \rightarrow y ^ { e }}$ is a linear map on $\mathfrak{N}_{o}$ with kernel $\mathfrak{N}_{o}$ and, by (2.2.18), with image set $\mathfrak{D}\left(C_{1}\right)$. For a particular $\boldsymbol{y}^{e} \in \mathfrak{D}\left(C_{1}\right)$ choose $\mathfrak{y} \in \mathfrak{R}_{o}$ such that $\boldsymbol{y}^{i}=\boldsymbol{y}^{e}$ and $\dot{y}^{1}=\theta$. Then

$$
\|\beta \mathfrak{y}\|^{2} \leqq\|\mathfrak{y}\|^{2}=\|y\|^{2}+\left\|C_{1} y^{i}\right\|^{2} .
$$

Minimizing this expression over all $y \in N_{1}$ with $y^{i}=y^{e}$ we obtain the inequality (2.2.32). Finally for $\mathfrak{y}, z \in \mathfrak{N}_{o}$ we have

$$
\mathfrak{Q}(\mathfrak{y}, \mathfrak{z})=Q\left(y^{i}, z^{i}\right)+\left\langle C_{1} y^{i}, \dot{z}^{1}\right\rangle+\left\langle\dot{y}^{1}, C_{1} z^{i}\right\rangle+\left\langle[C G]_{12} \dot{y}^{1}, \dot{z}^{1}\right\rangle
$$

and in view of the fact that

$$
\begin{aligned}
\left\langle C_{1} y^{i}, \dot{z}^{1}\right\rangle & =\left\langle W^{-1} y^{i}, G_{1} \dot{z}^{1}\right\rangle=Q\left(y^{i}, G_{1} \dot{z}^{1}\right), \\
\left\langle[C G]_{12} \dot{y}^{1}, \dot{z}^{1}\right\rangle & =\left\langle C_{1} G_{1} \dot{y}^{1}, \dot{z}^{1}\right\rangle=\left\langle W^{-1} G_{1} \dot{y}^{1}, G_{1} \dot{z}^{1}\right\rangle=Q\left(G_{1} \dot{y}^{1}, G_{1} \dot{z}^{1}\right),
\end{aligned}
$$

we see that $\mathfrak{Q}\left(\mathfrak{g} \mathfrak{y}, \mathfrak{\beta}_{\mathfrak{z}}\right)=\mathfrak{Q}(\mathfrak{y}, \mathfrak{z})=\boldsymbol{Q}\left(\boldsymbol{y}^{e}, z^{e}\right)$.

Corollary. If $C$ is bounded, then $\mathfrak{N}_{o}=\mathfrak{N}_{1}$ and $\boldsymbol{\beta} \mathfrak{y} \rightarrow \boldsymbol{y}^{e}$ is a bicontinuous isomorphism of $\mathfrak{\mathfrak { K }}$ onto $\boldsymbol{H}$ under which (2.2.33) is valid.

Proof. If $C$ is bounded, then $\dot{F}$ is bounded and $\dot{H}_{1}$ and $\dot{H}_{2}$ are equivalent with $\dot{H}_{0}$ under the respective mappings $\dot{I}_{01}$ and $\dot{I}_{20}$. As a consequence, $C_{1}$ and $G_{1}$ are bounded with $\mathfrak{D}\left(C_{1}\right)=H$ and $\mathfrak{D}\left(G_{1}\right)=\dot{H}_{1}$. The above lemma therefore implies that $\beta \eta \rightarrow y^{e}$ is one-to-one and linear on all of $\mathfrak{H}$ to all of $H$. Further the inequality (2.2.32) shows that the inverse map is continuous and this together with the closed graph theorem yields bicontinuity. Finally the lemma asserts that (2.2.33) is valid under this correspondence.

The full statement of our result contains a description of the adjoint system and we therefore proceed to the associated adjoint transformations. According to the general theory developed in $\$ 1.5$ the maximal and 
minimal adjoint transformations $\mathfrak{M}_{21}^{1}$ and $\mathfrak{M}_{21}^{0}$, respectively, are defined by the relations

$$
(2.2 .34) \quad \mathfrak{S}\left(\mathfrak{M}_{21}^{1}\right)=\mathfrak{U}\left[\mathfrak{S}\left(\mathfrak{R}_{21}^{1}\right)\right] \text { and } \quad \mathfrak{H}\left(\mathfrak{M}_{21}^{0}\right)=\mathfrak{U}\left[\mathfrak{H}\left(\mathfrak{R}_{21}^{0}\right)\right] \text {. }
$$

Consequently

$$
\mathfrak{M}_{21 z}^{1}{ }^{1}=\left[M_{21}^{1}{ }^{1}, \dot{B}_{21}^{*} \dot{z}^{1}-[C G]_{21} \dot{z}^{1}-C_{1 z}{ }^{i}\right]
$$

where $\mathfrak{D}\left(\mathfrak{M}_{21}^{1}\right)=\mathfrak{D}\left(\mathfrak{R}_{21}^{1}\right)$ and $z^{i}=\beta\left[z^{1}, L_{21}^{1} z^{1}\right]$. Furthermore $\mathfrak{M}_{21}^{0}$ is the restriction of $\mathfrak{M}_{21}^{1}$ with domain $\mathfrak{D}\left(\mathfrak{M}_{21}^{0}\right)=\mathfrak{D}\left(\mathfrak{R}_{21}^{0}\right)$ and hence can be represented simply as

$$
\mathfrak{M}_{21 z^{1}}^{0}=\left[M_{21}^{1} z^{1}, \dot{B}_{21}^{*} \dot{z}^{1}\right] .
$$

It is easy to see that the operator $\mathfrak{M}_{0}$ engendered by $\mathfrak{M}_{21}^{0}$ is

$$
\mathfrak{M}_{0 \dot{z}^{0}}=\left[M_{1} z^{0}, \dot{B}^{*} \dot{z}^{0}\right]
$$

where

$$
\mathfrak{D}\left(\mathfrak{M}_{0}\right)=\left[\left[z^{0}, \dot{z}^{0}\right] ; z^{0} \in \mathfrak{D}\left(M_{1}\right), \dot{z}^{0} \in \mathfrak{D}(G), z^{i}=-G \dot{z}^{0}\right]
$$

here again $z^{i}=\beta\left[I_{01}^{-1} z^{0}, L_{21}^{1} I_{01}^{-1} z^{0}\right]$. The operator $\mathfrak{M}_{1}$ engendered by $\mathfrak{M}_{21}^{1}$ requires a somewhat more complicated description. We have

$$
\mathfrak{M}_{1 z}^{0}=\left[M_{1} z^{0}, \dot{B}^{*} \dot{z}^{0}-\dot{I}_{20}^{-1}\left\{[C G]_{21} \dot{I}_{01}^{-1} \dot{z}^{0}+C_{1} z^{i}\right\}\right]
$$

where

$$
\begin{aligned}
\mathfrak{D}\left(\mathfrak{M}_{1}\right)=\left[\left[z^{0}, \dot{z}^{0}\right] ; z^{0} \in \mathfrak{D}\left(M_{1}\right), z^{i}\right. & \in \mathfrak{D}\left(C_{1}\right), \dot{z}^{0} \in \dot{I}_{01}\left[\dot{H}_{1}\right], \\
& \left.\left\{[C G]_{21} \dot{I}_{01}^{-1} \dot{z}^{0}+C_{1} z^{i}\right\} \in I_{20}\left[\dot{H}_{0}\right]\right] .
\end{aligned}
$$

By now we have not only verified the hypothesis of Theorem 1.5.1 but characterized the pertinent maximal and minimal operators as well. The conclusions of Theorems 1.5.1 and 1.6.1 are therefore valid and represent the principal result of this section. It will be noticed that the coupling transformation in the adjoint operator $\mathfrak{M}_{1}$ acts on the boundary space of $L_{21}^{1}$. By employing the transformation theory developed in $\$ 1.6$, this defect is easily rectified. In fact setting

$$
\boldsymbol{z}^{i^{\prime}}=U \boldsymbol{Z}^{i}, \quad C_{1}^{\prime}=C_{1} U^{\prime}, \text { and } G^{\prime}=U G,
$$

it is clear that $\mathfrak{M}_{0}$ and $\mathfrak{M}_{1}$ can be represented by the equivalent operators

$$
\begin{aligned}
\mathfrak{M}_{0}^{\prime} \mathfrak{\jmath}^{0} & =\left[M_{1} z^{0}, \dot{B}^{*} \dot{z}^{0}\right], \\
\mathfrak{D}\left(\mathfrak{M}_{0}^{\prime}\right) & =\left[\left[z^{0}, \dot{z}^{0}\right] ; z^{0} \in \mathfrak{D}\left(M_{1}\right), \dot{z}^{0} \in \mathfrak{D}\left(G^{\prime}\right), z^{i \prime}=-G^{\prime} \dot{z}^{0}\right]
\end{aligned}
$$

and 


$$
\mathfrak{M}_{1}^{\prime}{ }^{\theta}=\left[M_{1} z^{0}, \dot{B}^{*} \dot{z}^{0}-\dot{I}_{20}^{-1}\left\{[C G]_{21} \dot{I}_{01}^{-1} \dot{z}^{0}+C_{1}^{\prime} z^{i \prime}\right\}\right],
$$

$$
\begin{aligned}
\mathfrak{D}\left(\mathfrak{M}_{1}^{\prime}\right)=\left[\left[z^{0}, \dot{z}^{0}\right] ; z^{0} \in \mathfrak{D}\left(M_{1}\right), z^{i \prime} \in \mathfrak{D}\left(C_{1}^{\prime}\right), \dot{z}^{0} \in I_{01}\left[H_{1}\right],\right. \\
\left.\left\{[C G]_{21} \dot{I}_{01}^{-1} \dot{z}^{0}+C_{1}^{\prime} z^{i^{\prime}}\right\} \in \dot{I}_{20}\left[\dot{H}_{0}\right]\right] .
\end{aligned}
$$

It was shown in the proof of Theorem 1.6.1 for $z \in \mathcal{S}\left(M_{21}^{1}\right)$ that $\beta U z=\beta U_{0}^{\prime} \beta^{\prime} z$ or, equivalently, $\beta^{\prime} z=\beta^{\prime} U_{0} \beta U z$. Consequently $z^{i^{\prime}}=U z^{i}=\beta^{\prime} U_{0}[\beta U z]=\beta^{\prime} z$, that is

$$
z^{i \prime}=\beta^{\prime}\left[I_{01}^{-1} z^{0}, I_{20} M_{1}^{0} z\right] .
$$

When $C$ is bounded, the Corollary to Lemma 2.2 .5 permits us to state the boundary conditions on $\mathbb{R}$ and $\mathfrak{M}$ in terms of the $L_{1}$ and $M_{1}$ boundary spaces, namely $H$ and $H^{\prime}$ respectively. Thus in this case Theorem 1.5.1 takes the form: There is a one-to-one correspondence between the maximal negative subspaces $[N]$ of $H$, taken with respect to $\boldsymbol{Q}$, and the closed maximal dissipative operators $\mathfrak{R}$ on $H$ such that $\mathfrak{R}_{0} \subset \mathfrak{R} \subset \mathfrak{R}_{1}$, this correspondence being defined by

$$
\mathfrak{D}(\mathfrak{R})=\left[\mathfrak{y}^{0} ; \mathfrak{y}^{0} \in \mathfrak{D}\left(\mathfrak{R}_{1}\right), y^{e} \in N\right],
$$

where $y^{e}=y^{i}+G \dot{y}^{0}$. Moreover the adjoint operator $\mathfrak{M}=\Omega^{*}$ is again a maximal dissipative operator with dense domain, $\mathfrak{M}_{0} \subset \mathfrak{M} \subset \mathfrak{M}_{1}$, and

$$
\mathfrak{D}(\mathfrak{M})=\left[z^{0} ; z^{0} \in \mathfrak{D}\left(\mathfrak{M}_{1}\right), z^{e} \in P\right],
$$

where $\boldsymbol{z}^{e}=\boldsymbol{z}^{i}+G \dot{z}^{0}$ and $\boldsymbol{P}$ is the $\boldsymbol{Q}$-orthogonal complement of $\boldsymbol{N}$. As in the proof of Theorem 1.6.1, $z^{e} \in P$ if and only if

$$
\boldsymbol{z}^{e^{\prime}}=U \boldsymbol{z}^{e}=\boldsymbol{z}^{i^{\prime}}+G^{\prime} \dot{z}^{0}
$$

belongs to $U[P]$. Hence $(2.2 .46)$ is equivalent with

$$
\mathfrak{D}(\mathfrak{M})=\left[z^{0} ; z^{0} \in \mathfrak{D}\left(\mathfrak{M}_{1}\right), z^{e^{\prime}} \in U[P]\right] .
$$

We note that $U[P]$ is a maximal negative subspace of $\boldsymbol{H}^{\prime}$.

By way of illustrating the above development, we now sketch a treatment for the vibrating membrane on a unit disk. The equation of motion being

$$
u_{t t}=u_{x_{1} x_{1}}+u_{x_{2} x_{2}}, \quad \chi_{1}^{2}+\chi_{2}^{2}<1
$$

$$
t>0
$$

we set $u_{x_{1}}=\eta^{1}, u_{x_{2}}=\eta^{2}$, and $u_{t}=\eta^{3}$, and obtain the system (1.2) where

$$
A^{1}=\left(\begin{array}{lll}
0 & 0 & 1 \\
0 & 0 & 0 \\
1 & 0 & 0
\end{array}\right), \quad A^{2}=\left(\begin{array}{lll}
0 & 0 & 0 \\
0 & 0 & 1 \\
0 & 1 & 0
\end{array}\right), \quad E=I, \quad B=\Theta .
$$

It is clear that $D=\Theta$ so that $H_{1}=H_{0}=H_{2}=L_{2}(\Delta ; I), L_{0}=-M_{0}$, and $L_{1}$ $=-M_{1}$. According to (1.5.8) each element of the boundary space $H$ is 
represented by a solution of

$$
L_{1}^{2} y^{0}=y^{0},
$$

or, equivalently, by a weak solution of the system

$$
\begin{aligned}
& \stackrel{1}{\eta_{\chi_{1} x_{1}}}+\underset{\eta_{x_{1} x_{2}}}{2}=\eta^{1}, \\
& \stackrel{1}{\eta_{\chi_{1} x_{2}}}+\stackrel{\eta_{\chi_{2} \chi_{2}}^{2}}{\eta_{x_{1}}}=\eta^{2} \text {, } \\
& \stackrel{3}{\eta_{\chi_{1} x_{1}}}+\stackrel{3}{\eta_{\chi_{2} x_{2}}}=\eta^{3} \text {. }
\end{aligned}
$$

It can be shown that weak solutions correspond to regular solutions for such a system and further that the first two equations are equivalent to

$$
\eta^{1}=\phi_{\chi_{1}}, \quad \eta^{2}=\phi_{\chi_{2}}, \quad \phi_{\chi_{1} x_{1}}+\phi_{x_{2} x_{2}}=\phi .
$$

Representing the boundary values of $\eta^{3}$ and $\partial \phi / \partial r$ (here $r^{2}=\chi_{1}^{2}+\chi_{2}^{2}$ ) by Fourier series

$$
\text { (2.2.52) } \quad \eta^{3} \sim(2 \pi)^{-1 / 2} \sum_{k=-\infty}^{\infty} a_{k} e^{i k \sigma}, \quad \partial \phi / \partial r \sim(2 \pi)^{-1 / 2} \sum_{k=-\infty}^{\infty} b_{k} e^{i k \sigma},
$$

the Bessel function expansions for $\eta^{3}$ and $\phi$ are simply

$$
\begin{aligned}
\eta^{3}(r, \sigma) & =(2 \pi)^{-1 / 2} \sum_{k=-\infty}^{\infty} a_{k}\left\{I_{k}(1)\right\}^{-1} I_{k}(r) e^{i k \sigma} \\
\phi(r, \sigma) & =(2 \pi)^{-1 / 2} \sum_{k=-\infty}^{\infty} b_{k}\left\{I_{k}^{\prime}(1)\right\}^{-1} I_{k}(r) e^{i k \sigma} ;
\end{aligned}
$$

and the norm of $y=\left[\eta^{3}, \partial \phi / \partial r\right]$ is given by

$$
\begin{aligned}
\|y\|^{2} & =\left\langle y^{0}, y^{0}\right\rangle+\left\langle L_{1} y^{0}, L_{1} y^{0}\right\rangle=\int_{\Gamma} \eta^{3}\left(\partial \eta^{3} / \partial r\right)^{-}+\phi(\partial \phi / \partial r)-d \sigma \\
& =\sum_{k=-\infty}^{\infty}\left(\rho_{k}\left|a_{k}\right|^{2}+\rho_{k}^{-1}\left|b_{k}\right|^{2}\right),
\end{aligned}
$$

where $\rho_{\boldsymbol{k}}=I_{k}^{\prime}(1) / I_{k}(1)$. It follows that the boundary space $H$ is the direct product of hilbert spaces $h_{1}$ and $h_{2}$ with elements $\boldsymbol{n}^{1}=\eta^{3}$ and $\mathbf{n}^{2}=\partial \phi / \partial r$, and norms

$$
\left\|\mathbf{n}^{1}\right\|_{1}^{2}=\sum \rho_{k}\left|a_{k}\right|^{2} \text { and }\left\|\mathbf{n}^{2}\right\|_{2}^{2}=\sum \rho_{k}^{-1}\left|b_{k}\right|^{2} \text {, }
$$

respectively. These are dual spaces and the transformation

$$
f_{12}\left\{b_{k}\right\}=\left\{\rho_{k}^{-1} b_{k}\right\}
$$

on $\boldsymbol{h}_{2}$ to $\boldsymbol{h}_{1}$ is unitary with inverse $\boldsymbol{f}_{21}\left\{a_{k}\right\}=\left\{\rho_{k} a_{k}\right\}$. 
In the present example the third relation in (1.3.4) becomes $W\left[y^{1}, y^{2}\right]$ $=\left[y^{2}, y^{1}\right]$. We also recall that $y=\left[y^{1}, L_{1} y^{1}\right] \in N_{1} \cap N_{0}^{\perp}$ if and only if $L_{1}^{2} y^{1}=y^{1}$. On the other hand for such a $y, W y=\left[L_{1} y^{1}, y^{1}\right]=\left[L_{1} y^{1}, L_{1}\left[L_{1} y^{1}\right]\right]$ and $\left(L_{1}\right)^{2} L_{1} y^{1}=L_{1}\left[L_{1}^{2} y^{1}\right]=L_{1} y^{1}$ so that $W$ maps $N_{1} \cap N_{0}^{\perp}$ on itself. Moreover in going from $y^{1}$ to $L_{1} y^{1}$ we see that $\eta^{1} \rightarrow \eta_{x_{1}}^{3}, \eta^{2} \rightarrow \eta_{x_{2}}^{3}$, and $\eta^{3} \rightarrow \eta_{x_{1}}^{1}+\eta_{x_{2}}^{2}=\Delta \phi=\phi$. Hence $W\left[\eta^{3}, \partial \phi / \partial r\right]=\left[\phi, \partial \eta^{3} / \partial r\right]$. In other words

$$
W\left[\mathbf{n}^{1}, \mathfrak{n}^{2}\right]=\left[\boldsymbol{f}_{12} \mathbf{n}^{2}, \boldsymbol{f}_{21} \mathbf{n}^{1}\right] .
$$

Finally the relevant quadratic form on $H$ is

$$
\begin{aligned}
Q(y, y) & =\int_{\Gamma}\left\{\eta^{3}(\partial \phi / \partial r)^{-}+\left[\eta^{3}\right]-\partial \phi / \partial r\right\} d \sigma=\left\langle\mathbf{n}^{1}, \mathbf{n}^{2}\right\rangle+\left\langle\mathbf{n}^{2}, \mathbf{n}^{1}\right\rangle \\
& =\frac{1}{2}\left\{\left\|\mathbf{n}^{1}+\boldsymbol{f}_{12} \mathbf{n}^{2}\right\|_{1}^{2}-\left\|\mathbf{n}^{1}-\boldsymbol{f}_{12} \mathbf{n}^{2}\right\|_{1}^{2}\right\},
\end{aligned}
$$

and the general boundary condition given by a maximal negative subspace $N$ of $H$ is characterized by an arbitrary contraction operator $J$ on $h_{1}$ to itself as

$$
\mathbf{n}^{1}+f_{12} \mathbf{n}^{2}+J\left[\mathbf{n}^{1}-f_{12} \mathbf{n}^{2}\right] .
$$

We next impose an elastic type boundary condition on the membrane by adjoining to the membrane system the equation

$$
\dot{\eta}_{t}=\kappa \boldsymbol{n}^{1}
$$

which governs the boundary system; here $\kappa$ is a multiplicative operator on $h_{1}$ (considered as a space of functions on the circle $\Gamma$ ) to $\dot{H}_{0}=L_{2}(\Gamma)$. Assuming the multiplier $\kappa(\cdot)$ to be measurable and bounded, it is easy to see that $\kappa$ is a bounded linear transformation. Further

$$
\dot{B}=\Theta \quad \text { and } \quad C=\left(\begin{array}{ll}
\kappa & \Theta
\end{array}\right) .
$$

Now $\left\langle\boldsymbol{k} \mathbf{n}^{1}, \dot{\eta}\right\rangle=\left\langle\mathbf{n}^{1}, \boldsymbol{f}_{12} \kappa_{1} \dot{\eta}\right\rangle_{1}$, where $\kappa_{1}$ is the bounded multiplicative transformation on $\dot{H}_{0}$ to $h_{2}$ defined by the function $[\kappa(\cdot)]$-. It follows that

$$
C^{*}=\left(\begin{array}{c}
f_{12} \kappa_{1} \\
\Theta
\end{array}\right) \text { and } G=W C^{*}=\left(\begin{array}{c}
\Theta \\
\kappa_{1}
\end{array}\right) \text {. }
$$

The transformations $C$ and $G$ being bounded, we conclude that the mapping $\mathfrak{y} \rightarrow y^{e}=y^{i}+G \dot{y}^{0}$ is an isomorphism of $\mathfrak{H}$ onto $\boldsymbol{H}$ under which $\boldsymbol{Q}\left(\boldsymbol{y}^{e}, \boldsymbol{y}^{e}\right)$ $=\mathfrak{Q}(\mathfrak{y}, \mathfrak{y})$. In this case

$$
y^{e}=\left[\mathbf{n}^{1}, \mathbf{n}^{2}+\kappa_{1} \dot{\eta}\right] .
$$

The maximal negative subspaces of $\mathfrak{F}$ are defined by contraction operators $J$ as in (2.2.59) and in particular $J=I$ gives $\left(y^{e}\right)^{2}=\mathbf{n}^{2}+\kappa_{1} \dot{\eta}=\theta$ as a boundary condition. On the other hand, $\mathbf{n}^{2}=\partial u /\left.\partial r\right|_{r=1}$ and by (2.2.60) $\dot{\eta}_{t}=\kappa \mathbf{n}^{1}=\left.\kappa u_{t}\right|_{r=1}$ 
so that we have in essence

$$
\partial u /\left.\partial r\right|_{r=1}+\left.\kappa_{1} \kappa u\right|_{r=1}=0
$$

the familiar boundary condition associated with an elastic constraint on the boundary.

A somewhat more interesting boundary problem is obtained when we couple a spring with mass attached to the membrane in which case we require an unbounded coupling operator. The boundary space is now $\dot{H}_{0}=L_{2}(\Gamma$ ) $\times L_{2}(\Gamma)$ and the boundary system is governed by the equations

$$
\begin{aligned}
& \dot{\eta}_{t}^{1}=\mu \dot{\eta}^{2} \\
& \dot{\eta}_{t}^{2}=-\mu \dot{\eta}^{1}-\nu^{-1} \mathbf{n}^{2}
\end{aligned}
$$

where $\mu$ and $\nu$ are multiplicative operators corresponding to real measurable bounded functions $\mu(\cdot)$ and $\nu(\cdot)$, in fact we even assume that $\nu(\cdot)$ is bounded away from zero. As such $\mu$ is clearly a bounded operator on $L_{2}(\Gamma)$ to itself. On the other hand $\nu$ is bounded on $L_{2}(\Gamma)$ to $h_{2}$ and hence $\nu^{-1}$ is closed on $h_{2}$ to $L_{2}(\Gamma)$ with a dense domain consisting of those functions whose Fourier coefficients are square summable (without the $\rho_{k}^{-1}$ factor). We now have

$$
B=\left(\begin{array}{cc}
\Theta & \mu \\
-\mu & \Theta
\end{array}\right) \text { and } C=\left(\begin{array}{cc}
\Theta & \Theta \\
\Theta \mathbf{1} & -\nu^{-1}
\end{array}\right) \text {. }
$$

Again $\left\langle\nu \dot{\eta}^{2}, \mathbf{n}^{2}\right\rangle_{2}=\left\langle\dot{\eta}^{2}, \nu_{1} \boldsymbol{f}_{12} \mathbf{n}^{2}\right\rangle$ where $\nu_{1}$ is the bounded linear multiplicative operator on $h_{1}$ to $L_{2}(\Gamma)$ defined by $\nu(\cdot)$. Thus $\left(\nu^{-1}\right)^{*}=\left(\nu^{*}\right)^{-1}=f_{21} \nu_{1}^{-1}$ and

$$
C^{*}=\left(\begin{array}{cc}
\Theta & \Theta \\
\Theta & -f_{21} \nu_{1}^{-1}
\end{array}\right) \text { and } \quad G=W C^{*}=\left(\begin{array}{cc}
\Theta & -\nu_{1}^{-1} \\
\Theta & \Theta
\end{array}\right) \text {. }
$$

Finally we note that $C W C^{*}=\Theta$, with domain $\mathfrak{D}\left(C^{*}\right)$, and this together with the fact that $F=I$ shows that Condition (i) is satisfied. Likewise (ii) is satisfied since $\dot{B}+\dot{B}^{*}-C W C^{*}=\Theta$ and we see, incidentally, that $\dot{H}_{1}=\dot{H}_{0}=\dot{H}_{2}$.

The boundary space elements are represented in the present instance by solutions of $\mathfrak{M}_{1} \mathfrak{R}_{1} \mathfrak{y}^{1}=-\mathfrak{y}^{1}$ or, equivalently, by solutions of (2.2.50) plus

$$
B^{*}\left[B \dot{y}^{1}+C y^{i}\right]-C z^{i}=-\dot{y}^{1},
$$

where $y^{i}=\beta\left[y^{1}, L_{1} y^{1}\right]=\left[\eta^{3}, \partial \phi / \partial r\right]$ and $z^{i}=\beta\left[L_{1} y^{1}, y^{1}\right]=\left[\phi, \partial \eta^{3} / \partial r\right]$. The Equations (2.2.50) are solved as before and, in terms of the functions $\eta^{3}$ and $\phi$, we obtain as the solution of (2.2.68)

$$
\begin{aligned}
& \dot{\eta}^{1}=-\mu\left(1+\mu^{2}\right)^{-1} \nu^{-1} \partial \phi / \partial r \\
& \dot{\eta}^{2}=-\left(1+\mu^{2}\right)^{-1} \nu^{-1} \partial \eta^{3} / \partial r .
\end{aligned}
$$

From this one readily computes for $\mathfrak{y}=\left[\mathfrak{y}^{1}, \mathfrak{l}_{1} \mathfrak{y}^{1}\right]$ 


$$
\begin{aligned}
\|\mathfrak{y}\|^{2}= & \int_{\Gamma}\left\{\eta^{3}\left(\partial \eta^{3} / \partial r\right)^{-}+\phi(\partial \phi / \partial r)^{-}\right. \\
& \left.+\left(1+\mu^{2}\right)^{-1}\left(\left|\nu^{-1} \partial \eta^{3} / \partial r\right|^{2}+\left|\nu^{-1} \partial \phi / \partial r\right|^{2}\right)\right\} d \sigma,
\end{aligned}
$$

and

$$
\mathfrak{Q}(\mathfrak{y}, \mathfrak{y})=2 \operatorname{Re}\left[\int_{\Gamma}\left\{\nu_{1} \eta^{3}+\left(1+\mu^{2}\right)^{-1} \nu^{-1} \partial \eta^{3} / \partial r\right\}\left\{\nu^{-1} \partial \phi / \partial r\right\}-d \sigma\right] .
$$

Finally since $\eta^{3}$ and $\phi$ are mutually independent and since the range of $\nu^{-1}$ is $L_{2}(\Gamma)$, we see that a maximal negative subspace is determined by the relation $\nu_{1} \eta^{3}+\left(1+\mu^{2}\right)^{-1} \nu^{-1} \partial \eta^{3} / \partial r=0$ on $\Gamma$, in other words $\dot{\eta}^{2}=\nu_{1} \eta^{3}$ on $\Gamma$. Now by virtue of $(2.2 .65)$ we have $\left.\mu \nu_{1} u_{t}\right|_{r=1}=\mu \nu_{1} \eta^{3}=\dot{\eta}_{t}^{1}$. In essence then $\dot{\eta}^{1}=\left.\mu \nu_{1} u\right|_{r=1}$ and hence by (2.2.65)

$$
\left.\nu_{1} u_{t t}\right|_{r=1}+\left.\mu^{2} \nu_{1} u\right|_{r=1}=-\nu^{-1} \partial u /\left.\partial r\right|_{r=1},
$$

which is the boundary condition associated with a spring plus mass constraint at the boundary.

\section{APPENDIX}

2.3. General maximal dissipative extensions of $L_{0}$ and restrictions of $L_{1}$. The body of this chapter has dealt with maximal and properly dissipative extensions of $L_{0}$, that is, maximal dissipative extensions of $L_{0}$ which are at the same time restrictions of $L_{1}$. If $F=I$, then $L_{0}$ is conservative, $L_{1}=-L_{0}^{*}$, and we see from Lemma 1.1.5 that the properly dissipative operators are the only maximal dissipative extensions of $L_{0}$. However in the general case, $F \neq I$, other maximal dissipative extensions of $L_{0}$ exist and, dually, there are maximal dissipative restrictions of $L_{1}$ whose domains do not contain $\mathfrak{D}\left(L_{0}\right)\left({ }^{7}\right)$. Such extensions of $L_{0}$ need not be differential operators; the physical models associated with operators of this kind allow for part of the energy which leaves the system through the boundary to be redistributed in the interior $\left(^{3}\right)$. The adjoint $M$ of such an operator is a restriction of $M_{1}$ with $\mathfrak{D}(M) D \mathfrak{D}\left(M_{0}\right)$ and, in the associated model, energy may enter through the boundary, but only to an extent which can be compensated for by internal energy losses. In this section we shall make an exploratory study of these nonproperlydissipative operators; however, the problem of determining the most general maximal dissipative extension of $L_{0}$ or restriction of $L_{1}$ is left open.

We shall assume that $L$ is a maximal dissipative extension of $L_{0}$ with domain contained in $\mathfrak{D}\left(L_{1}\right)$. In this case $L-L_{1}$ annihilates $\mathfrak{D}\left(L_{0}\right)$ and hence $\left(L-L_{1}\right) y^{0}$ depends only on the coset of $\mathfrak{D}(L) / \mathfrak{D}\left(L_{0}\right)$ to which $y^{0}$ belongs. Now

$$
\mathfrak{D}(L) / \mathfrak{D}\left(L_{0}\right)
$$

is isomorphic to a subspace $N$ of $H$ under the mapping 


$$
\gamma: y^{0} \rightarrow y=\beta\left[I_{01}^{-1} y^{0}, L_{21}^{1} I_{01}^{-1} y^{0}\right] .
$$

Thus $L-L_{1}$ defines a linear transformation $T$ on $N$ to $H_{0}$ such that

$$
L y^{0}=L_{1} y^{0}+T \gamma y^{0},
$$

We shall soon restrict $T$ further; however first it will be instructive to deduce certain properties of $T$ and $N$ implied by the above basic assumption.

Lemma 2.3.1. $\boldsymbol{N}$ is a negative subspace of $\boldsymbol{H}$.

Proof. Suppose there were a $y^{0} \in \mathfrak{D}(L)$ such that $Q\left(\gamma y^{0}, \gamma y^{0}\right)>0$. Then since $\mathfrak{D}\left(I_{20} L_{0} I_{01}\right)$ is dense in $H_{1}$, we can choose a sequence $\left\{u_{n}^{0}\right\} \subset \mathfrak{D}\left(L_{0}\right)$ with the property $I_{01}^{-1} u_{n}^{0} \rightarrow I_{01}^{-1} y^{0}$ in $H_{1}$. Setting $w_{n}^{0}=y^{0}-u_{n}^{0}$, we see that $\left\{w_{n}^{0}\right\} \subset \mathfrak{D}(L)$, $\gamma w_{n}^{0}=\gamma y^{0}, I_{01}^{-1} w_{n}^{0} \rightarrow \theta$ in $H_{1}$, and $w_{n}^{0} \rightarrow \theta$ in $H_{0}$. Consequently $\left(^{8}\right)$

$$
\begin{aligned}
\left\langle L w_{n}^{0}, w_{n}^{0}\right\rangle+\left\langle w_{n}^{0}, L w_{n}^{0}\right\rangle= & Q\left(\gamma y^{0}, \gamma y^{0}\right)+\left\langle D w_{n}^{0}, w_{n}^{0}\right\rangle \\
& +\left\langle T \gamma y^{0}, w_{n}^{0}\right\rangle+\left\langle w_{n}^{0}, T \gamma y^{0}\right\rangle
\end{aligned}
$$

and this converges to $Q\left(\gamma y^{0}, \gamma y^{0}\right)>0$ as $n \rightarrow \infty$. However this is contrary to $L$ being dissipative.

Since $\boldsymbol{Q}\left(\gamma y^{0}, \gamma y^{0}\right)$ is a measure of the rate at which energy enters through the boundary, the above lemma shows for the model associated with $L$ that energy does not enter through the boundary. On the other hand, $T$ has the effect of channeling energy directly into the interior at a rate depending on the boundary data.

Lemma 2.3.2. If $\Re(T) \subset I_{01}\left[H_{1}\right]$, then $T y=\theta$ whenever $y \in N$ and $\boldsymbol{Q}(y, y)$ $=0$.

Proof. If the assertion were false, there would exist a $y^{0} \in \mathfrak{D}(L)$ such that $\boldsymbol{Q}\left(\gamma y^{0}, \gamma y^{0}\right)=0$ and $T \gamma y^{0} \neq \theta$. For a given $\epsilon>0$, choose a sequence $\left\{u_{n}^{0}\right\}$ $\subset \mathfrak{D}\left(L_{0}\right)$ with the property $I_{01}^{-1} u_{n}^{0} \rightarrow I_{01}^{-1}\left(\epsilon T \gamma y^{0}-y^{0}\right)$. Setting $w_{n}^{0}=y^{0}+u_{n}^{0}$ we see that $\left\{w_{n}^{0}\right\} \subset \mathfrak{D}(L), \gamma w_{n}^{0}=\gamma y^{0}, I_{01}^{-1} w_{n}^{0} \rightarrow I_{01}^{-1}\left(\epsilon T \gamma y^{0}\right)$ in $H_{1}$, and $w_{n}^{0} \rightarrow \epsilon T \gamma y^{0}$ in $H_{0}$. Thus (2.3.3) holds and

$$
\left\langle L w_{n}^{0}, w_{n}^{0}\right\rangle+\left\langle w_{n}^{0}, L w_{n}^{0}\right\rangle \rightarrow \epsilon^{2}\left\langle D T \gamma y^{0}, T \gamma y^{0}\right\rangle+2 \epsilon\left\|T_{\gamma} y^{0}\right\|^{2},
$$

which is positive for a sufficiently small choice of $\epsilon$. Again this is contrary to $L$ being dissipative.

The physical interpretation of Lemma 2.3.2 is that $T$ transports energy into the interior only when energy leaves through the boundary. This is reasonable enough and suggests that it would be better to define $T$ as a transformation on the quotient space

$\left.{ }^{8}\right)$ Here we use the notation $\left\langle D w^{0}, z^{0}\right\rangle \equiv \int_{\Delta}\left(E D w^{0}, z^{0}\right) d \chi=\left\langle D_{21} I_{01}^{-1} w^{0}, I_{01}^{-1} z^{0}\right\rangle$, valid for all $w^{0}, z^{0} \in I_{01}\left[H_{1}\right]$. 


$$
\widehat{N}_{o} \equiv N / N_{o}
$$

where

$$
N_{0}=[y ; Q(y, y)=0, y \in N] .
$$

According to Lemma 2.3.1, $-\boldsymbol{Q}$ is a positive hermitian bilinear form on $N$. It follows that $-\boldsymbol{Q}$ defines a positive definite bilinear form on $\widehat{N}_{o}$, namely $-\widehat{\boldsymbol{Q}}$, where we set $\widehat{\boldsymbol{Q}}(\widehat{\boldsymbol{y}}, \widehat{\boldsymbol{z}})=\boldsymbol{Q}(\boldsymbol{y}, \boldsymbol{z})$ for any $\boldsymbol{y} \in \widehat{y}$ and $\boldsymbol{z} \in \widehat{z}$. Letting

$$
\langle\widehat{y}, \widehat{z}\rangle=-\widehat{Q}(\widehat{y}, \widehat{z}),
$$

$\widehat{N}_{o}$ becomes a pre-hilbert space whose completion we denote by $\widehat{N}$. We shall denote the mapping which sends $y^{0} \in \mathfrak{D}(L)$ into the coset $\widehat{y}$ containing $\gamma y^{0}$ by the symbol $\hat{\gamma}$.

With this preliminary discussion as motivation, we now suppose that $L$ is of the form

$$
L y^{0}=L_{1} y^{0}+\hat{T} \hat{\gamma} y^{0}
$$$$
y^{0} \in \mathfrak{D}(L),
$$

where $\hat{T}$ is a linear bounded transformation on $\widehat{N}$ to $H_{0}$. This amounts to a rather strong assumption on $T$ and implies, for instance, that the adjoint transformation $\hat{T}^{*}$ on $H_{0}$ to $\widehat{N}$ exists. Thus for $y^{0} \in \mathcal{D}(L)$ and $z^{0} \in H_{0}$ we have

$$
\left\langle\hat{T} \hat{\gamma} y^{0}, z^{0}\right\rangle=\left\langle\hat{\gamma} y^{0}, \hat{T}^{*} z^{0}\right\rangle=-\widehat{Q}\left(\hat{\gamma} y^{0}, \hat{T}^{*} z^{0}\right) .
$$

Consequently for $y^{0}, z^{0} \in \mathfrak{D}(L)$ we can write

$$
\begin{aligned}
\left\langle L y^{0}, z^{0}\right\rangle+ & \left\langle y^{0}, L z^{0}\right\rangle \\
& =\widehat{Q}\left(\hat{\gamma} y^{0}, \hat{\gamma} z^{0}\right)+\left\langle D y^{0}, z^{0}\right\rangle-\widehat{Q}\left(\hat{\gamma} y^{0}, \hat{T}^{*} z^{0}\right)-\widehat{Q}\left(\hat{T}^{*} y^{0}, \hat{\gamma} z^{0}\right) \\
& =\widehat{Q}\left(\hat{\gamma} y^{0}-\hat{T}^{*} y^{0}, \hat{\gamma} z^{0}-\hat{T}^{*} z^{0}\right)+\left\langle\left(D+\hat{T} \hat{T}^{*}\right) y^{0}, z^{0}\right\rangle ;
\end{aligned}
$$

here we have used the identity $\widehat{\boldsymbol{Q}}\left(\hat{T}^{*} y^{0}, \hat{T}^{*} z^{0}\right)=-\left\langle\hat{T} \hat{T}^{*} y^{0}, z^{0}\right\rangle$.

Lemma 2.3.3. Considered as a quadratic form on $I_{01}\left[H_{1}\right]$,

$$
D+\hat{T} \hat{T} \leqq \Theta .
$$

Proof. Suppose the contrary were the case. Then since $I_{01}^{-1}\left[\mathfrak{D}\left(L_{0}\right)\right]$ is dense in $H_{1}$, there is a $y^{0} \in \mathfrak{D}\left(L_{0}\right)$ such that

$$
\left\langle\left(D+\hat{T} \hat{T}^{*}\right) y^{0}, y^{0}\right\rangle>0 .
$$

Further, since $\hat{T}^{*} y^{0} \in \widehat{N}$ and $\hat{\gamma}[\mathfrak{D}(L)]$ is dense in $\widehat{\boldsymbol{N}}$ (by construction), we can find a sequence $\left\{u_{n}^{0}\right\} \subset \mathfrak{I}(L)$ such that $\hat{\gamma} u_{n}^{0} \rightarrow \hat{T}^{*} y^{0}$ in $\widehat{N}$ and again making use of the fact that $I_{01}^{-1}\left[\mathfrak{D}\left(L_{0}\right)\right]$ is dense in $H_{1}$, we may further suppose that $I_{01}^{-1} u_{n}^{0} \rightarrow \theta$ in $H_{1}$. Then

$$
w_{n}^{0}=y^{0}+u_{n}^{0} \in \mathfrak{D}(L), \quad \hat{\gamma} w_{n}^{0}=\hat{\gamma} u_{n}^{0} \rightarrow \hat{T}^{*} y^{0}, \quad I_{01}^{-1} w_{n}^{0} \rightarrow I_{01}^{-1} y^{0} \text { in } H_{1},
$$

and 


$$
w_{n}^{0} \rightarrow y^{0} \text { in } H_{0} .
$$

It now follows from (2.3.9) that

$$
\left\langle L w_{n}^{0}, w_{n}^{0}\right\rangle+\left\langle w_{n}^{0}, L w_{n}^{0}\right\rangle \rightarrow\left\langle\left(D+\hat{T} \hat{T}^{*}\right) y^{0}, y^{0}\right\rangle>0,
$$

which is impossible for $L$ dissipative.

Lemma 2.3.4. Let $\boldsymbol{N}^{-}$denote the closure of $\boldsymbol{N}=\gamma[\mathfrak{D}(L)]$. Then $\boldsymbol{N}^{-}$is a maximal negative subspace of $\boldsymbol{H}$ and

$$
\mathfrak{D}(L)=\left[y^{0} ; y^{0} \in \mathfrak{D}\left(L_{1}\right) \text { and } \gamma y^{0} \in N^{-}\right] .
$$

Proof. By assumption $\mathfrak{D}\left(L_{0}\right) \subset \mathfrak{D}(L) \subset \mathfrak{D}\left(L_{1}\right)$, and it follows from this fact alone that $\mathfrak{D}(L)$ is of the form (2.3.11) with $\boldsymbol{N}^{-}$replaced by $\boldsymbol{N}=\gamma[\mathfrak{D}(L)]$. According to Lemma 2.3.1, $N$ is a negative subspace of $H$. Hence if $N$ is not maximal negative, it is certainly contained in some maximal negative subspace, say $\boldsymbol{N}^{-}$. We set

$$
\mathfrak{D}^{-}=\left[y^{0} ; y^{0} \in \mathfrak{D}\left(L_{1}\right) \text { and } \gamma y^{0} \in N^{-}\right]
$$

and show that $\mathfrak{D}(L)=\mathfrak{D}^{-}$. Obviously $\mathfrak{D}(L) \subset \mathfrak{D}^{-}$. Again defining

$$
N_{0}^{-}=\left[y ; Q(y, y)=0, y \in N^{-}\right],
$$

we proceed as before and construct the pre-hilbert space

$$
\widehat{N}_{o}^{-}=N^{-} / N_{0}^{-}
$$

of residue classes $\widehat{y}^{-}, \widehat{z}^{-}$having as inner produce $\left\langle\hat{y}^{-}, \hat{z}^{-}\right\rangle=-\widehat{Q}^{-}\left(\hat{\boldsymbol{y}}^{-}, \widehat{z}^{-}\right)$, where $\widehat{Q}^{-}\left(\hat{y}^{-}, \widehat{z}^{-}\right)=Q(y, z)$ for all $y \in \hat{y}^{-}$and $z \in \widehat{z}^{-}$. Likewise we denote the mapping which takes $y^{0} \in \mathfrak{D}^{-}$into the coset $\hat{y}^{-}$containing $\gamma y^{0}$ by the symbol $\hat{\gamma}^{-}$. It is clear that $N_{0}=N_{0}^{-} \cap N$ and it follows from this that $\widehat{N}_{0}$ can be embedded in $\widehat{N}_{o}^{-}$under the mapping which takes $\widehat{y}$ into $\widehat{y}^{-}$if the residue class $\widehat{y}$ is contained in the residue class $\widehat{y}^{-}$; in particular, the inner product is preserved under this mapping. Moreover, $\hat{\gamma}^{-}$is in this sense an extension of $\hat{\gamma}$. Finally, if $\widehat{N}^{-}$denotes the completion of $\widehat{N}_{o}^{-}$, then it is clear that $\widehat{N}$ can be thought of as a closed subspace of $\widehat{N}^{-}$, and that $\hat{T}$ can be thought of as a transformation of this subspace to $H_{0}$. We note that each $\widehat{y}^{-} \in \widehat{N}^{-}$has a unique representation $\widehat{y}^{-}=\widehat{y}_{1}^{-}+\widehat{y}_{2}^{-}$, where $\widehat{y}_{1}^{-} \in \widehat{N}$ and $\widehat{y}_{2}^{-} \in \widehat{N}^{-} \cap N^{\perp}$. We now extend $\hat{T}$ onto $\widehat{N}^{-}$by setting

$$
\hat{T}^{-} \widehat{y}^{-}=\hat{T} \widehat{y}_{\overline{1}}^{-} .
$$

Then $\hat{T}^{-}$is a bounded linear transformation on $\widehat{N}^{-}$to $H_{0}$. Next we define what is clearly an extension of $L$, namely,

$$
L^{-} y^{0}=L_{1} y^{0}+\hat{T}^{-} \hat{\gamma}^{-} y^{0}, \quad y^{0} \in \mathfrak{D}^{-},
$$

and we show that $L^{-}$is dissipative. In fact, suppose $y^{0} \in \mathfrak{D}^{-}$and let $\hat{\gamma}^{-} y^{0}$ $=\widehat{y}^{-}=\widehat{y}_{1}^{-}+\widehat{y}_{2}^{-}$. Then 


$$
\widehat{Q}^{-}\left(\widehat{y}^{-}, \widehat{y}^{-}\right)=\widehat{Q}\left(\widehat{y}_{1}^{-}, \widehat{y}_{1}^{-}\right)+\widehat{Q}^{-}\left(\hat{y}_{2}^{-}, \widehat{y}_{2}^{-}\right)
$$

and

$$
\left\langle\hat{T}^{-} \widehat{y}^{-}, y^{0}\right\rangle=\left\langle\hat{T}_{y_{1}^{-}}, y^{0}\right\rangle=\left\langle\widehat{y}_{\overline{1}}^{-}, \hat{T}^{*} y^{0}\right\rangle=-\widehat{Q}\left(\widehat{y}_{1}^{-}, \hat{T}^{*} y^{0}\right) .
$$

Consequently, we obtain in analogy to (2.3.9)

$$
\begin{aligned}
\left\langle L^{-} y^{0}, y^{0}\right\rangle+ & \left\langle y^{0}, L^{-} y^{0}\right\rangle \\
& =\widehat{Q}^{-}\left(\widehat{y}_{2}^{-}, \widehat{y}_{2}^{-}\right)+\widehat{Q}\left(\widehat{y}_{1}^{-}-\hat{T}^{*} y^{0}, \widehat{y}_{1}^{-}-\hat{T}^{*} y^{0}\right)+\left\langle\left(D+\hat{T} \hat{T}^{*}\right) y^{0}, y^{0}\right\rangle
\end{aligned}
$$

which is $\leqq 0$ since each term in the right member is nonpositive, the last being so by Lemma 2.3.3. Thus $L^{-}$is dissipative and it follows from the fact that $L$ was assumed to be maximal dissipative that $L=L^{-}$and hence that $\mathfrak{D}(L)=\mathfrak{D}^{-}$. Thus $\mathfrak{D}(L)$ is of the type considered in Theorem 1.5.1 and hence there is a maximal dissipative operator $L^{\prime}$ such that $\mathfrak{D}(L)=\mathfrak{D}\left(L^{\prime}\right)$ and $L_{0} \subset L^{\prime} \subset L_{1}$. However, $L^{\prime}$ is engendered from a transformation $L_{21}^{\prime}$ for which $\beta\left[\oiint\left(L_{21}^{\prime}\right)\right]=N^{-}$and, according to Theorem 1.3.1, $\oiint\left(I_{20} L^{\prime} I_{01}\right)$ is dense in (S) $\left(L_{21}^{\prime}\right)$. It follows that $\gamma[D(L)]=\beta\left[\mathfrak{G}\left(I_{20} L^{\prime} I_{01}\right)\right]$ is dense in $\boldsymbol{N}^{-}$. Incidently, this shows that $\widehat{N}_{0}$ is dense in $\widehat{N}_{0}^{-}$and hence that $\widehat{N}=\widehat{N}^{-}$.

We summarize these results in

THEOREM 2.3.1. Let $L$ be a maximal dissipative extension of $L_{0}$ with $\mathfrak{D}(L)$ $\subset \mathfrak{D}\left(L_{1}\right)$. Then $N=\gamma[\mathfrak{D}(L)]$ is a negative subspace of $H$ and we can construct the hilbert space $\widehat{N}$ from the pre-hilbert space defined by (2.3.4) and (2.3.6). Suppose further that $L$ is of the form (2.3.7), where $\hat{T}$ is a bounded linear transformation on $\widehat{N}$ to $H_{0}$. Then $\hat{T}$ necessarily satisfies the Condition (2.3.10), the closure of $N$, namely $N^{-}$, is a maximal negative subspace of $H$ defining the same hilbert space $\widehat{N}$ as $N$, and $\mathfrak{D}(L)$ is given by (2.3.11).

In the converse direction we have

Theorem 2.3.2. Suppose $\boldsymbol{N}$ is a maximal negative subspace of $\boldsymbol{H}$ and construct the hilbert space $\widehat{N}$ from the pre-hilbert space defined by (2.3.4) and (2.3.6). Let $\hat{T}$ be a bounded linear transformation on $\widehat{N}$ to $H_{0}$ satisfying the Condition (2.3.10). Then

$$
\begin{aligned}
L y^{0} & =L_{1} y^{0}+\hat{T} \hat{\gamma} y^{0}, \\
\mathfrak{D}(L) & =\left[y^{0} ; y^{0} \in \mathfrak{D}\left(L_{1}\right) \text { and } \gamma y^{0} \in N\right]
\end{aligned}
$$

defines a maximal dissipative extension of $L_{0}$.

Proof. Since $\hat{\gamma}\left[\mathfrak{D}\left(L_{0}\right)\right]=\widehat{\boldsymbol{\theta}}$, it is clear that $L \supset L_{0}$ and it follows from (2.3.9) that $L$ is dissipative. It remains, therefore, to show that $L$ is maximal dissipative and for this it suffices to prove that $\Re(\lambda I-L)=H_{0}$ for some $\lambda>0$, according to the corollary to Theorem 1.1.1. We shall establish this fact with the help of the maximal dissipative operator $L^{\prime}$ defined in Theorem 1.5.1 with $\mathfrak{D}\left(L^{\prime}\right)=\mathfrak{D}(L)$ and $L_{0} \subset L^{\prime} \subset L_{1}$. We show, first of all, that $R\left(\lambda ; L^{\prime}\right)$ 
can be thought of as defining a bounded linear transformation on $H_{0}$ to $\widehat{N}$ which we shall denote by $S_{\lambda}$; more precisely

$$
S_{\lambda} f^{0}=\hat{\gamma}\left[R\left(\lambda ; L^{\prime}\right) f^{0}\right] \text {. }
$$

Making use of the relation $\widehat{Q}\left(S_{\lambda} f^{0}, S_{\lambda} f^{0}\right)=\boldsymbol{Q}\left(\gamma R\left(\lambda ; L^{\prime}\right) f^{0}, \gamma R\left(\lambda ; L^{\prime}\right) f^{0}\right)$ and the identity $\left(\lambda I-L^{\prime}\right) R\left(\lambda ; L^{\prime}\right)=I$, we have

$$
\begin{aligned}
\widehat{Q}\left(S_{\lambda} f^{0}, S_{\lambda} f^{0}\right)= & \left\langle R\left(\lambda ; L^{\prime}\right) f^{0}, L^{\prime} R\left(\lambda ; L^{\prime}\right) f^{0}\right\rangle \\
& +\left\langle L^{\prime} R\left(\lambda ; L^{\prime}\right) f^{0}, R\left(\lambda ; L^{\prime}\right) f^{0}\right\rangle-\left\langle D R\left(\lambda ; L^{\prime}\right) f^{0}, R\left(\lambda ; L^{\prime}\right) f^{0}\right\rangle \\
= & 2 \lambda\left\langle R\left(\lambda ; L^{\prime}\right) f^{0}, R\left(\lambda ; L^{\prime}\right) f^{0}\right\rangle-\left\langle R\left(\lambda ; L^{\prime}\right) f^{0}, f^{0}\right\rangle \\
& -\left\langle f^{0}, R\left(\lambda ; L^{\prime}\right) f^{0}\right\rangle-\left\langle D R\left(\lambda ; L^{\prime}\right) f^{0}, R\left(\lambda ; L^{\prime}\right) f^{0}\right\rangle .
\end{aligned}
$$

If $L_{21}^{\prime}$ denotes the transformation with maximal negative graph which engenders $L^{\prime}$ and if $y^{1}=I_{01}^{-1} R\left(\lambda ; L^{\prime}\right) f^{0}$, then $\lambda I_{21} y^{1}-L_{21}^{\prime} y^{1}=I_{20} f^{0}$ and Lemma 1.3.2 implies that

$$
c(\lambda)^{2}\left|\left\langle D R\left(\lambda ; L^{\prime}\right) f^{0}, R\left(\lambda ; L^{\prime}\right) f^{0}\right\rangle\right| \leqq c(\lambda)^{2}\left\|y^{1}\right\|_{1}^{2}
$$

It readily follows that

$$
\leqq\left\|I_{20} f^{0}\right\|_{2}^{2} \leqq\left\|f^{0}\right\|^{2} \text {. }
$$

$$
\left\|S_{\lambda} f^{0}\right\|^{2}=-\widehat{Q}\left(S_{\lambda} f^{0}, S_{\lambda} f^{0}\right) \leqq k(\lambda)\left\|f^{0}\right\|^{2} .
$$

We now show that $k(\lambda) \leqq(2 \lambda)^{-1 / 2}$ by estimating the bound of the adjoint transformation $S_{\lambda}^{*}$ on $\widehat{N}$ to $H_{0}$. To this end, let $f^{0} \in H_{0}$ and $\widehat{\boldsymbol{y}} \in \widehat{\boldsymbol{N}}_{o}$ so that $\widehat{\boldsymbol{y}}=\hat{\gamma} y^{0}$ for some $y^{0} \in \mathfrak{D}\left(L^{\prime}\right)$. Then $\widehat{\boldsymbol{Q}}\left(S_{\lambda} f^{0}, \widehat{y}\right)=\boldsymbol{Q}\left(\gamma R\left(\lambda, L^{\prime}\right) f^{0}, \gamma y^{0}\right)$ and hence

$$
\widehat{\boldsymbol{Q}}\left(S_{\lambda} f^{0}, \widehat{\boldsymbol{y}}\right)=\left\langle R\left(\lambda ; L^{\prime}\right) f^{0}, L^{\prime} y^{0}\right\rangle+\left\langle L^{\prime} R\left(\lambda ; L^{\prime}\right) f^{0}, y^{0}\right\rangle-\left\langle D R\left(\lambda ; L^{\prime}\right) f^{0}, y^{0}\right\rangle .
$$

As the relation (2.3.14) shows $R_{10}\left(\lambda ; L^{\prime}\right) \equiv I_{01}^{-1} R\left(\lambda ; L^{\prime}\right)$ is a bounded linear transformation on $H_{0}$ to $H_{1}$. Hence we can rewrite the above equation as

$$
\widehat{\boldsymbol{Q}}\left(S_{\lambda} f^{0}, \widehat{y}\right)=\left\langle R_{10}\left(\lambda ; L^{\prime}\right) f^{0},\left(\lambda I_{21}-M_{21}^{1}\right) I_{01}^{-1} y^{0}\right\rangle-\left\langle f^{0}, y^{0}\right\rangle ;
$$

here we have again made use of the identity $\left(\lambda I-L^{\prime}\right) R\left(\lambda ; L^{\prime}\right)=I$. Next set $M^{\prime}=\left(L^{\prime}\right)^{*}$. Then $R^{*}\left(\lambda ; L^{\prime}\right)=R\left(\lambda ; M^{\prime}\right)$ so that if $z^{0} \in \mathfrak{D}\left(M^{\prime}\right)$ then

$$
\left\langle f^{0}, z^{0}\right\rangle=\left\langle R\left(\lambda ; L^{\prime}\right) f^{0}, \quad\left(\lambda I-M^{\prime}\right) z^{0}\right\rangle=\left\langle R_{10}\left(\lambda ; L^{\prime}\right) f^{0}, \quad\left(\lambda I_{21}-M_{21}^{1}\right) I_{01}^{-1} z^{0}\right\rangle .
$$

It is seen from the proof of Theorem 1.5.1 that $M^{\prime}$ is engendered from a transformation $M_{21}^{\prime}$ whose graph is a maximal negative subspace of $H_{12}$; in fact $L^{\prime}$ is similarly engendered from $L_{21}^{\prime}$ and $U\left[\mathfrak{S}\left(M_{21}^{\prime}\right)\right]$ is the $Q$-orthogonal complement to $\mathfrak{S}\left(L_{21}^{\prime}\right)$ and hence maximal positive. Moreover $S^{5}\left(I_{20} M^{\prime} I_{01}\right)$ is dense in $\mathscr{S}\left(M_{21}^{\prime}\right)$ and it follows from this and the above displayed relation that

$$
\left\langle f^{0}, I_{01} z^{1}\right\rangle=\left\langle R_{10}\left(\lambda ; L^{\prime}\right) f^{0},\left(\lambda I_{21}-M_{21}^{1}\right) z^{1}\right\rangle
$$


for all $z^{1} \in \mathfrak{D}\left(M_{21}^{\prime}\right)$. On the other hand, the $\Re\left(\lambda I_{21}-M_{21}^{\prime}\right)=H_{2}$ by Lemma 1.3.4. Hence there is a $z^{1} \in \mathfrak{D}\left(M_{21}^{\prime}\right)$ such that

$$
\left(\lambda I_{21}-M_{21}^{1}\right) I_{01}^{-1} y^{0}=\left(\lambda I_{21}-M_{21}^{1}\right) z^{1} .
$$

Combining this with (2.3.15) we obtain

$$
\left\langle S_{\lambda} f^{0}, \widehat{y}\right\rangle=-\widehat{\boldsymbol{Q}}\left(S_{\lambda} f^{0}, \widehat{\boldsymbol{y}}\right)=-\left\langle f^{0}, u^{0}\right\rangle,
$$

where $u^{0}=I_{01} z^{1}-y^{0}$. Setting $u^{1}=I_{01}^{-1} u^{0}$ we see that $M_{21}^{1} u^{1}=\lambda I_{21} u^{1} \in I_{20}\left[H_{0}\right]$, from which it follows that $u^{0} \in \mathfrak{D}\left(M_{1}\right)$ and hence that $M_{1} u^{0}=\lambda u^{0}$. Finally (2.3.16) shows that $S_{\lambda}^{*} \hat{y}=-u^{0}$.

Next we set $z^{0}=I_{01} z^{1}, \boldsymbol{y}=\gamma y^{0}, \boldsymbol{z}=\gamma z^{0}$, and $\boldsymbol{u}=\gamma u^{0}$. Then $y \in \beta\left[\left(\xi\left(L_{21}^{\prime}\right)\right]\right.$ and $z \in \beta\left[U\left[(\xi)\left(M_{21}^{\prime}\right)\right]\right]$. Consequently $y$ and $z$ are $\boldsymbol{Q}$-orthogonal and

$$
\begin{aligned}
& Q(u, u)=Q(y, y)+Q(z, z), \\
& Q(y, y) \leqq 0 \leqq Q(z, z) .
\end{aligned}
$$

Further

$$
\begin{aligned}
-Q(u, u) & =\left\langle M_{21}^{1} u^{1}, u^{1}\right\rangle+\left\langle u^{1}, M_{21}^{1} u^{1}\right\rangle-\left\langle D_{21} u^{1}, u^{1}\right\rangle \\
& =2 \lambda\left\langle I_{21} u^{1}, u^{1}\right\rangle-\left\langle D_{21} u^{1}, u^{1}\right\rangle \geqq 2 \lambda\left\langle u^{0}, u^{0}\right\rangle \geqq 0 .
\end{aligned}
$$

Combining (2.3.17) and (2.3.18) we obtain

$$
2 \lambda\left\langle u^{0}, u^{0}\right\rangle \leqq-Q(u, u) \leqq-Q(y, y)=-\hat{Q}(\hat{y}, \hat{y})=\langle\hat{y}, \hat{y}\rangle
$$

which shows that $2 \lambda\left\|S_{\lambda}^{*} \hat{y}\right\|^{2} \leqq\|\hat{y}\|^{2}$ for all $\hat{y} \in \widehat{N}_{o}$. Since $\widehat{N}_{o}$ is dense in $\widehat{N}$, this gives the required estimate on $\left\|S_{\lambda}^{*}\right\|$ and it follows that the same estimate holds for $\left\|S_{\lambda}\right\|=\left\|S_{\lambda}^{*}\right\|$. As a consequence $\left\|\hat{T} S_{\lambda}\right\|<1$ for $\lambda>\|\hat{T}\|^{2} / 2$ and it is easy to prove from this that $R(\lambda ; L)$ exists for such values of $\lambda$ (see, for instance, E. Hille and R. S. Phillips [7, Theorem 5.10.4]). In fact,

$$
R(\lambda ; L)=R\left(\lambda ; L^{\prime}\right) \sum_{n=0}^{\infty}\left[\hat{T} S_{\lambda}\right]^{n}, \quad \lambda>\|\hat{T}\|^{2} / 2,
$$

and for such $\lambda, \Re(\lambda I-L)=\mathfrak{D}[R(\lambda ; L)]=H_{0}$. This concludes the proof of Theorem 2.3.2.

\section{Corollary. $\Re\left[S_{\lambda}^{*}\right] \subset I_{01}\left[\mathfrak{I}\left(M_{21}^{1}\right)\right]$.}

Proof. In the course of the proof of the above theorem, it was shown for each $\hat{y} \in \widehat{N}_{o}$ that $u^{0}=S_{\lambda}^{*} \hat{y} \in \mathfrak{I}\left(M_{1}\right)$ and that $M_{1} u^{0}=\lambda u^{0}$. Combining (2.3.17) and (2.3.18), we see that

$$
0 \leqq-\left\langle D u^{0}, u^{0}\right\rangle \leqq-Q(u, u) \leqq-Q(y, y)=\langle\hat{y}, \hat{y}\rangle .
$$


Thus if $\left\{\hat{\boldsymbol{y}}_{n}\right\} \subset \widehat{\boldsymbol{N}}_{o}$ converges to $\widehat{\boldsymbol{y}} \in \widehat{\boldsymbol{N}}$, then $u_{n}^{0} \equiv S_{\lambda}^{*} \hat{y}_{n} \rightarrow u^{0} \equiv S_{\lambda}^{*} \hat{y}$ in $H_{0}$ and, by the above inequality,

$$
\left\langle D\left(u_{n}^{0}-u_{m}^{0}\right),\left(u_{n}^{0}-u_{m}^{0}\right)\right\rangle \rightarrow 0
$$

as $n, m \rightarrow \infty$. Setting $u_{n}^{1}=I_{01}^{-1} u_{n}^{0}$, it follows that $\left\{u_{n}^{1}\right\}$ converges in $H_{1}$, say to $u^{1}$, and that

$$
\left[u_{n}^{1}, M_{21}^{1} u_{n}^{1}\right]=\left[u_{n}^{1}, \lambda I_{21} u_{n}^{1}\right] \rightarrow\left[u^{1}, \lambda I_{21} u^{1}\right] \text { in } H_{12} .
$$

It is clear that $u^{0}=I_{01} u^{1}$ and, since $M_{21}^{1}$ is closed, we see that $u^{1} \in \mathfrak{D}\left(M_{21}^{1}\right)$.

We next characterize the adjoint $M$ of the maximal dissipative operator $L$ defined as in Theorem 2.3.2. According to Theorem 1.1.2, $M$ is again a maximal dissipative operator with dense domain. Further, $R(\lambda ; M)=R^{*}(\lambda ; L)$ and this together with (2.3.19) implies

(2.3.20) $\quad R(\lambda ; M)=R\left(\lambda ; M^{\prime}\right)+S_{\lambda}^{*} \hat{T}^{*}\left[\sum_{n=0}^{\infty}\left[S_{\lambda}^{*} \hat{T}^{*}\right]^{n}\right] R\left(\lambda ; M^{\prime}\right), \quad \lambda>\|\hat{T}\|^{2} / 2$,

where $M^{\prime}$ is the adjoint of $L^{\prime}$, defined as in the proof of Theorem 2.3.2. Since $M_{0} \subset M^{\prime} \subset M_{1}$, it follows from the corollary to Theorem 2.3.2 that

$$
\mathfrak{D}(M)=\Re[R(\lambda ; M)] \subset I_{01}\left[\mathfrak{D}\left(M_{21}^{1}\right)\right] .
$$

On the other hand, $L_{0} \subset L$ so that for $y^{0} \in \mathfrak{D}\left(L_{0}\right), z^{0} \in \mathfrak{D}(M), y^{1}=I_{01}^{-1} y^{0}$, and $z^{1}=I_{01}^{-1} z^{0}$ (well defined by $(2.3 .21)$ ), we have

$$
\left\langle I_{20} M z^{0}, y^{1}\right\rangle=\left\langle M z^{0}, y^{0}\right\rangle=\left\langle z^{0}, L_{0} y^{0}\right\rangle=\left\langle z^{1}, L_{21}^{0} y^{1}\right\rangle .
$$

Since $B\left(I_{20} L_{0} I_{01}\right)$ is dense in $\mathscr{S}\left(L_{21}^{0}\right)$, we conclude that $M_{21}^{1} z^{1}=I_{20} M z^{0}$ and hence that $M \subset M_{1}$.

Comparing the relations (2.3.2) and (2.3.7) we see that

$$
T=\hat{T} \hat{\gamma} \gamma^{-1} \text {. }
$$

Now for $y \in N$ we have

$$
\left\langle\hat{\gamma} \gamma^{-1} y, \hat{\gamma} \gamma^{-1} y\right\rangle=-\widehat{Q}\left(\hat{\gamma} \gamma^{-1} y, \hat{\gamma} \gamma^{-1} y\right)=-Q(y, y) \leqq 2\langle y, y\rangle .
$$

It follows that $\hat{\gamma} \gamma^{-1}$ is a bounded linear transformation on $N$ to $\widehat{N}$ and hence that $T$ is bounded on $N$ to $H_{0}$. Since $N \subset H$, the adjoint transformation $T^{*}$ can be thought of as a bounded transformation on $H_{0}$ to $H$, and for $y^{0} \in \mathfrak{D}(L)$ and $z^{0} \in H_{0}$

$$
\left\langle T \gamma y^{0}, z^{0}\right\rangle=\left\langle\gamma y^{0}, T^{*} z^{0}\right\rangle=Q\left(\gamma y^{0}, W T^{*} z^{0}\right)
$$

by (1.6.4). Finally for $y^{0} \in \mathfrak{D}(L)$ and $z^{0} \in \mathfrak{D}\left(M_{1}\right)$ 


$$
\begin{aligned}
\left\langle L y^{0}, z^{0}\right\rangle-\left\langle y^{0}, M_{1} z^{0}\right\rangle & =Q\left(\gamma y^{0}, \gamma z^{0}\right)+\left\langle T \gamma y^{0}, z^{0}\right\rangle \\
& =Q\left(\gamma y^{0}, \gamma z^{0}+W T^{*} z^{0}\right),
\end{aligned}
$$

and this vanishes for all $y^{0} \in \mathfrak{D}(L)$ if and only if $\gamma z^{0}+W T^{*} z^{0}$ belongs to the $Q$-orthogonal complement of $N$. We have therefore proved

Theorem 2.3.3. Let $L$ be defined as in Theorem 2.3.2 and set $M=L^{*}$. Then $M$ is a maximal dissipative restriction of $M_{1}$ with dense domain and

$$
\mathfrak{D}(M)=\left[z^{0} ; z^{0} \in \mathfrak{D}\left(M_{1}\right) \text { and } \gamma z^{0}+W T^{*} z^{0} \in P\right],
$$

where $\boldsymbol{P}$ is the $\mathbf{Q}$-orthogonal complement of $\boldsymbol{N}$.

The relation (2.3.23) shows that the presence of $T$ permits the rate at which energy enters the system through the boundary, namely $Q\left(U \gamma z^{0}, U \gamma z^{0}\right)$ $=-\boldsymbol{Q}\left(\gamma z^{0}, \gamma z^{0}\right)$, to be positive. However the inequality (2.3.10) together with the condition $\gamma z^{0}+W T^{*} z^{0} \in P$ require that the rate at which energy is dissipated in the interior of the system be at least as large as the flow in through the boundary.

2.4. An example. It will be instructive to carry through the previous development for the case of the telegraphist's equation

$$
u_{t t}+u_{t}-u_{x x}=0, \quad a<\chi<b, t>0 .
$$

This can be brought into the form of a system by setting

$$
\begin{aligned}
& \eta^{1}=u_{x}+u_{t}, \\
& \eta^{2}=u_{x}-u_{t},
\end{aligned}
$$

in which case

$$
\begin{aligned}
\eta_{t}^{1} & =\eta_{\chi}^{1}-2^{-1}\left(\eta^{1}-\eta^{2}\right), \\
\eta_{t}^{2} & =-\eta_{x}^{2}+2^{-1}\left(\eta^{1}-\eta^{2}\right) .
\end{aligned}
$$

In our usual notation with $y^{0}=\left[\eta^{1}, \eta^{2}\right]$,

$$
E=\left(\begin{array}{ll}
1 & 0 \\
0 & 1
\end{array}\right), \quad A=\left(\begin{array}{rr}
1 & 0 \\
0 & -1
\end{array}\right), \quad B=\left(\begin{array}{rr}
-1 / 2 & 1 / 2 \\
1 / 2 & -1 / 2
\end{array}\right), \quad D=\left(\begin{array}{rr}
-1 & 1 \\
1 & -1
\end{array}\right)
$$

this system is of the form (1.2). Since $I<F=I-D<3 I$, we see that the spaces $H_{0}, H_{1}$, and $H_{2}$ are essentially the same and in the following we shall not distinguish between them. The boundary integral takes the form

$$
\left.\left(A y^{0}, y^{0}\right)\right|_{a} ^{b}=\left.\left\{\left|\eta^{1}(\chi)\right|^{2}-\left|\eta^{2}(\chi)\right|^{2}\right\}\right|_{a} ^{b} .
$$

It can be shown (see [12]) that 


$$
\begin{aligned}
& \mathfrak{D}\left(L_{1}\right)=\left[y^{0} ; y^{0}(\chi) \text { absolutely continuous, } y^{0} \text { and } y_{x}^{0} \in L_{2}[(a, b) ; I]\right], \\
& \mathfrak{D}\left(L_{0}\right)=\left[y^{0} ; y^{0} \in \mathfrak{D}\left(L_{1}\right), y^{0}(a)=\theta=y^{0}(b)\right] .
\end{aligned}
$$

Moreover if $y^{0} \in \mathfrak{D}\left(L_{1}\right)$ and $a=-\infty[b=\infty]$, then of necessity

$$
y^{0}(a)=\theta\left[y^{0}(b)=\theta\right] .
$$

Thus for $(a, b)=(-\infty, \infty), \mathfrak{D}\left(L_{0}\right)=\mathfrak{D}\left(L_{1}\right)$, the boundary space $H$ consists of only the zero element, and $L=L_{0}=L_{1}$ is the only maximal dissipative extension of $L_{0}$ as well as the only maximal dissipative restriction of $L_{1}$.

When $(a, b)=(0, \infty)$ the problem becomes more interesting. The boundary space $H$ is now two dimensional and $y=\beta\left[y^{0}, L_{1} y^{0}\right]$ can be represented componentwise by $\left[\eta^{1}(0), \eta^{2}(0)\right]$, in which case

$$
\boldsymbol{Q}(\boldsymbol{y}, \boldsymbol{z})=\eta^{2}(0)\left[\zeta^{2}(0)\right]^{-}-\eta^{1}(0)\left[\zeta^{1}(0)\right]^{-} .
$$

It is readily seen that each maximum negative subspace $N$ of $H$ is determined by an $\alpha$ of absolute value $\leqq 1$ and is of the form

$$
N=\left[\left[\eta^{1}(0), \eta^{2}(0)\right] ; \eta^{2}(0)=\alpha \eta^{1}(0)\right] .
$$

The hilbert space $\widehat{N}$ is one dimensional unless $|\alpha|=1$, in which case it consists of only the zero element. Representing $\hat{\gamma} y^{0}$ by $\eta^{1}(0)$ we see that

$$
\widehat{Q}\left(\hat{\gamma} y^{0}, \hat{\gamma} z^{0}\right)=\left(|\alpha|^{2}-1\right) \eta^{1}(0)\left[\zeta^{1}(0)\right]^{-} .
$$

It is clear that the most general form of $\hat{T}$ is given by

$$
\hat{T} \hat{\gamma} y^{0}=c \eta^{1}(0) \text { where } \quad c=\left(\begin{array}{l}
c_{1}(\chi) \\
c_{2}(\chi)
\end{array}\right)
$$

and $c_{i} \in L_{2}(0, \infty)$. Condition (2.3.10) becomes

$$
\left(1-|\alpha|^{2}\right)^{-1}\left|\left\langle c, y^{0}\right\rangle\right|^{2} \leqq-\left\langle D y^{0}, y^{0}\right\rangle,
$$$$
y^{0} \in H_{0} .
$$

In order to see what restriction this imposes on $c$ we diagonalize $D$, say by the rotation $U$, and obtain

$$
U^{*} D U=\left(\begin{array}{rr}
0 & 0 \\
0 & -2
\end{array}\right) \text { where } U=\left(u_{j k}\right)=2^{-1 / 2}\left(\begin{array}{rr}
1 & -1 \\
1 & 1
\end{array}\right) .
$$

Setting $w^{0}=U^{*} y^{0}$, the relation (2.4.9) takes the form

$$
\left|\left\langle d_{1}, \omega^{1}\right\rangle+\left\langle d_{2}, \omega^{2}\right\rangle\right|^{2} \leqq 2\left(1-|\alpha|^{2}\right)\left\langle\omega^{2}, \omega^{2}\right\rangle
$$

where $d_{k}=\sum_{j} \bar{u}_{j k} c_{j}, k=1,2$. It follows that (2.3.10) is equivalent with $d_{1}=0$ and $\left\langle d_{2}, d_{2}\right\rangle \leqq 2\left(1-|\alpha|^{2}\right)$ and hence with

$$
c_{1}=-c_{2} \text { and }\left\langle c_{1}, c_{1}\right\rangle \leqq 1-|\alpha|^{2} .
$$

According to Theorem 2.3.2, the operator 


$$
\begin{aligned}
L y^{0} & =L_{1} y^{0}+c \eta^{1}(0), \\
\mathfrak{D}(L) & =\left[y^{0} ; y^{0} \in D\left(L_{1}\right), \eta^{2}(0)=\alpha \eta^{1}(0)\right]
\end{aligned}
$$

where $c=\left[c_{1},-c_{1}\right]$ and $\left\langle c_{1}, c_{1}\right\rangle \leqq 1-|\alpha|^{2} \geqq 0$, defines a maximal dissipative extension of $L_{0}$. The adjoint operator $M=L^{*}$ is described in Theorem 2.3.3. Thus $M$ is a restriction of

$$
M_{1} z^{0}=-\left(A z^{0}\right)_{\chi}+B^{*} z^{0}, \mathfrak{D}\left(M_{1}\right)=\mathfrak{I}\left(L_{1}\right),
$$

in fact the domain of $M$ is readily found from the relation

$$
\begin{aligned}
\left\langle L y^{0}, z^{0}\right\rangle-\left\langle y^{0}, M_{1} z^{0}\right\rangle & =\eta^{2}(0)\left[\zeta^{2}(0)\right]^{-}-\eta^{1}(0)\left[\zeta^{1}(0)\right]^{-}+\left\langle c, z^{0}\right\rangle \eta^{1}(0) \\
& =\eta^{1}(0)\left[\alpha\left[\zeta^{2}(0)\right]^{-}-\left[\zeta^{1}(0)\right]^{-}+\left\langle c, z^{0}\right\rangle\right]
\end{aligned}
$$

and this vanishes for all $y^{0} \in \mathfrak{D}(L)$ (and hence for all $\eta^{1}(0)$ ) if and only if

$$
\left\langle z^{0}, c\right\rangle \equiv\left\langle\left(\zeta^{1}-\zeta^{2}\right), c_{1}\right\rangle=\zeta^{1}(0)-\bar{\alpha} \zeta^{2}(0) .
$$

The global lateral Condition (2.4.13) serves to define $\mathfrak{D}(M) \subset \mathfrak{I}\left(M_{1}\right)$ and assuming $\left\langle c_{1}, c_{1}\right\rangle \leqq 1-|\alpha|^{2} \geqq 0, M$ is a maximal dissipative restriction of $M_{1}$ by Theorem 2.3.3.

The case $-\infty<a<b<\infty$ is slightly more complex. Here the boundary space $H$ is four dimensional and $y=\beta\left[y^{0}, L_{1} y^{0}\right]$ can be represented componentwise by $\left[\eta^{1}(b), \eta^{2}(b), \eta^{1}(a), \eta^{2}(a)\right]$, in which case

(2.4.14) $\boldsymbol{Q}(\boldsymbol{y}, \boldsymbol{z})=\eta^{1}(b)\left[\zeta^{1}(b)\right]^{-}-\eta^{2}(b)\left[\zeta^{2}(b)\right]^{-}-\eta^{1}(a)\left[\zeta^{1}(a)\right]^{-}+\eta^{2}(a)\left[\zeta^{2}(a)\right]^{-}$.

Each maximal negative subspace $N$ of $H$ is now two dimensional and can be characterized by four constants $\delta, \epsilon, \mu, \nu$ as

$$
\begin{aligned}
& \eta^{1}(b)=\delta \eta^{2}(b)+\epsilon \eta^{1}(a), \\
& \eta^{2}(a)=\mu \eta^{2}(b)+\nu \eta^{1}(a),
\end{aligned}
$$

provided

$$
\left|\delta \eta^{2}(b)+\epsilon \eta^{1}(a)\right|^{2}+\left|\mu \eta^{2}(b)+\nu \eta^{1}(a)\right|^{2}-\left|\eta^{2}(b)\right|^{2}-\left|\eta^{1}(a)\right|^{2}
$$

is a negative quadratic form in $\eta^{2}(b), \eta^{1}(a)$. Necessary and sufficient conditions for this are

$$
\begin{gathered}
|\delta|^{2}+|\mu|^{2} \leqq 1 \\
|\delta|^{2}+|\epsilon|^{2}+|\mu|^{2}+|\nu|^{2} \leqq 1+|\delta \nu-\epsilon \mu|^{2} .
\end{gathered}
$$

Replacing the $\leqq$ signs by $<$ signs, the Conditions (2.4.17) become necessary and sufficient for the form to be negative definite. It is convenient at this point to diagonalize the form $(2.4 .16)$ by a rotation of coordinates: $\left[\eta_{i}^{1}, \eta_{i}^{2}\right]$ $=V\left[\eta^{2}(b), \eta^{1}(a)\right]$, in terms of which we have for $\gamma y^{0}, \gamma z^{0} \in N$

$$
\boldsymbol{Q}\left(\gamma y^{0}, \gamma z^{0}\right)=-\left[q_{1} \eta_{i}^{1}\left[\zeta_{i}^{1}\right]^{-}+q_{2} \eta_{i}^{2}\left[\xi_{i}^{2}\right]^{-}\right] .
$$

If $N$ is (maximal) negative, then $q_{j} \geqq 0, j=1,2$. The hilbert space $\widehat{N}$ will now 
be two, one, or zero dimensional according as the $q_{j}$ 's are both positive, only one is positive, or both are zero. If both $q_{j}^{\prime}$ 's are positive we may write $\hat{\gamma} y^{0}$ $=\gamma y^{0}$ and define

$$
\hat{T} \hat{\gamma} y^{0}=C\left(\begin{array}{c}
1 \\
\eta_{i} \\
2 \\
\eta_{i}
\end{array}\right) \text {, where } C=\left(c_{k j}(\chi)\right) .
$$

Actually this formalism creates no difficulty even if say $q_{j}=0$ provided we then set $c_{k j}=0$ for $k=1,2$. Condition (2.3.10) now becomes

$$
\sum_{j=1}^{2} q_{j}^{-1}\left|\sum_{k=1}^{2}\left\langle\eta^{k}, c_{k j}\right\rangle\right|^{2} \leqq-\left\langle D y^{0}, y^{0}\right\rangle
$$

Again, diagonalizing $D$ with $w^{0}=U^{*} y^{0}$, the Condition (2.4.20) becomes

$$
\sum_{j=1}^{2} q_{j}^{-1}\left|\sum_{k, l=1}^{2}\left\langle u_{k l} \omega^{l}, c_{k j}\right\rangle\right|^{2} \leqq 2\left\langle\omega^{2}, \omega^{2}\right\rangle .
$$

For $\omega^{2}=0$ we see that this implies $\sum_{k} u_{k 1} c_{k j} \equiv 2^{-1 / 2}\left(c_{1 j}+c_{2 j}\right)=0$, providing that $q_{j} \neq 0$. Hence setting $c_{1}=-c_{11}, c_{2}=c_{22},(2.4 .21)$ becomes

$$
q_{1}^{-1}\left|\left\langle\omega^{2}, c_{1}\right\rangle\right|^{2}+q_{2}^{-1}\left|\left\langle\omega^{2}, c_{2}\right\rangle\right|^{2} \leqq\left\langle\omega^{2}, \omega^{2}\right\rangle .
$$

If, say, $q_{1}=0 \neq q_{2}$, then as above $c_{1}=0$ and the Condition (2.3.10) becomes

$$
\left\langle c_{2}, c_{2}\right\rangle \leqq q_{2} .
$$

On the other hand if both $q_{i}$ 's are positive, then Condition (2.3.10) is equivalent with

$$
d_{11}+d_{22}+\left[\left(d_{11}+d_{22}\right)^{2}-4 d\right]^{1 / 2} \leqq 2,
$$

where $d_{i j}=\left(q_{i} q_{j}\right)^{-1 / 2}\left\langle c_{1}, c_{j}\right\rangle$ and $d=d_{11} d_{22}-\left|d_{12}\right|^{2}$. Assuming $c_{1}$ and $c_{2}$ to be so restricted, assuming $N$ to be a maximal negative subspace defined as above, and assuming $\hat{T}$ to be given by (2.4.19), then the operator $L$ defined as in Theorem 2.3.2 is a maximal dissipative extension of $L_{0}$.

The adjoint operator $M=L^{*}$ is again maximal dissipative and a restriction of $M_{1}$. Moreover $\mathfrak{D}(M)$ is delimited by global lateral conditions and again these conditions are readily obtained from the relation

$$
\begin{aligned}
\left\langle L y^{0}, z^{0}\right\rangle-\left\langle y^{0}, M_{1} z^{0}\right\rangle= & \left(\delta \eta^{2}(b)+\epsilon \eta^{1}(a)\right)\left[\zeta^{1}(b)\right]--\eta^{2}(b)\left[\zeta^{2}(b)\right]^{-} \\
& -\eta^{1}(a)\left[\zeta^{1}(a)\right]^{-}+\left(\mu \eta^{2}(b)+\nu \eta^{1}(a)\right)\left[\zeta^{2}(a)\right]^{-} \\
& +\eta^{2}(b)\left[\left\langle c_{11}^{\prime}, \zeta^{1}\right\rangle+\left\langle c_{21}^{\prime}, \zeta^{2}\right\rangle\right] \\
& +\eta^{1}(a)\left[\left\langle c_{12}^{\prime}, \zeta^{1}\right\rangle+\left\langle c_{22}^{\prime}, \zeta^{2}\right\rangle\right],
\end{aligned}
$$

where $\left(c_{i j}^{\prime}\right)=C V$. The left member vanishes for all $y^{0} \in \mathfrak{D}(L)$ (and hence for all $\left.\left[\eta^{2}(b), \eta^{1}(a)\right]\right)$ if and only if 


$$
\begin{aligned}
& \left\langle\zeta^{1}, c_{11}^{\prime}\right\rangle+\left\langle\zeta^{2}, c_{21}^{\prime}\right\rangle=\zeta^{2}(b)-\bar{\delta} \zeta^{1}(b)-\bar{\mu} \zeta^{2}(a), \\
& \left\langle\zeta^{1}, c_{12}^{\prime}\right\rangle+\left\langle\zeta^{2}, c_{22}^{\prime}\right\rangle=\zeta^{1}(a)-\bar{\epsilon} \zeta^{1}(b)-\bar{\nu} \zeta^{2}(a) .
\end{aligned}
$$

These are the required lateral conditions determining $\mathfrak{D}(M) \subset \mathfrak{D}\left(M_{1}\right)$. We recall that $c_{j}=0$ if $q_{j}=0$ and hence when both $q_{j}$ 's vanish, then $C=\Theta$ and the left members of (2.4.24) are each zero so that the lateral conditions become boundary conditions; in this case both $L$ and $M$ are properly dissipative operators.

\section{REFERENCES}

1. J. W. Calkin, Abstract symmetric boundary conditions, Trans. Amer. Math. Soc. vol. 45 (1939) pp. 369-442.

2. J. L. B. Cooper, One parameter semi-groups of isometric operators in Hilbert space, Ann. of Math. vol. 48 (2) (1947) pp. 827-842.

3. - Symmetric operators in Hilbert space, Proc. London Math. Soc. vol. 50 (2) (1948) pp. 11-15.

4. H. Federer, Measure and area, Bull. Amer. Math. Soc. vol. 58 (1952) pp. 306-378.

5. W. Feller, The parabolic differential equations and the associated semi-groups of transformations, Ann. of Math. vol. 55 (1952) pp. 468-519.

6. K. O. Friedrichs, Symmetric hyperbolic linear differential equations, Comm. Pure Appl. Math. vol. 7 (1954) pp. 345-392.

7. E. Hille and R. S. Phillips, Functional analysis and semi-groups, Amer. Math. Soc. Colloquium Publications, vol. 31, 1957.

8. Lars Hörmander, General partial differential operators, Acta Math. vol. 94 (1955) pp. $161-248$.

9. M. S. Livšic, On the spectral decomposition of linear nonself-adjoint operators, Mat. Sbornik, N. S. vol. 34 (76) (1954) pp. 145-199.

10. B. R. Mukminov, On expansions with respect to the eigenfunctions of dissipative kernels, Doklady Akad. Nauk. SSSR, N. S. vol. 99 (1954) pp. 499-502.

11. B. de Sz. Nagy, Spektraldarstellung linearer Transformationen des Hilbertschen Raumes, Ergebnisse d. Math. vol. 5 (1942).

12. R. S. Phillips, Dissipative hyperbolic systems, Trans. Amer. Math. Soc. vol. 86 (1957) pp. 109-173.

13. M. I. Višik, On general boundary problems for elliptic differential equations, Trudy Moskov. Mat. Obšc. vol. 1 (1952) pp. 187-246.

14. Kôsaku Yosida, An operator-theoretical integration of the wave equation, J. Math. Soc. Japan vol. 8 (1956) pp. 79-92.

The University of Southern California $\left({ }^{\circ}\right)$, Los ANgeles, Calif.

(9) Now at the University of California at Los Angeles. 